\title{
The morbid anatomy of the human genome: chromosomal location of mutations causing disease
}

\author{
Victor A McKusick, Joanna S Amberger
}

\begin{abstract}
Information is given in tabular form derived from a synopsis of the human gene map which has been updated continuously since 1973 as part of Mendelian Inheritance in Man (Johns Hopkins University Press, 10th ed, 1992) and of OMIM (Online Mendelian Inheritance in Man, available generally since 1987). The part of the synopsis reproduced here consists of chromosome by chromosome gene lists of loci for which there are associated disorders (table 1), a pictorial representation of this information (fig 1a-d), and an index of disorders for which the causative mutations have been mapped (table 2).

In table 1, information on genes that have been located to specific chromosomal positions and are also the site of disease producing mutations is arranged by chromosome, starting with chromosome 1 and with the end of the short arm of the chromosome in each case.

In table 2 an alphabetised list of these disorders and the chromosomal location of the mutation in each case are provided. Both in the 'Disorder' field of table 1 and in table 2, the numbers 1,2 , or 3 in parentheses after the name of the disorder indicate that its chromosomal location was determined by mapping of the wildtype gene (1), by mapping of the clinical phenotype (2), or by both strategies (3).

(f Med Genet 1993;30:1-26)
\end{abstract}

The fields in the listing by chromosome (table 1) are as follows:

Location - that is, chromosomal site $(\mathrm{p}=$ short arm; $\mathrm{q}=$ long arm; numbers $=$ band; ter $=$ end).

Symbol - The symbol approved by the Nomenclature Committee of the Human Gene Mapping Workshops and their successor organisation is given first; alternative symbols are included.

Status - $\mathrm{C}=$ confirmed; $\quad \mathbf{P}=$ provisional; $\mathbf{L}=$ 'in limbo' (that is, tentative or inconsistent). The 'in limbo' entries are not listed in fig 1 .
Title - name of gene locus.

MIM\# - This is the number in McKusick's Mendelian Inheritance in Man, 10th ed (1992) and its continuously updated online version OMIM. (For historical reasons, the number may sometimes indicate location of the entry in the 'dominant catalogue' because the wildtype gene was characterised and mapped before the recessive disorder resulting from mutation at that site. The practice is to create only one entry in OMIM for each gene locus.)

\section{Method of mapping}

A = in situ DNA-RNA or DNA-DNA annealing ('hybridisation'), for example, ribosomal RNA genes to acrocentric chromosomes; kappa light chain genes to chromosome 2 .

AAS = deductions from the amino acid sequence of proteins, for example, linkage of delta and beta globin loci from study of hemoglobin Lepore.

$\mathbf{C}=$ chromosome mediated gene transfer (CMGT), for example, cotransfer of galactokinase and thymidine kinase.

Ch $=$ chromosomal change associated with particular phenotype and not proved to represent linkage (Fc), deletion (D), or virus effect (V), for example, loss of 13q14 band in some cases of retinoblastoma.

$\mathbf{D}=$ deletion or dosage mapping (concurrence of chromosomal deletion and phenotypic evidence of hemizygosity), trisomy mapping (presence of three alleles in the case of a highly polymorphic locus), or gene dosage effects (correlation of trisomic state of part or all of a chromosome with $50 \%$ more gene product). Includes 'loss of heterozygosity' (loss of alleles) in malignancies. Examples: acid phosphatase-1 to chromosome 2; glutathione reductase to chromosome 8 . Includes DNA dosage, for example, fibrinogen loci to $4 \mathrm{q} 2$. Dosage mapping also includes coamplification in tumour cells.

$\mathbf{E M}=$ exclusion mapping, that is, narrowing the possible location of loci by exclusion of parts of the map by deletion mapping, extended to include negative lod scores from families with marker chromosomes and negative lod scores with other assigned loci, for example, support for assignment of MNSs to $4 \mathrm{q}$. 
$\mathbf{F}=$ linkage study in families, for example, linkage of ABO blood group and nail-patella syndrome. (When a chromosomal heteromorphism or rearrangement is one trait, Fc is used, for example, Duffy blood group locus on chromosome 1 . When one or both of the linked loci are identified by a DNA polymorphism, $\mathrm{Fd}$ is used, for example, Huntington's disease on chromosome 4. F=L in the HGM workshops.)

$\mathbf{H}=$ based on presumed homology, for example, proposed assignment of $\mathrm{TF}$ to $3 \mathrm{q}$. Mainly heuristic or confirmatory.

HS $=$ DNA/cDNA molecular hybridisation in solution ('Cot analysis').

$\mathbf{L}=$ lyonisation, for example, OTC to $\mathrm{X}$ chromosome. ( $\mathrm{L}=$ symbol for family linkage study in the HGM workshops.)

$\mathbf{L D}=$ linkage disequilibrium, for example, beta and delta globin genes.

$\mathbf{M}=$ Microcell mediated gene transfer (MMGT), for example, a collagen gene (COL1A1) to chromosome 17.

OT = ovarian teratoma (centromere mapping), for example, PGM3 and centromere of chromosome 6.

$\mathbf{P c m}=$ PCR of microdissected chromosome segments (see REl).

Psh $=$ PCR of somatic cell hybrid DNA.

$\mathbf{R}=$ radiation hybrids, that is, irradiation of cells followed by 'rescue' through fusion with non-irradiated (non-human) cells (Goss-Harris method of radiation-induced gene segregation), for example, order of genes on Xq.

$\mathbf{R E}=$ restriction endonuclease techniques, for example, fine structure map of the beta globin cluster (HBBC) on $11 \mathrm{p}$; physical linkage of 3 fibrinogen genes (on 4q).

REa = combined with somatic cell hybridisation, for example, NAG (HBBC) to $11 \mathrm{p}$.

$\mathbf{R E b}=$ combined with chromosome sorting, for example, insulin to $11 \mathrm{p}$. Includes Lebo's adaptation (dual laser chromosome sorting and spot blot DNA analysis), for example, MGP to 11q. (For this method, using flow sorted chromosomes, W was the symbol adopted by the HGM workshops.)

$\mathbf{R E c}=$ hybridisation of $\mathrm{cDNA}$ to genomic fragment (by YAC, PFGE, microdissection, etc), for example, A-11 on Xq.

REl = isolation of gene from chromosome specific genomic library (see Pcm).

$\mathbf{R E n}=$ neighbour analysis in restriction fragments, for example, in pulsed field gel electrophoresis (PFGE).

$\mathbf{S}=$ 'segregation' (cosegregation) of human cellular traits and human chromosomes (or segments of chromosomes) in particular clones from interspecies somatic cell hybrids, for example, thymidine kinase to chromosome 17. When with restriction enzyme, REa; with hybridisation in solution, HS.

$\mathbf{V}=$ induction of microscopically evident chromosomal change by a virus, for example, adenovirus 12 changes on chromosomes 1 and 17 .
$\mathbf{X} / \mathbf{A}=\mathbf{X}$-autosome translocation in female with $\mathrm{X}$ linked recessive disorder, for example, assignment of Duchenne muscular dystrophy to $\mathrm{Xp} 21$.

Disorder - Allelic disorders are separated by semicolons. Brackets [] indicate a 'nondisease', that is, a variation with no definite adverse consequences. Braces \{\} indicate specific susceptibility or resistance with monogenic basis. $(1)=$ wildtype gene mapped. (2) = disease phenotype mapped. (3)= both wildtype gene and disease phenotype mapped.

Mouse - Mouse chromosome carrying homologous gene.

References for the mapping information and additional information about the loci and disorders are provided in Mendelian Inheritance in Man, 10th ed (McKusick, 1992) and $\mathrm{OMIM}^{\mathrm{TM}}$ (Online Mendelian Inheritance in Man). To obtain information on accessing OMIM, contact:

In the United States: GDB/OMIM User Support, Welch Medical Library, 1830 E Monument Street, 3rd Floor, Baltimore, MD 21205, USA (Telephone: 410-955-7058, Fax: 410-955-0054, e-mail: help@,welch.jhu.edu).

In the United Kingdom: Christine Bates, Human Gene Mapping Program Resource Centre, CRC, Watford Road, Harrow, Middx HA1 3UJ, UK (Telephone: 44-81-869-3446, Fax: 44-81-869-3807, e-mail: cbates@uk.ac.crc).

In Germany: Otto Ritter, German Cancer Research Centre (DKFZ), Molecular Biophysics Group, Im Neuenheimer Feld 280, D-6900 Heidelberg 1, FRG (Telephone: 49-6221-42-2372, Fax: 49-6221-40-1271, e-mail: dok261@cvx12.dkfz-heidelberg.de).

In Australia: Alex Reisner, ANGIS, Electrical Engineering Building, JO3, University of Sydney, Sydney, NSW 2006, Australia, (Telephone: 61-2-692-294, Fax 61-2-692-3847, e-mail: reisner@ee.su.oz.au).

\section{Discussion}

In these listings, 738 disorders in total are indicated as assigned to specific chromosomes and, in most instances, to specific regions of those chromosomes. (More than 50 other disorders are known to be caused by mutations in genes on the $\mathrm{X}$ chromosome but their regional location is not known and therefore they have not been included here.) The 738 disorders are distributed over 625 loci; many loci have more than one allelic mutation producing distinct phenotypes.

Some of the disorders (labelled with the number 1) have been mapped to specific sites by virtue of mapping of the wildtype gene; many forms of chronic non-spherocytic haemolytic anaemia such as those due to pyruvate kinase deficiency, triosephosphate isomerase deficiency, and bisphosphoglycerate mutase deficiency are examples.

Other disorders (labelled with the number 2) are mapped only on the basis of the clinical phenotype which is found to be linked to markers at a particular chromosomal site or the phenotype is found in association with a 
chromosomal aberration. Huntington's disease is an example of a disorder mapped by linkage to DNA markers.

Yet other disorders (labelled with the number 3) have been mapped by both approaches. The form of elliptocytosis due to a defect in the gene encoding protein 4.1 is an early example, and piebaldism (due to mutation in the KIT gene) is a recent one.

Chromosomal aberrations have contributed to the mapping of Mendelian disorders to an extent that perhaps would not have been predicted. Both translocations and deletions have been informative. Because of the extensive homology of synteny shown between mouse and man, comparative mapping has also had great heuristic and confirmatory value in identifying the location of genes and genetic disorders in the human.

Mapping of genetic disorders has shed light on both allelism and non-allelism. Both phenomena are illustrated by the haemoglobinopathies and the collagenopathies. Because of the heteromeric structure of the haemoglobin molecule, thalassaemia, Heinz body haemolytic anaemia, erythraemia, or methaemoglobinaemia can result from mutation in either the $\alpha$ globin gene on chromosome 16 or the $\beta$ globin gene on chromosome 11. Similarly, in the case of the type I collagen molecule, which is also heteromeric, two or more different forms of osteogenesis imperfecta, as well as the distinct disorder Ehlers-Danlos syndrome, can occur from various mutation in either the gene for the $\alpha 1$ chain (COL1A1) on chromosome 17 or that of the $\alpha 2$ chain (COL1A2) on chromosome 7 .

Allelic series as the basis of phenotypic diversity are well illustrated by Duchenne and Becker muscular dystrophies and by the several forms of spinal muscular atrophy. Gardner syndrome and adenomatous polyposis coli (without extra-bowel manifestations) are demonstrably allelic; indeed the fact that the same mutation has been shown to cause one or the other of these two disorders in different members of the same family indicates that the two syndromes represent variability of expression.

Mapping information suggests that some syndromes with a combination of manifestations may result from mutation in a complex locus, that is, the particular pattern of components may be determined by the part of the gene affected by the mutation. Von HippelLindau syndrome and multiple endocrine neoplasia type II are possible examples. Close linkage of mutant genes, each responsible for a specific component, in general is rejected as the cause of genetic syndromes, in favour of pleiotropism. The bona fide nature of some so called contiguous gene syndromes is unquestionable, however.

Somatic cell genetic disease is particularly well illustrated by many forms of neoplasia, both solid tumours and leukaemias, that have been traced to genes located at specific sites. Many are listed among the disorders here. Some congenital malformations also represent somatic cell genetic disorders. The Happle hypothesis holds that some disorders are due to the mosaic state of mutations which would be lethal if present in the non-mosaic state, even in the heterozygote. The McCune-Albright syndrome (Albright polyostotic fibrous dysplasia), pseudohypoparathyroidism (Albright hereditary osteodystrophy), and growth hormone secreting pituitary tumour comprise a remarkable set of genetic diseases illustrating an allelic series. The first, a somatic cell genetic disease, is due to an activating mutation in the GNAS1 gene occurring in early embryogenesis; the second is a loss of function mutation of the GNAS1 gene, and the third represents an activating somatic mutation occurring in a single cell of the anterior pituitary.

We are indebted to colleagues who have participated in the Human Gene Mapping Workshops 1973 to 1991 and assisted in the collation of data on the human gene map. The development and maintenance of Mendelian Inheritance in Man and its online version OMIM was supported in part by the Howard Hughes Medical Institute from 1986 to 1991. It is now supported, in the main, jointly by the NIH and the DOE as part of the Genome Data Base (GDB), the repository for mapping information coming from the Human Genome Project.

1 McKusick VA: Mendelian inheritance in man, 10th ed. Baltimore: The Johns Hopkins University Press, 1992.

Editorial note. It is our intention to publish an annual update of this information. 

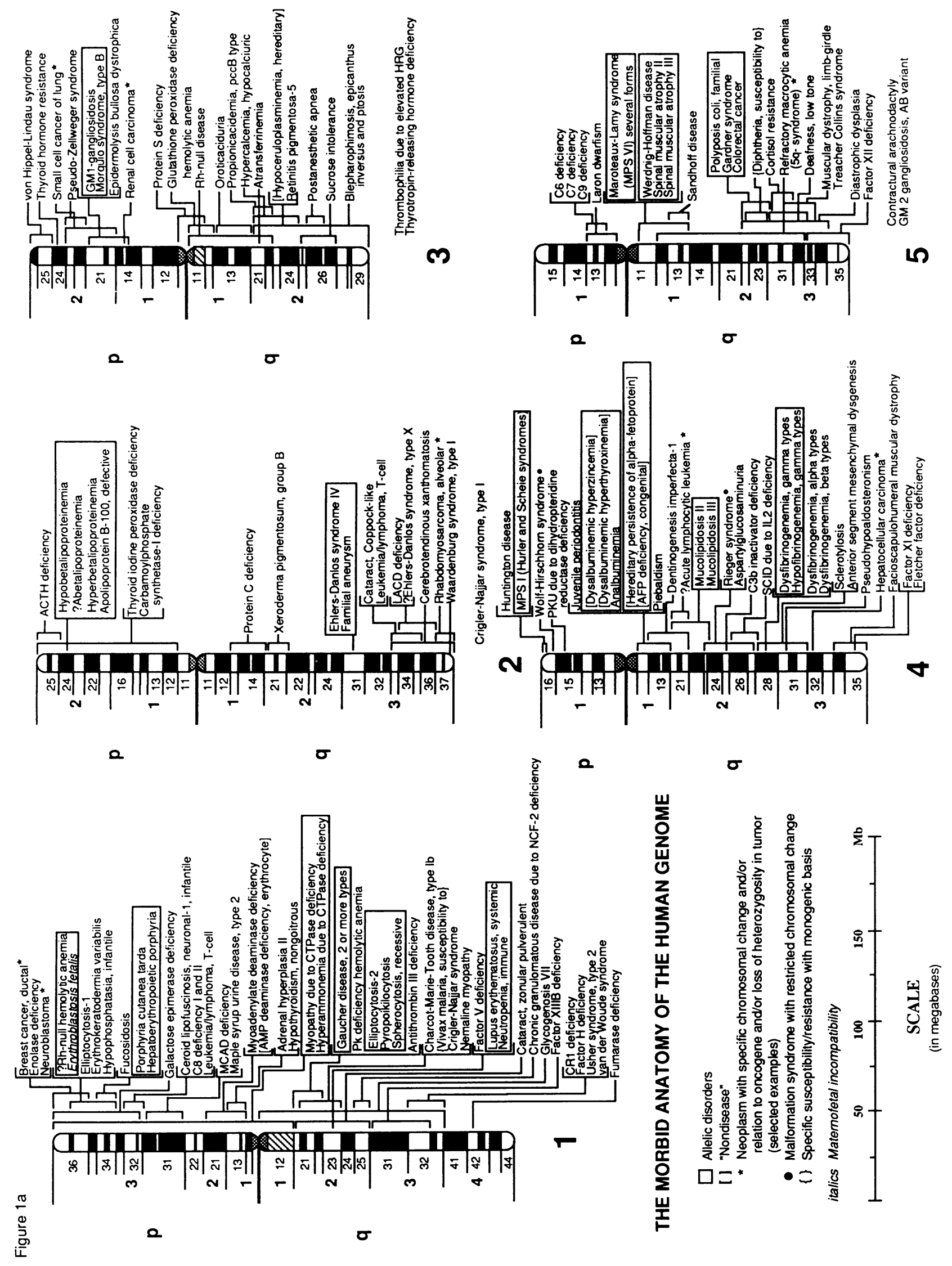

Figure 1a-d The morbid anatomy of the human genome: disorders with confirmed or provisional assignments have been included. Because of the large number of disorders assigned to specific regions of the $X$ chromosome, only selected ones are represented here. 

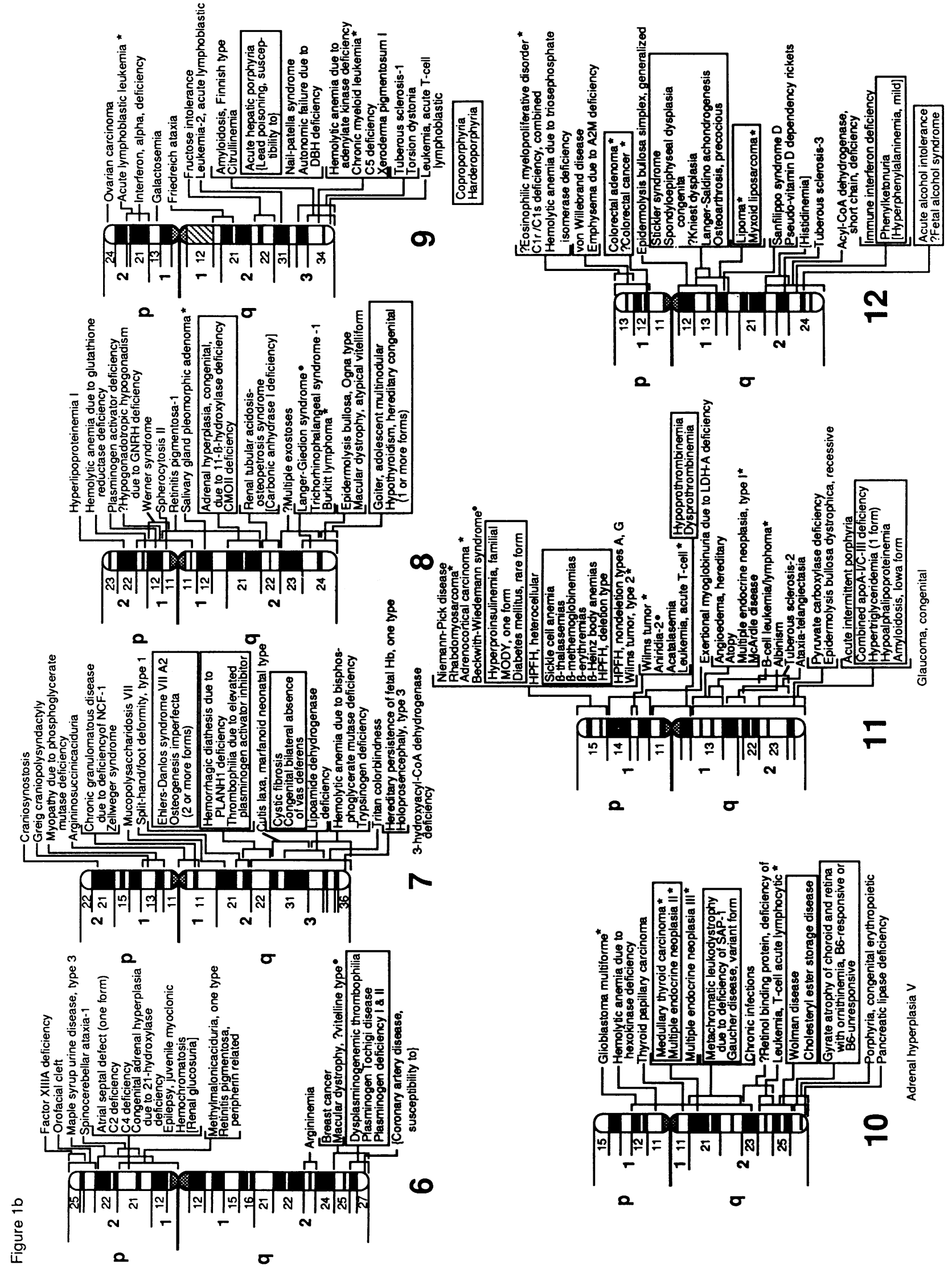

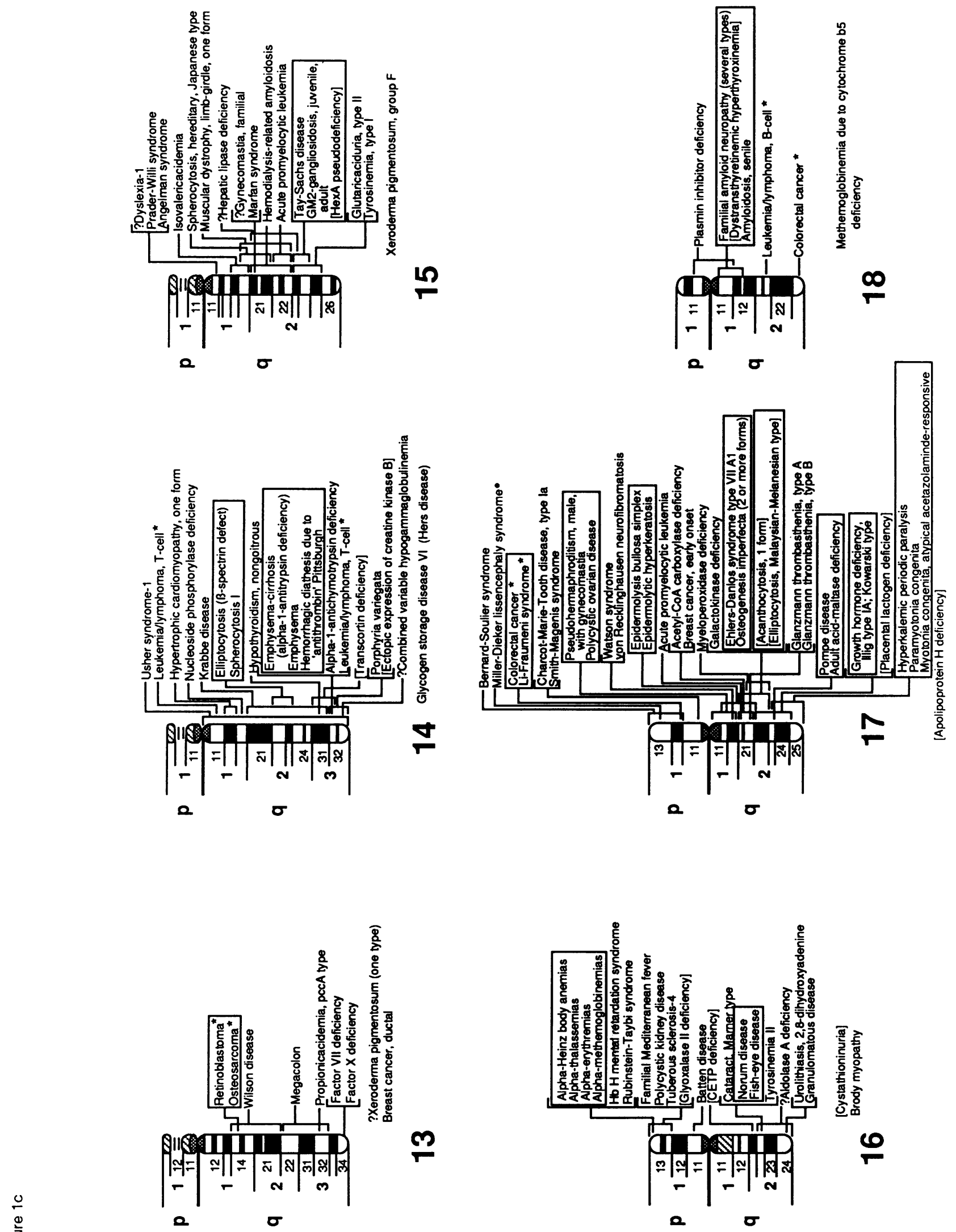

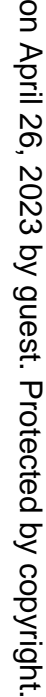



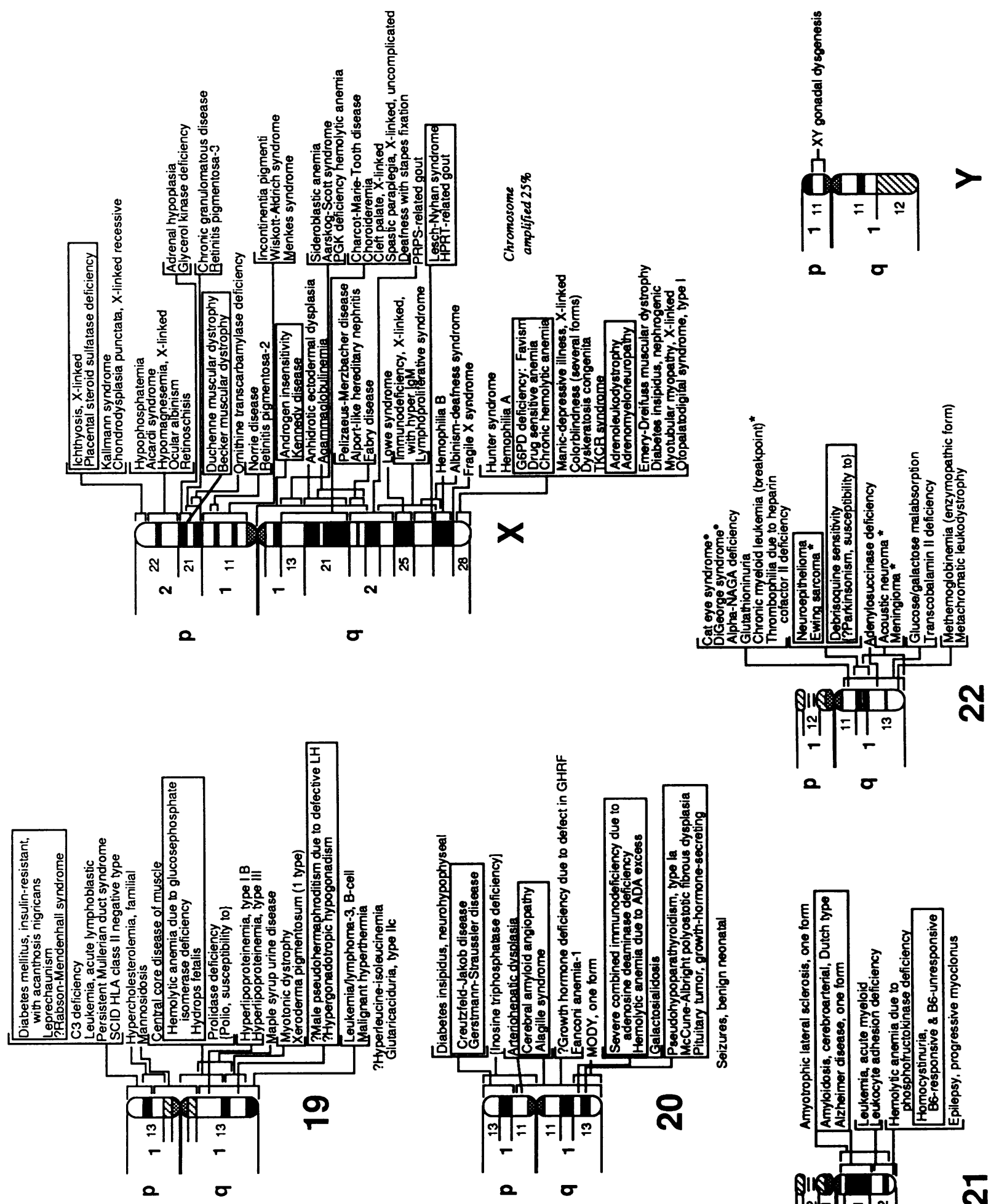

음

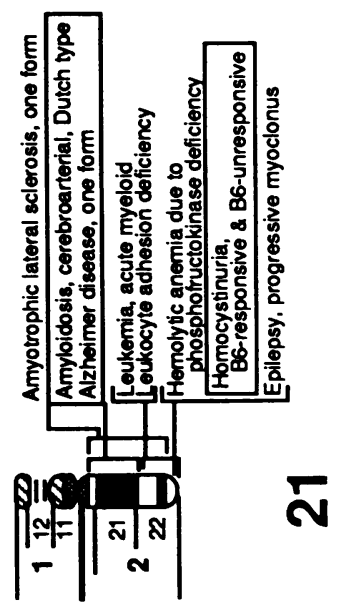


Table 1 The morbid anatomy of the human genome (by chromosome).

\begin{tabular}{|c|c|c|c|c|c|c|c|}
\hline Location & Symbol & Status & Title & MIM\# & Method & Disorder & Mouse \\
\hline 1pter-p36.13 & ENO1, PPH & C & Enolase-1, alpha & 172430 & $\mathrm{~S}, \mathrm{~F}, \mathrm{R}, \mathrm{REa}$ & Enolase deficiency (1) & $4($ Eno-1) \\
\hline 1p36.3-p34.1 & C1QA & C & $\begin{array}{l}\text { Complement component-1, q } \\
\text { subcomponent, alpha } \\
\text { polypeptide }\end{array}$ & 120550 & $\mathrm{REa}, \mathrm{REb}$ & ?C1q deficiency (1) & \\
\hline $1 \mathrm{p} 36.3-\mathrm{p} 34.1$ & $\mathrm{C} 1 \mathrm{QB}$ & $\mathrm{C}$ & $\begin{array}{l}\text { Complement component }-1, \mathrm{q} \\
\text { subcomponent, beta polypeptide }\end{array}$ & 120570 & $\mathrm{REa}, \mathrm{REb}$ & ?C1q deficiency (1) & $(\mathrm{Clqb})$ \\
\hline $1 \mathrm{p} 36.3-\mathrm{p} 36.2$ & PLOD & $\mathbf{P}$ & $\begin{array}{l}\text { Procollagen-lysine, 2-oxoglutarate } \\
\text { 5-dioxygenase (lysine } \\
\text { hydroxylase) }\end{array}$ & 153454 & $\mathrm{REa}, \mathrm{A}$ & $\begin{array}{l}\text { Ehlers-Danlos syndrome, type VI, } \\
225400 \text { (1) }\end{array}$ & \\
\hline $1 \mathrm{p} 36.2-\mathrm{p} 36.1$ & NB, NBS & C & $\begin{array}{l}\text { Neuroblastoma (neuroblastoma } \\
\text { suppressor) }\end{array}$ & 256700 & $\mathrm{Ch}, \mathrm{D}$ & Neuroblastoma (2) & \\
\hline $1 \mathrm{p} 36.2-\mathrm{p} 34$ & EL1 & C & Elliptocytosis-1 (protein 4.1) & 130500 & $\mathrm{~F}, \mathrm{REb}$ & Elliptocytosis-1 (3) & $4($ Elp-1) \\
\hline $1 \mathrm{p} 36.2-\mathrm{p} 34$ & EKV & $\mathrm{C}$ & Erythrokeratodermia variabilis & 133200 & F & Erythrokeratodermia variabilis (2) & \\
\hline $1 \mathrm{p} 36.2-\mathrm{p} 34$ & RH & $\mathrm{C}$ & $\begin{array}{l}\text { RHESUS BLOOD GROUP } \\
\text { CLUSTER }\end{array}$ & 111700 & F, D, Fd, A & $\begin{array}{l}\text { Erythroblastosis fetalis (1); } \\
\text { ?Rh-null hemolytic anemia (1) }\end{array}$ & \\
\hline 1p36.1-p34 & ALPL, HOPS & $\mathrm{C}$ & $\begin{array}{l}\text { Alkaline phosphatase, } \\
\text { liver/bone/kidney }\end{array}$ & 171760 & $\begin{array}{l}\text { S, H, Fd, F, } \\
\text { A }\end{array}$ & $\begin{array}{l}\text { Hypophosphatasia, infantile } 241500 \\
\text { (3); ?Hypophosphatasia, adult } \\
146300 \text { (1) }\end{array}$ & $4(\mathrm{Akp}-2)$ \\
\hline $1 \mathrm{p} 36$ & $\mathrm{BRCD} 2$ & $P$ & Breast cancer, ductal & 211420 & $\mathrm{Ch}, \mathrm{F}, \mathrm{D}$ & Breast cancer, ductal (2) & \\
\hline $1 \mathrm{p} 36$ & $\begin{array}{l}\text { CMM, } \\
\text { HCMM, } \\
\text { MLM, } \\
\text { DNS }\end{array}$ & I & $\begin{array}{l}\text { Malignant melanoma, cutaneous } \\
\text { (dysplastic nevus syndrome) }\end{array}$ & 155600 & $\mathrm{~F}, \mathrm{Fd}$ & $\begin{array}{l}\text { ?Malignant melanoma, cutaneous } \\
\text { (2) }\end{array}$ & \\
\hline $1 \mathrm{p} 36-\mathrm{p} 35$ & GALE & C & UDP galactose-4-epimerase & 230350 & S, LD & Galactose epimerase deficiency (1) & \\
\hline $1 \mathrm{p} 34$ & $\begin{array}{l}\text { FUCA1, } \\
\text { FUCA }\end{array}$ & $\mathrm{C}$ & Fucosidase, alpha-L- 1 , tissue & 230000 & $\begin{array}{l}\mathrm{S}, \mathrm{F}, \mathrm{R}, \mathrm{A} \\
\mathrm{REa}\end{array}$ & Fucosidosis (1) & 4(Fuca) \\
\hline $1 \mathrm{p} 34$ & UROD & $\mathrm{C}$ & Uroporphyrinogen decarboxylase & 176100 & $\mathrm{~S}, \mathrm{~A}, \mathrm{REa}$ & $\begin{array}{l}\text { Porphyria cutanea tarda (1); } \\
\text { Porphyria, hepatoerythropoietic } \\
\text { (1) }\end{array}$ & 4 (Urod) \\
\hline $1 \mathrm{p} 32$ & C8A & C & $\begin{array}{l}\text { Complement component } 8 \text {, alpha } \\
\text { polypeptide }\end{array}$ & 120950 & $\mathrm{~F}, \mathrm{~A}, \mathrm{Ch}$ & C8 deficiency, type I (2) & \\
\hline $1 \mathrm{p} 32$ & $\mathrm{C} 8 \mathrm{~B}$ & $\mathrm{C}$ & $\begin{array}{l}\text { Complement component } 8 \text {, beta } \\
\text { polypeptide }\end{array}$ & 120960 & $\mathrm{~F}, \mathrm{~A}, \mathrm{Ch}, \mathrm{H}$ & C8 deficiency, type II (2) & $4(\mathrm{C} 8 \mathrm{~b})$ \\
\hline $1 \mathrm{p} 32$ & CLN1 & $\mathbf{P}$ & $\begin{array}{l}\text { Ceroid lipofuscinosis, neuronal-1, } \\
\text { infantile }\end{array}$ & 256730 & Fd, LD & $\begin{array}{l}\text { Ceroid lipofuscinosis, neuronal-1, } \\
\text { infantile (2) }\end{array}$ & \\
\hline $1 \mathrm{p} 32$ & $\begin{array}{l}\text { TAL1, } \\
\text { TCL5, } \\
\text { SCL }\end{array}$ & $\mathrm{C}$ & $\begin{array}{l}\text { T-cell leukemia/lymphoma-5 } \\
\text { (stem-cell leukemia) }\end{array}$ & 187040 & $\mathrm{Ch}, \mathrm{RE}$ & $\begin{array}{l}\text { Leukemia-1, T-cell acute } \\
\text { lymphoblastic ( } 3)\end{array}$ & $4(\mathrm{Scl})$ \\
\hline $1 \mathrm{p} 31$ & $\begin{array}{l}\text { ACADM, } \\
\text { MCAD }\end{array}$ & $\mathbf{P}$ & $\begin{array}{l}\text { Acyl-CoA dehydrogenase, medium } \\
\text { chain }\end{array}$ & 201450 & $\mathrm{REa}, \mathrm{A}$ & $\begin{array}{l}\text { Acyl-CoA dehydrogenase, medium } \\
\text { chain, deficiency of (1) }\end{array}$ & 8(Acadm) \\
\hline $1 \mathrm{p} 31$ & $\begin{array}{l}\text { DBT, } \\
\text { BCATE2 }\end{array}$ & C & $\begin{array}{l}\text { Dihydrolipoamide branched chain } \\
\text { transacylase (E2 component of } \\
\text { branched chain keto acid } \\
\text { dehydrogenase complex) }\end{array}$ & 248610 & $\mathrm{REa}, \mathrm{A}$ & $\begin{array}{l}\text { Maple syrup urine disease, type } 2 \\
\text { (3) }\end{array}$ & \\
\hline $1 \mathrm{p} 22.1-\mathrm{q} 21.1$ & CD3Z, TCRZ & $\mathbf{P}$ & $\begin{array}{l}\text { Antigen CD3Z, zeta polypeptide } \\
\text { (TiT3 complex) }\end{array}$ & 186780 & $\mathrm{REa}, \mathrm{A}$ & CD3, zeta chain, deficiency (1) & $1(\mathrm{~T} 3 \mathrm{z})$ \\
\hline $1 \mathrm{p} 21$ & AGL & $\mathbf{P}$ & $\begin{array}{l}\text { Amylo-1,6-glucosidase, } \\
\text { 4-alpha-glucanotransferase } \\
\text { (glycogen debranching enzyme) }\end{array}$ & 232400 & REc, A & Glycogen storage disease- 3 (3) & \\
\hline $1 \mathrm{p} 21-\mathrm{p} 13$ & AMPD1 & $\mathbf{P}$ & $\begin{array}{l}\text { Adenosine monophosphate } \\
\text { deaminase-1 }\end{array}$ & 102770 & $\mathrm{REa}, \mathrm{A}$ & $\begin{array}{l}\text { Myoadenylate deaminase deficiency } \\
\text { (1) }\end{array}$ & 3(Ampd-1) \\
\hline $1 \mathrm{p} 21-\mathrm{p} 13$ & AMPD2 & $\mathrm{L}$ & $\begin{array}{l}\text { Adenosine monophosphate } \\
\text { deaminase-2 }\end{array}$ & 102771 & $\mathrm{H}$ & $\begin{array}{l}\text { [AMP deaminase deficiency, } \\
\text { erythrocyte] (1) }\end{array}$ & 3(Ampd-2) \\
\hline $1 \mathrm{p} 21-\mathrm{p} 13$ & CSF1, MCSF & C & $\begin{array}{l}\text { Colony-stimulating factor-1 } \\
\text { (macrophage) }\end{array}$ & 120420 & $\mathrm{~A}, \mathrm{REa}, \mathrm{H}$ & ?Osteopetrosis, 259700 (1) & $3(\mathrm{Csfm})$ \\
\hline $1 \mathrm{p} 13.1$ & $\begin{array}{l}\text { HSD3B2, } \\
\text { HSD3B, } \\
\text { HSDB3 }\end{array}$ & C & $\begin{array}{l}\text { Hydroxy-delta- } 5 \text {-steroid } \\
\text { dehydrogenase, } 3 \text { beta- and } \\
\text { steroid delta-isomerase, type } 2\end{array}$ & 201810 & A & Adrenal hyperplasia II (3) & $3(\mathrm{Hsd} 3 \mathrm{~b})$ \\
\hline $1 \mathrm{p} 13$ & TSHB & C & $\begin{array}{l}\text { Thyroid-stimulating hormone, beta } \\
\text { polypeptide }\end{array}$ & 188540 & $\mathrm{REa}, \mathrm{RE}, \mathrm{Fd}$ & Hypothyroidism, nongoitrous (1) & 3 (Tshb) \\
\hline 1p13-p11 & CPT1 & C & Carnitine palmitoyltransferase I & 255120 & $\mathrm{REa}, \mathrm{A}$ & $\begin{array}{l}\text { Myopathy due to CTPase } \\
\text { deficiency (1); Hyperammonemia } \\
\text { due to CTPase deficiency (1) }\end{array}$ & \\
\hline 1p11-qter & $\begin{array}{l}\text { EPHX, } \\
\text { EPOX }\end{array}$ & $\mathbf{P}$ & $\begin{array}{l}\text { Epoxide hydroxylase, microsomal } \\
\text { (epoxide hydrolase) }\end{array}$ & 132810 & REa & $\begin{array}{l}\text { ?Fetal hydantoin syndrome (1); } \\
\text { Diphenylhydantoin toxicity (1) }\end{array}$ & $1(\mathrm{Eph}-1)$ \\
\hline 1 cen-q32 & PFKM & $\mathbf{P}$ & Phosphofructokinase, muscle type & 232800 & S & Glycogen storage disease VII (1) & \\
\hline 1q & FMO2 & $\mathbf{P}$ & $\begin{array}{l}\text { Flavin-containing monooxygenase } \\
2 \text { (adult liver) }\end{array}$ & 136131 & Psh & [Fish-odor syndrome] (1) & \\
\hline 1q2 & CAE & C & Cataract, zonular pulverulent & 116200 & F & Cataract, zonular pulverulent (2) & \\
\hline 1q21 & FLG & $\mathbf{P}$ & Filaggrin & 135940 & REa, A & ?Ichthyosis vulgaris, 146700 (1) & \\
\hline 1q21 & GBA & C & Glucosidase, beta; acid & 230800 & S, A, D & Gaucher disease (1) & 3(Gba) \\
\hline $1 \mathrm{q} 21$ & $\begin{array}{l}\text { PKLR, PK1, } \\
\text { PKR }\end{array}$ & $\mathrm{C}$ & $\begin{array}{l}\text { Pyruvate kinase, liver and RBC } \\
\text { type }\end{array}$ & 266200 & $\mathrm{REa}, \mathrm{A}$ & PK deficiency hemolytic anemia (1) & \\
\hline $1 \mathrm{q} 21$ & SPTA1 & C & Spectrin, alpha, erythrocytic-1 & 182860 & $\mathrm{REa}, \mathrm{A}, \mathrm{Fd}$ & $\begin{array}{l}\text { Elliptocytosis-2 (2); } \\
\text { Pyropoikilocytosis (1); } \\
\text { Spherocytosis, recessive (1) }\end{array}$ & 1 (Spna-1) \\
\hline $1 q 21-q 22$ & FY & C & Duffy blood group & 110700 & $\mathrm{~F}, \mathrm{Fc}, \mathrm{Fd}$ & $\begin{array}{l}\text { \{Vivax malaria, susceptibility to }\} \\
\text { (1) }\end{array}$ & \\
\hline $1 q 21-q 23$ & APCS, SAP & $\mathrm{C}$ & Amyloid P component, serum & 104770 & $\mathrm{REa}, \mathrm{A}, \mathrm{Fd}$ & $\begin{array}{l}\text { \{?Amyloidosis, secondary, } \\
\text { susceptibility to }(1)\end{array}$ & 1 (Sap) \\
\hline $1 q 21-q 23$ & NEM1 & $\mathbf{P}$ & Nemaline myopathy & 161800 & $\mathrm{Fd}$ & Nemaline myopathy (2) & \\
\hline 1q21-q24 & GRMP & C & $\begin{array}{l}\text { Granulocyte membrane protein } \\
(140 \mathrm{kD} \text {, antigen CD62) }\end{array}$ & 173610 & REn, A & $\begin{array}{l}\text { Platelet alpha/delta storage pool } \\
\text { deficiency (1) }\end{array}$ & 1 (Grmp) \\
\hline $1 \mathrm{q} 21.2-\mathrm{q} 23$ & СMT1B & $\mathrm{C}$ & $\begin{array}{l}\text { Charcot-Marie-Tooth disease, slow } \\
\text { nerve conduction type Ib }\end{array}$ & 118200 & $\mathrm{~F}, \mathrm{Fd}, \mathrm{D}$ & $\begin{array}{l}\text { Charcot-Marie-Tooth neuropathy, } \\
\text { slow nerve conduction type Ib } \\
\text { (2) }\end{array}$ & \\
\hline $1 \mathrm{q} 23$ & F5 & $\mathrm{C}$ & Coagulation factor $\mathrm{V}$ & 227400 & $\begin{array}{l}\text { REa, A, Fd, } \\
\text { REn }\end{array}$ & Factor V deficiency $(1)$ & $1(\mathrm{Cf}-5)$ \\
\hline
\end{tabular}


Table 1-contd

\begin{tabular}{|c|c|c|c|c|c|c|c|}
\hline Location & Symbol & Status & Title & MIM\# & Method & Disorder & Mouse \\
\hline $1 \mathrm{q} 23$ & $\begin{array}{l}\text { FCGR3, } \\
\text { CD16, } \\
\text { IGFR3 }\end{array}$ & C & $\begin{array}{l}\text { Fc fragment of IgG, low affinity } \\
\text { III, receptor for (CD16) } \\
\text { (Immunoglobulin G Fc receptor } \\
\text { III) }\end{array}$ & 146740 & REb, REn & $\begin{array}{l}\text { Lupus erythematosus, systemic, } \\
152700 \text { (1); Neutropenia, } \\
\text { immune (2) }\end{array}$ & \\
\hline $1 \mathrm{q} 23$ & PBX1 & $\mathrm{C}$ & $\begin{array}{l}\text { Pre-B cell leukemia transcription } \\
\text { factor-1 }\end{array}$ & 176310 & $\mathrm{Ch}, \mathrm{A}$ & Leukemia, acute pre-B-cell (2) & $1(\mathrm{Pbx})$ \\
\hline $1 q 23-q 25$ & AT3 & $\mathrm{C}$ & Antithrombin III & 107300 & $\begin{array}{l}\text { F, D, A, REa, } \\
\text { Fd }\end{array}$ & Antithrombin III deficiency (3) & $1($ At-3) \\
\hline $1 \mathrm{q} 25$ & NCF2 & $\mathrm{C}$ & Neutrophil cytosolic factor-2 & 233710 & REa, A & $\begin{array}{l}\text { Chronic granulomatous disease due } \\
\text { to deficiency of NCF-2 (1) }\end{array}$ & $1(\mathrm{Ncf}-2)$ \\
\hline $1 q 31-q 32.1$ & F13B & $\mathrm{C}$ & $\begin{array}{l}\text { Coagulation factor XIII, B } \\
\text { polypeptide }\end{array}$ & 134580 & Fd, A, RE & Factor XIIIB deficiency (1) & \\
\hline 1q32 & CR1, C3BR & $\mathrm{C}$ & $\begin{array}{l}\text { Complement component }(3 \mathrm{~b} / 4 \mathrm{~b}) \\
\text { receptor-1 }\end{array}$ & 120620 & F, REa, A, & CR1 deficiency (1); ?SLE (1) & \\
\hline $1 \mathrm{q} 32$ & $\mathrm{HF}, \mathrm{CFH}$ & C & Complement component $\mathrm{H}$ & 134370 & $\begin{array}{l}\mathrm{F}, \mathrm{REa}, \mathrm{RE} \text {, } \\
\mathrm{H}\end{array}$ & Factor $\mathbf{H}$ deficiency (1) & $1(\mathrm{Cfh})$ \\
\hline $1 \mathrm{q} 32$ & USH2 & C & $\begin{array}{l}\text { Usher syndrome-2 (autosomal } \\
\text { recessive, mild) }\end{array}$ & 276901 & Fd & Usher syndrome, type 2 (2) & \\
\hline $1 \mathrm{q} 32$ & $\begin{array}{l}\text { VWS, LPS, } \\
\text { PIT, } \\
\text { VDWS }\end{array}$ & C & $\begin{array}{l}\text { Lip pit syndrome (van der Woude } \\
\text { syndrome) }\end{array}$ & 119300 & $\mathrm{Ch}, \mathrm{Fd}$ & van der Woude syndrome (2) & \\
\hline $1 q 41-q 42$ & $\begin{array}{l}\text { ADPRT, } \\
\text { PPOL, } \\
\text { PARP }\end{array}$ & C & $\begin{array}{l}\text { Poly-ADP-ribose polymerase } \\
\text { (NAD }(+) \\
\text { ADP-ribosyltransferase) }\end{array}$ & 173870 & $\mathrm{REa}, \mathrm{A}$ & $\begin{array}{l}\text { ?Fanconi anemia (1); ?Xeroderma } \\
\text { pigmentosum (1) }\end{array}$ & \\
\hline $1 \mathrm{q} 42.1$ & $\mathrm{FH}$ & C & Fumarate hydratase & 136850 & S, R, D & Fumarase deficiency (1) & \\
\hline Chr.1 & HSD11 & $\mathbf{P}$ & $\begin{array}{l}\text { 11-beta-hydroxysteroid } \\
\text { dehydrogenase }\end{array}$ & 218030 & REa & $\begin{array}{l}\text { 11-beta-hydroxysteroid } \\
\text { dehydrogenase deficiency (1) }\end{array}$ & \\
\hline 2pter-p12 & TPO, TPX & C & Thyroid peroxidase & 274500 & REa, A & $\begin{array}{l}\text { Thyroid iodine peroxidase } \\
\text { deficiency (1) }\end{array}$ & $12(\mathrm{Tpo})$ \\
\hline 2p25 & POMC & $\mathrm{C}$ & Proopiomelanocortin & 176830 & REa & ACTH deficiency (1) & 12(Pomc-1) \\
\hline $2 \mathrm{p} 24$ & APOB & C & Apolipoprotein B & 107730 & REa, A & $\begin{array}{l}\text { Hypobetalipoproteinemia (1); } \\
\text { ?Abetalipoproteinemia (1); } \\
\text { Hyperbetalipoproteinemia (1); } \\
\text { Apolipoprotein B-100, defective } \\
\text { (1) }\end{array}$ & 12(Apob) \\
\hline 2p21 & HPC & $\mathbf{L}$ & Holoprosencephaly & 157170 & $\mathrm{Ch}$ & ?Holoprosencephaly (2) & \\
\hline 2p12 & IGKC & C & $\begin{array}{l}\text { Constant region of kappa light } \\
\text { chain }\end{array}$ & 147200 & REa, A & [Kappa light chain deficiency] (1) & \\
\hline $2 p$ & CPS1 & $\mathbf{P}$ & $\begin{array}{l}\text { Carbamoylphosphate synthetase I } \\
\text { (mitochondrial CPS) }\end{array}$ & 237300 & REa & $\begin{array}{l}\text { Carbamoylphosphate synthetase I } \\
\text { deficiency (1) }\end{array}$ & \\
\hline 2q13-q14 & PROC & C & Protein C & 176860 & REa, A & Protein C deficiency (1) & \\
\hline $2 q 14-q 21$ & LCO, LCA & $\mathbf{P}$ & $\begin{array}{l}\text { Liver cancer oncogene (oncogene } \\
\text { LCA) }\end{array}$ & 165320 & REa, REb, A & ?Hepatocarcinoma (1) & \\
\hline $2 \mathrm{q} 21$ & ERCC3, XPB & C & $\begin{array}{l}\text { Excision-repair, complementing } \\
\text { defective, in Chinese hamster, } \\
\text { number } 3\end{array}$ & 133510 & S, A & $\begin{array}{l}\text { Xeroderma pigmentosum, group B } \\
\text { (1) }\end{array}$ & \\
\hline $2 \mathrm{q} 31$ & COL3A1 & C & Collagen III, alpha-1 polypeptide & 120180 & REa, A & $\begin{array}{l}\text { Ehlers-Danlos syndrome, type IV } \\
\text { (3); Aneurysm, familial (1) }\end{array}$ & $1($ Col3a-1) \\
\hline $2 \mathrm{q} 31$ & GAD1 & C & Glutamate decarboxylase-1, brain & 266100 & $\mathrm{REa}, \mathrm{H}, \mathrm{A}$ & $\begin{array}{l}\text { ?Pyridoxine dependency with } \\
\text { seizures (1) }\end{array}$ & $2(\mathrm{Gad}-1)$ \\
\hline $2 \mathrm{q} 31.2$ & OCA & L & $\begin{array}{l}\text { Oculocutaneous albinism, type II } \\
\text { (tyrosinase-positive) }\end{array}$ & 203200 & $\mathrm{Ch}$ & $\begin{array}{l}\text { ?Oculocutaneous albinism, type II } \\
\text { (2) }\end{array}$ & \\
\hline $2 q 33-q 35$ & CRYG1 & C & Crystallin, gamma polypeptide 1 & 123660 & REa, A & Cataract, Coppock-like (3) & 1 (Cryg-1) \\
\hline 2q33-qter & CYP27, CTX & $\mathbf{P}$ & Sterol 27-hydroxylase & 213700 & REa & $\begin{array}{l}\text { Cerebrotendinous xanthomatosis } \\
\text { (2) }\end{array}$ & 1(Cyp27) \\
\hline $2 \mathrm{q} 34$ & TCL4 & $\mathbf{P}$ & T-cell leukemia/lymphoma-4 & 186860 & $\mathrm{Ch}, \mathrm{RE}$ & Leukemia/lymphoma, T-cell (2) & \\
\hline 2q34-q35 & $\begin{array}{l}\text { ACADL, } \\
\text { LCAD }\end{array}$ & $\mathbf{P}$ & $\begin{array}{l}\text { Acyl-CoA dehydrogenase, } \\
\text { long-chain }\end{array}$ & 201460 & A & $\begin{array}{l}\text { Acyl-CoA dehydrogenase, long } \\
\text { chain, deficiency of (1) }\end{array}$ & \\
\hline 2q34-q36 & FN1 & C & Fibronectin-1 & 135600 & S, REa, A & $\begin{array}{l}\text { ?Ehlers-Danlos syndrome, type } \mathrm{X} \\
\text { (1) }\end{array}$ & $1($ Fn-1) \\
\hline 2q35 & DES & $\mathbf{P}$ & Desmin & 125660 & $\mathrm{REa}, \mathrm{A}$ & ?Cardiomyopathy (1) & 1 (Des) \\
\hline $2 q 35$ & $\begin{array}{l}\text { PAX3, WS1, } \\
\text { HUP2 }\end{array}$ & $\mathrm{C}$ & $\begin{array}{l}\text { Paired box homeotic gene- } 3 \\
\text { (Waardenburg syndrome, type I) }\end{array}$ & 193500 & $\mathrm{Ch}, \mathrm{Fd}, \mathrm{H}, \mathrm{A}$ & Waardenburg syndrome, type I (3) & $1(\mathrm{Sp})$ \\
\hline $2 q 36-q 37$ & $\begin{array}{l}\text { AGXT, } \\
\text { AGT, } \\
\text { SPAT }\end{array}$ & $\mathbf{P}$ & $\begin{array}{l}\text { Alanine:glyoxylate } \\
\text { aminotransferase, liver-specific } \\
\text { peroxisomal }\end{array}$ & 259900 & A, REa & Oxalosis I (1) & \\
\hline $2 \mathrm{q} 36-\mathrm{q} 37$ & GCG & $\mathrm{C}$ & Glucagon & 138030 & $\mathrm{REa}, \mathrm{A}$ & [?Hyperproglucagonemia] (1) & $2(\mathrm{Gcg})$ \\
\hline 2q37 & RMSA & C & Rhabdomyosarcoma, alveolar & 268220 & Ch & Rhabdomyosarcoma, alveolar (2) & \\
\hline Chr.2 & $\begin{array}{l}\text { LCT, LAC, } \\
\text { LPH }\end{array}$ & C & $\begin{array}{l}\text { Lactase (lactase-phlorizin } \\
\text { hydrolase) }\end{array}$ & 223000 & REa, Fd & $\begin{array}{l}\text { ?Lactase deficiency, congenital (1); } \\
\text { ?Lactase deficiency, adult, } \\
223100 \text { (1) }\end{array}$ & \\
\hline Chr.2 & $\begin{array}{l}\text { UGT1, } \\
\text { GNT1 }\end{array}$ & $\mathbf{P}$ & $\begin{array}{l}\text { UDP-glucuronosyltransferase-1 } \\
\text { family }\end{array}$ & 191740 & REa & $\begin{array}{l}\text { Crigler-Najjar syndrome, type I, } \\
218800 \text { (1); ? (Gilbert syndrome, } \\
\text { 143500)(1) }\end{array}$ & 1(Ugt-1) \\
\hline 3 p26-p25 & VHL & C & von Hippel-Lindau syndrome & 193300 & Fd, D & von Hippel-Lindau syndrome (2) & \\
\hline $3 \mathrm{p} 24.3$ & $\begin{array}{l}\text { THRB, } \\
\text { THR1, } \\
\text { ERBA2 }\end{array}$ & C & $\begin{array}{l}\text { Thyroid hormone receptor, beta } \\
\text { (oncogene ERBA2) }\end{array}$ & 190160 & $\begin{array}{l}\text { REa, A, RE, } \\
\text { Fd }\end{array}$ & $\begin{array}{l}\text { Thyroid hormone resistance, } \\
274300,188570 \text { (3) }\end{array}$ & \\
\hline $3 p 23-p 22$ & ACAA & $\mathbf{P}$ & $\begin{array}{l}\text { Peroxisomal 3-oxoacyl-CoA } \\
\text { thiolase }\end{array}$ & 261510 & $\mathrm{REa}, \mathrm{A}$ & Pseudo-Zellweger syndrome (1) & \\
\hline $3 \mathrm{p} 23-\mathrm{p} 21$ & $\begin{array}{l}\text { SCLC1, } \\
\text { SCCL }\end{array}$ & C & Small-cell cancer of lung & 182280 & $\mathrm{Ch}, \mathrm{D}$ & Small-cell cancer of lung (2) & \\
\hline $3 \mathrm{p} 21$ & COL7A1 & $\mathbf{P}$ & Collagen VII, alpha-1 polypeptide & 120120 & REa, A & $\begin{array}{l}\text { Epidermolysis bullosa dystrophica, } \\
\text { dominant, } 131750(3) \text {; } \\
\text { Epidermolysis bullosa } \\
\text { dystrophica, recessive, } 226600 \text { (3) }\end{array}$ & \\
\hline $3 p 21-p 14.2$ & GLB1 & C & Galactosidase, beta-1 & 230500 & S, EM & $\begin{array}{l}\text { GM1-gangliosidosis (1); } \\
\text { Mucopolysaccharidosis IVB (1) }\end{array}$ & $9(\mathrm{Bgl})$ \\
\hline
\end{tabular}


Table 1-contd

\begin{tabular}{|c|c|c|c|c|c|c|c|}
\hline Location & Symbol & Status & Title & MIM\#\# & Method & Disorder & Mouse \\
\hline $3 \mathrm{p} 14.2$ & $\mathrm{RCCl}, \mathrm{RCC}$ & C & Renal cell carcinoma & 144700 & $\mathrm{Fc}, \mathrm{Ch}$ & Renal cell carcinoma (2) & \\
\hline 3p14-qter & HRG & $\mathbf{P}$ & Histidine-rich glycoprotein & 142640 & REa & $\begin{array}{l}\text { Thrombophilia due to elevated } \\
\text { HRG (2) }\end{array}$ & \\
\hline $3 \mathrm{p} 11.1-\mathrm{q} 11.2$ & $\begin{array}{l}\text { PROS1, PSA, } \\
\text { PROS }\end{array}$ & $\mathrm{C}$ & Protein S, alpha & 176880 & REa & Protein $\mathrm{S}$ deficiency (1) & \\
\hline 3 cen-q22 & MER6, RHN & $\mathbf{P}$ & $\mathrm{Rh}$-null, regulator type & 268150 & $S$ & $\mathrm{Rh}$-null disease $(1)$ & \\
\hline $3 q$ & PIT1 & $\mathbf{P}$ & $\begin{array}{l}\text { Pituitary-specific transcription } \\
\text { factor Pit-1 }\end{array}$ & 173110 & $\mathrm{Fd}$ & ?Panhypopituitarism (1) & 16(Pit-1,dw) \\
\hline $3 q 11-q 12$ & GPX1 & $\mathrm{C}$ & Glutathione peroxidase-1 & 138320 & S, REa & $\begin{array}{l}\text { Hemolytic anemia due to } \\
\text { glutathione peroxidase deficiency } \\
\text { (1) }\end{array}$ & \\
\hline $3 q 13$ & $\begin{array}{l}\text { UMPS, } \\
\text { OPRT }\end{array}$ & $\mathrm{C}$ & $\begin{array}{l}\text { Orotate } \\
\text { phosphoribosyltransferase/OMP } \\
\text { decarboxylase (UMP synthase) }\end{array}$ & 258900 & S, A & Oroticaciduria (1) & \\
\hline $3 q^{2}$ & BPES & $\mathbf{P}$ & $\begin{array}{l}\text { Blepharophimosis, epicanthus } \\
\text { inversus and ptosis }\end{array}$ & 110100 & $\mathrm{Ch}$ & $\begin{array}{l}\text { Blepharophimosis, epicanthus } \\
\text { inversus and ptosis (2) }\end{array}$ & \\
\hline $3 \mathrm{q} 21$ & TF & $\mathrm{C}$ & Transferrin & 190000 & $\begin{array}{l}\text { S, H, REa, D, } \\
\text { A }\end{array}$ & Atransferrinemia (1) & 9 (Trf) \\
\hline $3 q 21-q 22$ & PCCB & $\mathrm{C}$ & $\begin{array}{l}\text { Propionyl CoA carboxylase, beta } \\
\text { polypeptide }\end{array}$ & 232050 & $\mathrm{REa}, \mathrm{A}, \mathrm{D}$ & $\begin{array}{l}\text { Propionicacidemia, type II or pccB } \\
\text { type (1) }\end{array}$ & \\
\hline $3 q 21-q 23$ & LTF & $\mathrm{C}$ & Lactotransferrin & 150210 & REa, A & $\begin{array}{l}\text { ?Lactoferrin-deficient neutrophils, } \\
245480 \text { (1) }\end{array}$ & $9(\mathrm{Ltf})$ \\
\hline $3 q 21-q 24$ & $\mathrm{CP}$ & $\mathrm{C}$ & Ceruloplasmin & 117700 & $\mathrm{~F}, \mathrm{H}, \mathrm{REa}, \mathrm{A}$ & $\begin{array}{l}\text { [Hypoceruloplasminemia, } \\
\text { hereditary] (1) }\end{array}$ & $9(\mathrm{Cp})$ \\
\hline $3 q 21-q 24$ & FHH, HHC & $\mathbf{P}$ & $\begin{array}{l}\text { Familial hypocalciuric } \\
\text { hypercalcemia }\end{array}$ & 145980 & $\mathrm{Fd}$ & $\begin{array}{l}\text { Hypercalcemia, hypocalciuric, } \\
\text { familial (2) }\end{array}$ & \\
\hline $3 q 21-q 24$ & RHO, RP4 & $\mathrm{C}$ & Rhodopsin & 180380 & $\mathrm{REa}, \mathrm{A}, \mathrm{Fd}$ & $\begin{array}{l}\text { Retinitis pigmentosa, autosomal } \\
\text { dominant (3) }\end{array}$ & 6(Rho) \\
\hline $3 q 25-q 26$ & SI & $\mathbf{P}$ & Sucrase-isomaltase & 222900 & $\mathrm{REa}, \mathrm{A}, \mathrm{Fd}$ & Sucrose intolerance (1) & \\
\hline 3q26-qter & KNG & C & Kininogen & 228960 & Psh, A & [Kininogen deficiency] (1) & \\
\hline $3 q 26.1-q 26.2$ & BCHE, CHE 1 & C & Pseudocholinesterase-1 & 177400 & $\mathrm{~F}, \mathrm{D}, \mathrm{A}$ & Postanesthetic apnea (1) & \\
\hline $3 q 26.3$ & CDL & L & Cornelia de Lange syndrome & 122470 & $\mathrm{Ch}$ & ?Cornelia de Lange syndrome (2) & \\
\hline Chr.3 & TRH & $\mathbf{P}$ & Thyrotropin-releasing hormone & 275120 & REa & $\begin{array}{l}\text { Thyrotropin-releasing hormone } \\
\text { deficiency (1) }\end{array}$ & \\
\hline $4 \mathrm{p} 16.3$ & HD & C & Huntington disease & 143100 & Fd & Huntington disease (2) & \\
\hline $4 \mathrm{p} 16.3$ & IDUA, IDA & $\mathbf{P}$ & Alpha-L-iduronidase & 252800 & $\mathrm{REa}, \mathrm{A}, \mathrm{S}$ & $\begin{array}{l}\text { Hurler syndrome (1); } \\
\text { Mucopolysaccharidosis I (1); } \\
\text { Hurler-Scheie syndrome (1); } \\
\text { Scheie syndrome (1) }\end{array}$ & \\
\hline $4 \mathrm{p} 16.1$ & HOX7 & $\mathbf{P}$ & Homeo box-7 & 142983 & $\mathrm{REa}, \mathrm{A}, \mathrm{D}, \mathrm{Fd}$ & ?Wolf-Hirschhorn syndrome (3) & $5($ Hox -7$)$ \\
\hline $4 \mathrm{p} 15.31$ & $\begin{array}{l}\text { QDPR, } \\
\text { DHPR }\end{array}$ & C & Quinoid dihydropteridine reductase & 261630 & S, A, REa, D & $\begin{array}{l}\text { Phenylketonuria due to } \\
\text { dihydropteridine reductase } \\
\text { deficiency (1) }\end{array}$ & 5(Qdpr) \\
\hline $4 q 11-q 12$ & KIT, PBT & C & $\begin{array}{l}\text { Hardy-Zuckerman } 4 \text { feline sarcoma } \\
\text { (v-kit) oncogene }\end{array}$ & 164920 & $\begin{array}{l}\text { REa, A, H, } \\
\quad \text { Ch, H, REn }\end{array}$ & Piebaldism (3) & $5(\mathrm{Kit} ; \mathrm{W})$ \\
\hline $4 q 11-q 13$ & AFP, HPAFP & C & Alpha-fetoprotein & 104150 & $\mathrm{H}, \mathrm{A}, \mathrm{Fd}, \mathrm{F}$ & $\begin{array}{l}\text { [AFP deficiency, congenital] (1); } \\
\text { [Hereditary persistence of } \\
\text { alpha-fetoprotein] (3) }\end{array}$ & 5(Afp) \\
\hline $4 q 11-q 13$ & ALB & C & Albumin & 103600 & F, A, REa & $\begin{array}{l}\text { Analbuminemia (1); } \\
\text { [Dysalbuminemic } \\
\text { hyperthyroxinemia] (1); } \\
\text { [Dysalbuminemic hyperzincemia] } \\
\text { (1) }\end{array}$ & 5(Alb-1) \\
\hline $4 q 11-q 13$ & JPD, JP & $\mathbf{P}$ & Periodontitis, juvenile & 170650 & $\mathbf{F}$ & Periodontitis, juvenile (2) & \\
\hline $4 q 13-q 21$ & DGI1 & C & Dentinogenesis imperfecta-1 & 125490 & $\mathrm{~F}, \mathrm{Fd}$ & Dentinogenesis imperfecta-1 (2) & \\
\hline $4 q 21$ & $\begin{array}{l}\text { IGJ, IGCJ, } \\
\text { JCH }\end{array}$ & $\mathbf{P}$ & $\begin{array}{l}\mathrm{J} \text { region of immunoglobulin heavy } \\
\text { chain }\end{array}$ & 147790 & $\mathrm{REa}, \mathrm{A}$ & $\begin{array}{l}\text { ?Leukemia, acute lymphocytic, } \\
\text { with } 4 / 11 \text { translocation ( } 3 \text { ) }\end{array}$ & $5(\mathrm{Ig} j)$ \\
\hline $4 q 21-q 23$ & GNPTA & $\mathbf{P}$ & $\begin{array}{l}\text { N-acetyl-alpha-glucosaminyl- } \\
\text { phosphotransferase }\end{array}$ & 252500 & F, S, D & $\begin{array}{l}\text { Mucolipidosis II (1); Mucolipidosis } \\
\text { III (1) }\end{array}$ & \\
\hline $4 q 23-q 27$ & AGA & C & Aspartylglucosaminidase & 208400 & S, F, D & Aspartylglucosaminuria (3) & \\
\hline $4 q 23-q 27$ & RGS & $\mathbf{P}$ & Rieger syndrome & 180500 & $\mathrm{Ch}, \mathrm{Fd}$ & Rieger syndrome (2) & \\
\hline $4 \mathrm{q} 25$ & IF & C & $\begin{array}{l}\text { Complement component I (C3b } \\
\text { inactivator) }\end{array}$ & 217030 & $\begin{array}{l}\mathrm{REa}, \mathrm{Fd}, \mathrm{A}, \\
\mathrm{RE}\end{array}$ & $\mathrm{C} 3 \mathrm{~b}$ inactivator deficiency (1) & \\
\hline $4 q 26-q 27$ & IL2, TCGF & C & T-cell growth factor (interleukin-2) & 147680 & $\mathrm{REa}, \mathrm{A}, \mathrm{F}$ & $\begin{array}{l}\text { Severe combined } \\
\text { immunodeficiency due to IL2 } \\
\text { deficiency (1) }\end{array}$ & $3(\mathrm{Il}-2)$ \\
\hline $4 q 28$ & FGA & $\mathrm{C}$ & Fibrinogen, alpha chain & 134820 & $\begin{array}{l}\text { RE, REa, H, } \\
\text { D, LD, A }\end{array}$ & Dysfibrinogenemia, alpha types (1) & \\
\hline $4 q 28$ & FGB & $\mathrm{C}$ & Fibrinogen, beta chain & 134830 & $\begin{array}{l}\text { RE, REa, D, } \\
\text { LD, A }\end{array}$ & Dysfibrinogenemia, beta types (1) & \\
\hline $4 q 28$ & FGG & $\mathrm{C}$ & Fibrinogen, gamma chain & 134850 & $\begin{array}{l}\text { F, REa, H, } \\
\text { RE, D, LD, } \\
\text { A }\end{array}$ & $\begin{array}{l}\text { Dysfibrinogenemia, gamma types } \\
\text { (1); Hypofibrinogenemia, gamma } \\
\text { types (1) }\end{array}$ & 3(Fgg) \\
\hline $4 q 28-q 31$ & ASMD & $\mathbf{P}$ & $\begin{array}{l}\text { Anterior segment mesenchymal } \\
\text { dysgenesis }\end{array}$ & 107250 & $\mathbf{F}$ & $\begin{array}{l}\text { Anterior segment mesenchymal } \\
\text { dysgenesis }(2)\end{array}$ & \\
\hline $4 q 28-q 31$ & TYS & C & Sclerotylosis & 181600 & $\mathbf{F}$ & Sclerotylosis (2) & \\
\hline $4 \mathrm{q} 31.1$ & $\begin{array}{l}\text { MLR, MCR, } \\
\text { MR }\end{array}$ & $\mathrm{C}$ & Mineralocorticoid receptor & 264350 & REa, M, A & Pseudohypoaldosteronism (1) & \\
\hline $4 \mathrm{q} 32.1$ & $\begin{array}{l}\text { HVBS6, } \\
\text { HCC2 }\end{array}$ & $\mathbf{P}$ & $\begin{array}{l}\text { Hepatitis B virus integration site- } 6 \\
\text { (hepatocellular carcinoma-2) }\end{array}$ & 142380 & $\mathrm{REa}, \mathrm{A}, \mathrm{D}$ & Hepatocellular carcinoma (3) & \\
\hline $4 \mathrm{q} 35$ & F11 & C & Coagulation factor XI & 264900 & $A, H, F d$ & Factor XI deficiency (1) & $8(\mathrm{cf}-11)$ \\
\hline $4 q 35$ & FMD, FSHD & C & $\begin{array}{l}\text { Facioscapulohumeral muscular } \\
\text { dystrophy }\end{array}$ & 158900 & $\mathrm{Fd}$ & $\begin{array}{l}\text { Facioscapulohumeral muscular } \\
\text { dystrophy (2) }\end{array}$ & \\
\hline $4 \mathrm{q} 35$ & KLK3 & $\mathbf{P}$ & Kallikrein, plasma & 229000 & A & Fletcher factor deficiency (1) & $8(\mathrm{Kal}-3)$ \\
\hline $5 p 13$ & $\mathrm{C} 6$ & $\mathrm{C}$ & Complement component- 6 & 217050 & $\begin{array}{l}\text { A, H, RE, Fd, } \\
\text { LD }\end{array}$ & $\begin{array}{l}\text { C6 deficiency (1); Combined C6/C7 } \\
\text { deficiency (1) }\end{array}$ & $15(\mathrm{C} 6)$ \\
\hline $5 \mathrm{p} 13$ & $\mathrm{C} 7$ & C & Complement component-7 & 217070 & $\begin{array}{l}A, H, R E, F d, \\
\text { LD }\end{array}$ & C7 deficiency (1) & $15(C 7)$ \\
\hline
\end{tabular}


Table 1-contd

\begin{tabular}{|c|c|c|c|c|c|c|c|}
\hline Location & Symbol & Status & Title & MIM\# & Method & Disorder & Mouse \\
\hline $5 \mathrm{p} 13$ & $\mathrm{C} 9$ & $\mathrm{C}$ & Complement component-9 & 120940 & $\begin{array}{l}\text { REa, A, Fd, } \\
\text { LD }\end{array}$ & C9 deficiency (1) & \\
\hline 5p13-p12 & GHR, GHBP & C & Growth hormone receptor & 262500 & $\mathrm{REa}, \mathrm{A}$ & Laron dwarfism (1) & $15(\mathrm{Ghr})$ \\
\hline 5p13-p12 & BBBG & L & $\begin{array}{l}\text { Hypospadias-dysphagia syndrome } \\
\text { (Opitz BBBG syndrome) }\end{array}$ & 145410 & $\mathrm{Ch}$ & $\begin{array}{l}\text { ?Hypospadias-dysphagia syndrome } \\
\text { (2) }\end{array}$ & \\
\hline $5 q 11-q 13$ & ARSB & C & Arylsulfatase B & 253200 & $\mathbf{S}$ & $\begin{array}{l}\text { Maroteaux-Lamy syndrome, } \\
\text { several forms (1) }\end{array}$ & 13(As-1) \\
\hline $5 q 11.2-q 13.2$ & DHFR & C & Dihydrofolate reductase & 126060 & S, REa, H, D & $\begin{array}{l}\text { ?Anemia, megaloblastic, due to } \\
\text { DHFR deficiency (1) }\end{array}$ & 13(Dhfr) \\
\hline $5 q 11.2-q 13.3$ & SCZD1 & $\mathbf{L}$ & Schizophrenia-1 & 181510 & $\mathrm{Ch}, \mathrm{Fd}$ & ?Schizophrenia (2) & \\
\hline $5 q 12-q 32$ & MAR & $\mathbf{P}$ & Macrocytic anemia, refractory & 153550 & $\mathrm{Ch}$ & $\begin{array}{l}\text { Macrocytic anemia of } 5 \mathrm{q}- \\
\text { syndrome, refractory }(2)\end{array}$ & \\
\hline $5 q 12.2-q 13.3$ & SMA & $\mathrm{C}$ & Spinal muscular atrophy & 253300 & Fd & $\begin{array}{l}\text { Werdnig-Hoffmann disease (2); } \\
\text { Spinal muscular atrophy II (2); } \\
\text { Spinal muscular atrophy III (2) }\end{array}$ & \\
\hline $5 q 13$ & HEXB & $\mathrm{C}$ & $\begin{array}{l}\text { Hexosaminidase B (beta } \\
\text { polypeptide) }\end{array}$ & 268800 & $\mathrm{~S}, \mathrm{Ch}, \mathrm{D}$ & Sandhoff disease (1) & 13(Hex-2) \\
\hline $5 q 21$ & MCC & C & Mutated in colorectal cancers & 159350 & REn, D & Colorectal cancer (1) & $18(\mathrm{Mcc})$ \\
\hline $5 q 21-q 22$ & $\begin{array}{l}\text { APC, GS, } \\
\text { FPC }\end{array}$ & $\mathrm{C}$ & $\begin{array}{l}\text { Adenomatous polyposis of the } \\
\text { colon (Gardner syndrome; } \\
\text { familial polyposis coli) }\end{array}$ & 175100 & D, Fd, REn & $\begin{array}{l}\text { Gardner syndrome (3); Polyposis } \\
\text { coli, familial (3); Colorectal } \\
\text { cancer (3) }\end{array}$ & 18(Min) \\
\hline $5 q 22.3-q 31.3$ & LGMD1 & $\mathbf{P}$ & $\begin{array}{l}\text { Limb-girdle muscular dystrophy, } \\
\text { autosomal dominant }\end{array}$ & 159000 & Fd & $\begin{array}{l}\text { Muscular dystrophy, limb-girdle, } \\
\text { autosomal dominant (2) }\end{array}$ & \\
\hline $5 q 23$ & DTS & $\mathrm{C}$ & Diphtheria toxin sensitivity & 126150 & $\mathrm{~S}, \mathrm{M}$ & $\{$ Diphtheria, susceptibility to\} (1) & \\
\hline $5 q 31$ & GRL & $\mathrm{C}$ & $\begin{array}{l}\text { Glucocorticoid receptor, } \\
\text { lymphocyte }\end{array}$ & 138040 & $\begin{array}{l}\text { S, REa, Fd, } \\
\text { H, A, D, } \\
\text { REn }\end{array}$ & Cortisol resistance $(1)$ & $18(\mathrm{Grl}-1)$ \\
\hline $5 q 31-q 33$ & LFHL1 & $\mathbf{P}$ & Progressive low-frequency deafness & 124900 & Fd & Deafness, low-tone (2) & \\
\hline $5 q 31-q 34$ & DTD, DD & $\mathbf{P}$ & Diastrophic dysplasia & 222600 & Fd & Diastrophic dysplasia (2) & \\
\hline $5 q 31.3-q 33.3$ & $\begin{array}{l}\text { TCOF1, } \\
\text { TCS, } \\
\text { MFD1 }\end{array}$ & C & $\begin{array}{l}\text { Treacher Collins mandibulofacial } \\
\text { dysostosis }\end{array}$ & 154500 & $\mathrm{Ch}, \mathrm{Fd}$ & $\begin{array}{l}\text { Treacher Collins mandibulofacial } \\
\text { dysostosis }(2)\end{array}$ & \\
\hline $5 q 33-q 35$ & STHE & $\mathbf{P}$ & $\begin{array}{l}\text { Startle disease/hyperekplexia (stiff } \\
\text { man syndrome) }\end{array}$ & 149400 & Fd & Startle disease (2) & \\
\hline 5q33-qter & F12, HAF & C & $\begin{array}{l}\text { Coagulation factor XII (Hageman } \\
\text { factor) }\end{array}$ & 234000 & REa, A & Factor XII deficiency (1) & \\
\hline Chr.5 & FBN2, CCA & $\mathbf{P}$ & Fibrillin-2 & 121050 & $\mathrm{REa}, \mathrm{Fd}$ & $\begin{array}{l}\text { Contractural arachnodactyly, } \\
\text { congenital ( } 3 \text { ) }\end{array}$ & \\
\hline Chr.5 & GM2A & C & GM2-activator protein & 272750 & S, REa & GM2-gangliosidosis, AB variant (1) & \\
\hline 6pter-p23 & OFC, CL & $\mathbf{P}$ & $\begin{array}{l}\text { Orofacial cleft (cleft lip with or } \\
\text { without cleft palate; isolated cleft } \\
\text { palate) }\end{array}$ & 119530 & $\mathrm{~F}$ & Orofacial cleft $(2)$ & \\
\hline 6p25-p24 & F13A1, F13A & C & $\begin{array}{l}\text { Coagulation factor XIII, A } \\
\text { polypeptide }\end{array}$ & 134570 & F, Fd, A, D & Factor XIIIA deficiency (3) & \\
\hline $6 p 22-p 21$ & $\begin{array}{l}\text { BCKDHB, } \\
\text { E1B }\end{array}$ & C & $\begin{array}{l}\text { Branched-chain keto acid } \\
\text { dehydrogenase E1, beta } \\
\text { polypeptide }\end{array}$ & 248611 & $\mathrm{REa}, \mathrm{A}$ & $\begin{array}{l}\text { Maple syrup urine disease, type } 3 \\
\text { (1) }\end{array}$ & \\
\hline $6 \mathrm{p} 21.3$ & ASD2 & $\mathbf{P}$ & Atrial septal defect, secundum type & 108800 & $\mathbf{F}$ & $\begin{array}{l}\text { Atrial septal defect, secundum type } \\
\text { (2) }\end{array}$ & \\
\hline $6 \mathrm{p} 21.3$ & C2 & C & Complement component-2 & 217000 & F, LD, RE & C2 deficiency (3) & $17(\mathrm{C} 2)$ \\
\hline $6 \mathrm{p} 21.3$ & $\mathrm{C} 4 \mathrm{~A}, \mathrm{C} 4 \mathrm{~S}$ & C & Complement component-4A & 120810 & $\mathrm{~F}, \mathrm{H}, \mathrm{RE}, \mathrm{Fd}$ & C4 deficiency (3) & $17(\mathrm{C} 4)$ \\
\hline $6 \mathrm{p} 21.3$ & $\mathrm{C} 4 \mathrm{~B}, \mathrm{C} 4 \mathrm{~F}$ & C & Complement component-4B & 120820 & $\mathrm{~F}, \mathrm{H}, \mathrm{RE}, \mathrm{Fd}$ & C4 deficiency ( 3 ) & $17(\mathrm{C} 4)$ \\
\hline $6 \mathrm{p} 21.3$ & $\begin{array}{l}\text { CYP21, } \\
\text { CA21H, } \\
\text { CAH1 }\end{array}$ & C & $\begin{array}{l}\text { Cytochrome } \mathrm{P450} \text {, subfamily XXI; } \\
\text { steroid } 21 \text {-hydroxylase, } \\
\text { congenital adrenal hyperplasia }\end{array}$ & 201910 & F, RE & $\begin{array}{l}\text { Adrenal hyperplasia, congenital, } \\
\text { due to } 21 \text {-hydroxylase deficiency } \\
\text { (3) }\end{array}$ & 17(Сур21) \\
\hline $6 \mathrm{p} 21.3$ & EJM1, JME & C & Epilepsy, juvenile myoclonic & 254770 & $\mathrm{~F}, \mathrm{Fd}$ & Epilepsy, juvenile myoclonic (2) & \\
\hline $6 \mathrm{p} 21.3$ & GLUR & $\mathbf{P}$ & Renal glucosuria & 233100 & $\mathbf{F}$ & [Renal glucosuria] (2) & \\
\hline $6 \mathrm{p} 21.3$ & HFE & C & Hemochromatosis & 235200 & $\mathrm{LD}, \mathrm{F}$ & Hemochromatosis (2) & \\
\hline $6 \mathrm{p} 21.3$ & IDDM & $\mathrm{L}$ & Insulin dependent diabetes mellitus & 222100 & F, LD & $\begin{array}{l}\text { ?Diabetes mellitus, } \\
\text { insulin-dependent (2) }\end{array}$ & \\
\hline $6 \mathrm{p} 21.3$ & NDF & L & Neutrophil differentiation factor & 202700 & LD & ?Kostmann agranulocytosis (2) & \\
\hline $6 \mathrm{p} 21.3$ & NEU, NEU1 & I & Neuraminidase- 1 ; sialidosis & 256550 & $\mathbf{H}, \mathbf{F}$ & ?Sialidosis (2) & $17(\mathrm{Neu}-1)$ \\
\hline $6 \mathrm{p} 21.3$ & PDB & L & Paget disease of bone & 167250 & $\mathbf{F}$ & ?Paget disease of bone (2) & \\
\hline $6 \mathrm{p} 21.3$ & RWS & $\mathbf{L}$ & Ragweed sensitivity & 179450 & $\mathbf{F}$ & ?Ragweed sensitivity (2) & \\
\hline $6 \mathrm{p} 21.3-\mathrm{p} 21.2$ & LAP & $\mathbf{L}$ & Laryngeal adductor paralysis & 150270 & $\mathbf{F}$ & ?Laryngeal adductor paralysis (2) & \\
\hline $6 \mathrm{p} 21.3-\mathrm{p} 21.2$ & SCA1 & C & Spinocerebellar ataxia-1 & 164400 & $\mathrm{~F}, \mathrm{Fd}$ & Spinocerebellar ataxia-1 (2) & \\
\hline 6p21.1-cen & RDS & C & $\begin{array}{l}\text { Retinal degeneration, slow } \\
\text { (peripherin) }\end{array}$ & 179605 & REa, A & $\begin{array}{l}\text { Retinitis pigmentosa, } \\
\text { peripherin-related (3) }\end{array}$ & $17(\mathrm{rds})$ \\
\hline $6 \mathrm{p} 21$ & MUT, MCM & C & Methylmalonyl CoA mutase & 251000 & REa, A, F, D & $\begin{array}{l}\text { Methylmalonicaciduria, mutase } \\
\text { deficiency type (1) }\end{array}$ & 17(Mut) \\
\hline $6 \mathrm{p}$ & ICS & $\mathbf{L}$ & Immotile cilia syndrome & 242650 & $\mathbf{F}$ & ?immotile cilia syndrome (2) & \\
\hline 6q13-q15 & OAR & $\mathbf{L}$ & $\begin{array}{l}\text { Ocular albinism, autosomal } \\
\text { recessive }\end{array}$ & 203310 & $\mathrm{Ch}$ & $\begin{array}{l}\text { ?Ocular albinism autosomal } \\
\text { recessive }(2)\end{array}$ & \\
\hline $6 q 13-q 21$ & $\begin{array}{l}\text { MCDR1, } \\
\text { NCMD }\end{array}$ & $\mathbf{P}$ & $\begin{array}{l}\text { Macular dystrophy, retinal, } 1 \\
\text { (North Carolina type) }\end{array}$ & 136550 & Fd & $\begin{array}{l}\text { Macular dystrophy, North Carolina } \\
\text { type (2) }\end{array}$ & \\
\hline $6 q 23$ & ARG1 & $\mathbf{P}$ & Arginase, liver & 207800 & REa & Argininemia (1) & \\
\hline $6 \mathrm{q} 24-\mathrm{q} 27$ & ESR, ER & C & Estrogen receptor & 133430 & REa, A & Breast cancer $(1)$ & \\
\hline $6 q 25-q 26$ & RCD1 & L & Retinal cone dystrophy-1 & 180020 & $\mathrm{Ch}$ & ?Retinal cone dystrophy-1 (2) & \\
\hline $6 \mathrm{q} 26-\mathrm{q} 27$ & PLG & $\mathrm{C}$ & Plasminogen & 173350 & $\begin{array}{l}\text { REa, A, LD, } \\
\text { F }\end{array}$ & $\begin{array}{l}\text { Plasminogen Tochigi disease (1); } \\
\text { Dysplasminogenemic } \\
\text { thrombophilia (1); Plasminogen } \\
\text { deficiency, types I and II (1) }\end{array}$ & 17(Plg) \\
\hline $6 \mathrm{q} 27$ & LPA & C & Apolipoprotein $\mathrm{Lp}(\mathrm{a})$ & 152200 & REa, A, F, Fd & $\begin{array}{l}\text { \{Coronary artery disease, } \\
\text { susceptibility to (1) }\end{array}$ & \\
\hline $7 \mathrm{p} 21.3-\mathrm{p} 21.2$ & CRS, CSO & $\mathrm{C}$ & Craniosynostosis & 123100 & $\mathrm{Ch}$ & Craniosynostosis (2) & \\
\hline 7p15-p13 & GCK & $\mathbf{P}$ & Glucokinase & 138079 & Psh, Fd & MODY, type II, 125851 (3) & \\
\hline $7 \mathrm{p} 13$ & GCPS & $\mathrm{C}$ & $\begin{array}{l}\text { Greig craniopolysyndactyly } \\
\text { syndrome }\end{array}$ & 175700 & $\begin{array}{l}\mathrm{Ch}, \mathrm{Fd}, \mathrm{REn}, \\
\mathrm{D}\end{array}$ & $\begin{array}{l}\text { Greig craniopolysyndactyly } \\
\text { syndrome (3) }\end{array}$ & $13(\mathrm{Xt})$ \\
\hline
\end{tabular}


Table 1-contd

\begin{tabular}{|c|c|c|c|c|c|c|c|}
\hline Location & Symbol & Status & Title & MIM\# & Method & Disorder & Mouse \\
\hline 7p13-p12.3 & $\begin{array}{l}\text { PGAM2, } \\
\text { PGAMM }\end{array}$ & $\mathrm{C}$ & $\begin{array}{l}\text { Phosphoglycerate mutase, muscle } \\
\text { form }\end{array}$ & 261670 & REa, A & $\begin{array}{l}\text { Myopathy due to phosphoglycerate } \\
\text { mutase deficiency (1) }\end{array}$ & \\
\hline $7 \mathrm{p}$ & GHS & $\mathrm{L}$ & Goldenhar syndrome & 141400 & $\mathrm{Ch}$ & ?Goldenhar syndrome (2) & \\
\hline 7 cen-q11.2 & ASL & $\mathrm{C}$ & Argininosuccinate lyase & 207900 & $\mathrm{~S}, \mathrm{REa}, \mathrm{A}$ & Argininosuccinicaciduria (1) & 5 (Asl) \\
\hline $7 \mathrm{q} 11.2-\mathrm{q} 21.3$ & EEC & $\mathrm{L}$ & $\begin{array}{l}\text { Ectrodactyly, ectodermal dysplasia, } \\
\text { cleft lip/palate }\end{array}$ & 129900 & $\mathrm{Ch}$ & ?EEC syndrome (2) & \\
\hline $7 q 11.23$ & NCF1 & $P$ & Neutrophil cytosolic factor-1 & 233700 & REa, A & $\begin{array}{l}\text { Chronic granulomatous disease due } \\
\text { to deficiency of NCF-1 (1) }\end{array}$ & \\
\hline $7 q 11.23$ & $\begin{array}{l}\text { ZWS1, ZWS, } \\
\text { ZS }\end{array}$ & $\mathrm{C}$ & Zellweger syndrome-1 & 214100 & $\mathrm{Ch}$ & Zellweger syndrome-1 (2) & \\
\hline $7 \mathrm{q} 21$ & EPO & $\mathrm{C}$ & Erythropoietin & 133170 & $\begin{array}{l}\text { REa, } A, R E b \text {, } \\
\text { Fd }\end{array}$ & ?Erythremia (1) & 5(Epo) \\
\hline $7 \mathrm{q} 21.11$ & GUSB & $\mathrm{C}$ & Beta-glucuronidase & 253220 & $\mathrm{~S}, \mathrm{D}, \mathrm{EM}$ & Mucopolysaccharidosis VII (1) & 5 (Gus) \\
\hline $7 \mathrm{q} 21.2-\mathrm{q} 21.3$ & SHFD1 & $\mathrm{C}$ & Split-hand/foot deformity, type 1 & 183600 & $\mathrm{Ch}$ & $\begin{array}{l}\text { Split-hand/foot deformity, type } 1 \\
\text { (2) }\end{array}$ & \\
\hline $7 \mathrm{q} 21.3-\mathrm{q} 22$ & $\begin{array}{l}\text { PLANH1, } \\
\text { PAI1 }\end{array}$ & $\mathrm{C}$ & $\begin{array}{l}\text { Plasminogen activator inhibitor, } \\
\text { type I }\end{array}$ & 173360 & $\begin{array}{l}\text { REa, REb, } \\
\text { Fd, A, D }\end{array}$ & $\begin{array}{l}\text { Thrombophilia due to excessive } \\
\text { plasminogen activator inhibitor } \\
\text { (1); Hemorrhagic diathesis due to } \\
\text { PAIl deficiency (1) }\end{array}$ & \\
\hline $7 \mathrm{q} 21.3-\mathrm{q} 22.1$ & COL1A2 & $\mathrm{C}$ & Collagen I, alpha-2 polypeptide & 120160 & S, REa, D, A & $\begin{array}{l}\text { Osteogenesis imperfecta, } 2 \text { or more } \\
\text { clinical forms (3); Ehlers-Danlos } \\
\text { syndrome, type VIIA2 (3) }\end{array}$ & $6($ Cola-2) \\
\hline 7q22-qter & $\mathrm{BCP}, \mathrm{CBT}$ & $\mathbf{P}$ & Blue cone pigment & 190900 & REa & Colorblindness, tritan (2) & \\
\hline $7 \mathrm{q} 31$ & OB & $\mathrm{L}$ & Obesity & 164160 & $\mathrm{H}$ & ?Obesity (2) & $6(\mathrm{ob})$ \\
\hline $7 q 31-q 32$ & $\begin{array}{l}\text { DLD, LAD, } \\
\text { PHE3 }\end{array}$ & $\mathrm{C}$ & $\begin{array}{l}\text { Dihydrolipoamide dehydrogenase } \\
\text { (pyruvate dehydrogenase } \\
\text { component E3) }\end{array}$ & 246900 & REa & $\begin{array}{l}\text { Lipoamide dehydrogenase } \\
\text { deficiency (1) }\end{array}$ & \\
\hline $7 q 31-q 34$ & BPGM & $\mathbf{P}$ & 2,3-bisphosphoglycerate mutase & 222800 & A & $\begin{array}{l}\text { Hemolytic anemia due to } \\
\text { bisphosphoglycerate mutase } \\
\text { deficiency (1) }\end{array}$ & \\
\hline $7 \mathrm{q} 31.1-\mathrm{q} 31.3$ & LAMB1 & $\mathrm{C}$ & Laminin B1 & 150240 & $\mathrm{REa}, \mathrm{A}, \mathrm{Ch}$ & $\begin{array}{l}\text { ?Cutis laxa, marfanoid neonatal } \\
\text { type (1) }\end{array}$ & 1(Lamb-1) \\
\hline $7 q 31.2$ & CFTR, CF & $\mathrm{C}$ & $\begin{array}{l}\text { Cystic fibrosis transmembrane } \\
\text { conductance regulator }\end{array}$ & 219700 & $\mathrm{~F}, \mathrm{Fd}$ & $\begin{array}{l}\text { Cystic fibrosis (3); congenital } \\
\text { absence of vas deferens (1) }\end{array}$ & $6(\mathrm{Cftr})$ \\
\hline 7q32-qter & TRY1, TRP1 & $\mathbf{P}$ & Trypsin-1 & 276000 & REa & Trypsinogen deficiency (1) & $6($ Try -1$)$ \\
\hline 7q34-qter & SLO & $\mathrm{L}$ & Smith-Lemli-Opitz syndrome & 270400 & $\mathrm{Ch}$ & ?Smith-Lemli-Opitz syndrome (2) & \\
\hline $7 q 36$ & HLP3 & $\mathbf{P}$ & Holoprosencephaly, type 3 & 142945 & $\mathrm{Ch}$ & Holoprosencephaly, type 3 (2) & \\
\hline $7 q 36$ & HPFH2 & $\mathrm{L}$ & $\begin{array}{l}\text { Hereditary persistence of fetal } \\
\text { hemoglobin, heterocellular, } \\
\text { Indian type }\end{array}$ & 142335 & $\mathrm{Fd}$ & $\begin{array}{l}\text { ?Hereditary persistence of fetal } \\
\text { hemoglobin, heterocellular, } \\
\text { Indian type (2) }\end{array}$ & \\
\hline Chr.7 & HADH & $\mathbf{P}$ & Hydroxyacyl-CoA dehydrogenase & 143450 & $S$ & $\begin{array}{l}\text { 3-hydroxyacyl-CoA dehydrogenase } \\
\text { deficiency (1) }\end{array}$ & \\
\hline $8 \mathrm{p} 22$ & LPL, LIPD & $\mathbf{P}$ & Lipoprotein lipase (lipase D) & 238600 & $\mathrm{REa}, \mathrm{A}$ & Hyperlipoproteinemia I (1) & $8(\mathrm{Lpl})$ \\
\hline $8 \mathrm{p} 21.1$ & GSR & $\mathrm{C}$ & Glutathione reductase & 138300 & S, D & $\begin{array}{l}\text { Hemolytic anemia due to } \\
\text { glutathione reductase deficiency } \\
\text { (1) }\end{array}$ & $8(\mathrm{Gr}-1)$ \\
\hline $8 \mathrm{p} 21-\mathrm{p} 11.2$ & $\begin{array}{l}\text { LHRH, } \\
\text { GNRH }\end{array}$ & $\mathbf{P}$ & $\begin{array}{l}\text { Luteinizing hormone releasing } \\
\text { hormone (gonadotropin releasing } \\
\text { hormone) }\end{array}$ & 152760 & $\mathrm{REa}, \mathrm{A}$ & $\begin{array}{l}\text { ?Hypogonadotropic hypogonadism } \\
\text { due to GNRH deficiency, } 227200 \\
\text { (1) }\end{array}$ & $14(\mathrm{Gnrh})$ \\
\hline $8 \mathrm{p} 12$ & PLAT, TPA & $\mathrm{C}$ & Plasminogen activator, tissue type & 173370 & $\mathrm{REa}, \mathrm{A}, \mathrm{REb}$ & $\begin{array}{l}\text { Plasminogen activator deficiency } \\
\text { (1) }\end{array}$ & 8(Plat) \\
\hline $8 \mathrm{p} 12-\mathrm{p} 11$ & WRN & $\mathbf{P}$ & Werner syndrome & 277700 & $\mathrm{Fd}$ & Werner syndrome $(2)$ & \\
\hline $8 \mathrm{p} 11.2$ & ANK1, SPH2 & $\mathrm{C}$ & Ankyrin-1, erythrocytic & 182900 & $\begin{array}{l}\mathrm{F}, \mathrm{Ch}, \mathrm{D}, \\
\mathrm{REa}, \mathrm{A}, \mathrm{Fd}, \\
\mathrm{REb}\end{array}$ & Spherocytosis-2 (3) & $8(\mathrm{nb})$ \\
\hline $8 p 11-q 21$ & RP1 & $\mathbf{P}$ & Retinitis pigmentosa-1 & 180100 & Fd & Retinitis pigmentosa-1 (2) & \\
\hline $8 \mathrm{q} 12$ & SGPA, PSA & $\mathbf{P}$ & $\begin{array}{l}\text { Salivary gland pleomorphic } \\
\text { adenoma }\end{array}$ & 181030 & $\mathrm{Ch}$ & $\begin{array}{l}\text { Salivary gland pleomorphic } \\
\text { adenoma (2) }\end{array}$ & \\
\hline $8 \mathrm{q} 13.3$ & BOR, BOS & C & Branchiootic syndrome & 113650 & $\mathrm{Ch}, \mathrm{Fd}$ & Branchiootic syndrome (2) & \\
\hline $8 \mathrm{q} 21$ & $\begin{array}{l}\text { CYP11B1, } \\
\text { P450C11 }\end{array}$ & $\mathrm{C}$ & $\begin{array}{l}\text { Cytochrome P450, subfamily XIB, } \\
\text { polypeptide-1; } \\
\text { 11-beta-hydroxylase; } \\
\text { corticosteroid methyl-oxidase II } \\
\text { (CMO II) }\end{array}$ & 202010 & $\mathrm{REa}, \mathrm{A}, \mathrm{Ch}$ & $\begin{array}{l}\text { Adrenal hyperplasia, congenital, } \\
\text { due to 11-beta-hydroxylase } \\
\text { deficiency (1); CMO II } \\
\text { deficiency (1) }\end{array}$ & \\
\hline $8 \mathrm{q} 21$ & CYP11B2 & C & $\begin{array}{l}\text { Cytochrome P450, subfamily XIB, } \\
\text { polypeptide- } 2\end{array}$ & 124080 & REa & CMO II deficiency (1) & \\
\hline $8 \mathrm{q} 21.4$ & CMD1 & $\mathrm{L}$ & $\begin{array}{l}\text { Campomelic dysplasia with sex } \\
\text { reversal }\end{array}$ & 211970 & $\mathrm{Ch}$ & $\begin{array}{l}\text { ?Campomelic dysplasia with sex } \\
\text { reversal (2) }\end{array}$ & \\
\hline $8 \mathrm{q} 22$ & CA1 & $\mathrm{C}$ & Carbonic anhydrase I & 114800 & $\mathrm{REa}, \mathrm{H}, \mathrm{A}$ & $\begin{array}{l}\text { [Carbonic anhydrase I deficiency] } \\
\text { (1) }\end{array}$ & $3(\mathrm{Car}-1)$ \\
\hline $8 \mathrm{q} 22$ & CA2 & C & Carbonic anhydrase II & 259730 & $\mathrm{REa}, \mathrm{H}$ & $\begin{array}{l}\text { Renal tubular acidosis-osteopetrosis } \\
\text { syndrome (1) }\end{array}$ & $3($ Car-2) \\
\hline $8 \mathrm{q} 22$ & CLCD & $\mathrm{L}$ & Cleidocranial dysplasia & 119600 & $\mathrm{Ch}$ & ?Cleidocranial dysplasia (2) & \\
\hline $8 \mathrm{q} 23-\mathrm{q} 24.1$ & EXT & $\mathrm{L}$ & Multiple exostoses & 133700 & $\mathrm{Ch}$ & ?Multiple exostoses (2) & \\
\hline $8 \mathrm{q} 24$ & EBS1 & $\mathrm{C}$ & $\begin{array}{l}\text { Epidermolysis bullosa simplex-1 } \\
\text { (Ogna) }\end{array}$ & 131950 & $\mathbf{F}$ & $\begin{array}{l}\text { Epidermolysis bullosa, Ogna type } \\
\text { (2) }\end{array}$ & \\
\hline $8 q 24$ & PDS & $\mathbf{L}$ & Pendred syndrome & 274600 & $\mathrm{Ch}$ & ?Pendred syndrome (2) & \\
\hline $8 \mathrm{q} 24$ & VMD1 & $\bar{C}$ & $\begin{array}{l}\text { Macular dystrophy, atypical } \\
\text { vitelliform }\end{array}$ & 153840 & $\mathrm{~F}$ & $\begin{array}{l}\text { Macular dystrophy, atypical } \\
\text { vitelliform (2) }\end{array}$ & \\
\hline $8 \mathrm{q} 24.11-\mathrm{q} 24.13$ & $\begin{array}{l}\text { LGCR, LGS, } \\
\text { TRPS2 }\end{array}$ & C & Langer-Giedion syndrome & 150230 & $\mathrm{Ch}$ & Langer-Giedion syndrome (2) & \\
\hline $8 \mathrm{q} 24.12$ & TRPS 1 & $\mathbf{P}$ & $\begin{array}{l}\text { Trichorhinophalangeal syndrome, } \\
\text { type I }\end{array}$ & 190350 & $\mathrm{Ch}$ & $\begin{array}{l}\text { Trichorhinophalangeal syndrome, } \\
\text { type I (2) }\end{array}$ & \\
\hline $8 \mathrm{q} 24.12-\mathrm{q} 24.13$ & MYC & $\mathrm{C}$ & $\begin{array}{l}\text { Oncogene MYC, avian } \\
\text { myelocytomatosis virus }\end{array}$ & 190080 & $\mathrm{REa}, \mathrm{A}$ & Burkitt lymphoma (3) & $15(\mathrm{Myc})$ \\
\hline $8 \mathrm{q} 24.2-\mathrm{q} 24.3$ & TG & C & Thyroglobulin & 188450 & A, REa, REb & $\begin{array}{l}\text { Hypothyroidism, hereditary } \\
\text { congenital (1); Goiter, adolescent } \\
\text { multinodular (1) }\end{array}$ & 15(Tgn; cog) \\
\hline
\end{tabular}


Table 1-contd

\begin{tabular}{|c|c|c|c|c|c|c|c|}
\hline Location & Symbol & Status & Title & MIM\# & Method & Disorder & Mouse \\
\hline Chr.8 & RTS & $\mathrm{L}$ & Rothmund-Thomson syndrome & 268400 & $\mathrm{Ch}$ & ?Rothmund-Thomson syndrome (2) & \\
\hline $9 \mathrm{p} 24$ & OVC & $\mathbf{P}$ & $\begin{array}{l}\text { Oncogene OVC (ovarian } \\
\text { adenocarcinoma oncogene) }\end{array}$ & 164759 & $\mathrm{Ch}$ & Ovarian carcinoma $(2)$ & \\
\hline $9 \mathrm{p} 23$ & $\begin{array}{l}\text { TYRP, } \\
\text { CAS2, } \\
\text { CATB }\end{array}$ & C & $\begin{array}{l}\text { Tyrosinase-related protein (catalase } \\
\text { B; human homolog of 'brown" } \\
\text { locus) }\end{array}$ & 115501 & Psh, REa, A & ?Melanoma (1) & $4(b ; t r p-1)$ \\
\hline $9 \mathrm{p} 22$ & NKH1 & L & $\begin{array}{l}\text { Hyperglycinemia, isolated } \\
\text { nonketotic, type I }\end{array}$ & 238300 & $\mathrm{Ch}$ & $\begin{array}{l}\text { ?Hyperglycinemia, isolated } \\
\text { nonketotic, type I (2) }\end{array}$ & \\
\hline $9 \mathrm{p} 22-\mathrm{p} 21$ & LALL & $\mathbf{P}$ & $\begin{array}{l}\text { Lymphomatous acute } \\
\text { lymphoblastic leukemia }\end{array}$ & 247640 & $\mathrm{Ch}$ & Leukemia, acute lymphoblastic (2) & \\
\hline $9 \mathrm{p} 21$ & $\begin{array}{l}\text { IFNA, } \\
\text { IFL,IFA }\end{array}$ & C & Interferon, alpha (leukocyte) & 147660 & $\mathrm{REa}, \mathrm{A}, \mathrm{RE}$ & Interferon, alpha, deficiency (1) & $4($ Ifa) \\
\hline $9 \mathrm{p} 13$ & GALT & C & $\begin{array}{l}\text { Galactose-1-phosphate } \\
\text { uridyltransferase }\end{array}$ & 230400 & $S, D, F$ & Galactosemia (1) & 4(Galt) \\
\hline 9q13-q21.1 & FRDA, FAT & C & Friedreich ataxia & 229300 & $\mathrm{Fd}$ & Friedreich ataxia (2) & \\
\hline $9 \mathrm{q} 22$ & ALDOB & C & $\begin{array}{l}\text { Aldolase } \mathrm{B} \text {, } \\
\text { fructose-bisphosphatase }\end{array}$ & 229600 & $\begin{array}{l}\text { REb, REa, A, } \\
\text { D }\end{array}$ & Fructose intolerance (1) & \\
\hline $9 q 31$ & ESS1, MSSE & $\mathrm{P}$ & $\begin{array}{l}\text { Epithelioma, self-healing, } \\
\text { squamous 1, Ferguson-Smith } \\
\text { type }\end{array}$ & 132800 & $\mathrm{Fd}$ & $\begin{array}{l}\text { Epithelioma, self-healing, } \\
\text { squamous } 1 \text {, Ferguson-Smith } \\
\text { type (2) }\end{array}$ & \\
\hline $9 \mathrm{q} 31$ & $\begin{array}{l}\text { BCNS, } \\
\text { NBCCS }\end{array}$ & C & $\begin{array}{l}\text { Basal cell nevus syndrome (Gorlin } \\
\text { syndrome) }\end{array}$ & 109400 & Fd, D & Basal cell nevus syndrome (2) & \\
\hline $9 \mathrm{q} 31$ & TAL2 & $\mathbf{P}$ & $\begin{array}{l}\mathrm{T} \text {-cell acute lymphoblastic } \\
\text { leukemia-2 }\end{array}$ & 186855 & $\begin{array}{l}\text { REa, A, RE, } \\
\text { Ch }\end{array}$ & $\begin{array}{l}\text { Leukemia-2, T-cell acute } \\
\text { lymphoblastic }(3)\end{array}$ & \\
\hline $9 q 32-q 33$ & $\begin{array}{l}\text { AMBP, } \\
\text { ITIL, ITI, } \\
\text { HCP }\end{array}$ & C & $\begin{array}{l}\text { Alpha-1-microglobulin/bikunin; } \\
\text { inter-alpha-trypsin inhibitor, } \\
\text { light chain; protein HC }\end{array}$ & 176870 & $\mathrm{REa}, \mathrm{A}, \mathrm{H}$ & $\begin{array}{l}\text { ?Familial Mediterranean fever, } \\
249100 \text { (1) }\end{array}$ & 4(Intin-4) \\
\hline $9 q 32-q 34$ & DYT1 & $\mathrm{C}$ & $\begin{array}{l}\text { Torsion dystonia, autosomal } \\
\text { dominant }\end{array}$ & 128100 & Fd & Torsion dystonia (2) & \\
\hline $9 q 33-q 34$ & $\begin{array}{l}\text { TSC1, TSC, } \\
\text { TS }\end{array}$ & $\mathrm{C}$ & Tuberous sclerosis- 1 & 191100 & $\mathrm{~F}, \mathrm{Fd}$ & Tuberous sclerosis-1 (2) & \\
\hline 9q33-qter & ITO & I & Hypomelanosis of Ito & 146150 & $\mathrm{X} / \mathrm{A}$ & ?Hypomelanosis of Ito (2) & \\
\hline $9 q 34$ & ALAD & C & Delta-aminolevulinate dehydratase & 125270 & F, S, A, REa & $\begin{array}{l}\text { Porphyria, acute hepatic (1); Lead } \\
\text { poisoning, susceptibility to (1) }\end{array}$ & $4(\mathrm{Lv})$ \\
\hline 9q34 & ASS & $\mathrm{C}$ & Argininosuccinate synthetase & 215700 & $\begin{array}{l}\text { S, D, REa, } \\
\text { Fd }\end{array}$ & Citrullinemia (1) & 2 (Ass-1) \\
\hline $9 q 34$ & $\mathrm{DBH}$ & C & Dopamine-beta-hydroxylase & 223360 & F, A & $\begin{array}{l}\text { Autonomic failure due to DBH } \\
\text { deficiency (1) }\end{array}$ & $2(\mathrm{Dbh})$ \\
\hline $9 \mathrm{q} 34$ & GSN & $\mathbf{P}$ & Gelsolin & 137350 & A, REa, RE & $\begin{array}{l}\text { Amyloidosis, Finnish type, } 105120 \\
\text { (1) }\end{array}$ & $2(\mathrm{Gsn})$ \\
\hline $9 q 34$ & NPS1 & C & Nail-patella syndrome & 161200 & F, Fd & Nail-patella syndrome (2) & \\
\hline 9q34.1 & ABL & C & $\begin{array}{l}\text { Oncogene ABL (Abelson strain, } \\
\text { murine leukemia virus) }\end{array}$ & 189980 & $\mathrm{REa}, \mathrm{Ch}, \mathrm{A}$ & Leukemia, chronic myeloid ( 3 ) & $2(\mathrm{Abl})$ \\
\hline $9 q 34.1$ & $\mathrm{AK} 1$ & C & Adenylate kinase-1, soluble & 103000 & $\mathrm{~F}, \mathrm{~S}, \mathrm{D}, \mathrm{Fc}$ & $\begin{array}{l}\text { Hemolytic anemia due to adenylate } \\
\text { kinase deficiency (1) }\end{array}$ & $2(\mathrm{Ak}-1)$ \\
\hline $9 q 34.1$ & C5 & C & Complement component-5 & 120900 & $\mathrm{REa}, \mathrm{A}$ & C5 deficiency (1) & $2(\mathrm{Hc})$ \\
\hline 9q34.1 & XPA & C & $\begin{array}{l}\text { Xeroderma pigmentosum, } \\
\text { complementation group A }\end{array}$ & 278700 & S, A & $\begin{array}{l}\text { Xeroderma pigmentosum, type A } \\
\text { (1) }\end{array}$ & $4(\mathrm{Xpa})$ \\
\hline $9 \mathrm{q} 34.3$ & TAN1 & $\mathbf{P}$ & $\begin{array}{l}\text { Translocation-associated 'Notch' } \\
\text { homolog }\end{array}$ & 190198 & $\mathrm{Ch}$ & $\begin{array}{l}\text { Leukemia, T-cell acute } \\
\text { lymphoblastic }(2)\end{array}$ & \\
\hline Chr.9 & CPO, CPRO & $\mathbf{P}$ & Coproporphyrinogen oxidase & 121300 & D & $\begin{array}{l}\text { Coproporphyria (1); } \\
\text { Harderoporphyrinuria (1) }\end{array}$ & \\
\hline $\begin{array}{l}10 \mathrm{p} 12-\mathrm{q} 23.2 \\
10 \mathrm{q} 11.2-\mathrm{q} 21\end{array}$ & $\begin{array}{l}\text { GBM } \\
\text { MBL }\end{array}$ & $\begin{array}{l}\mathrm{C} \\
\mathrm{C}\end{array}$ & $\begin{array}{l}\text { Glioblastoma multiforme } \\
\text { Mannose-binding lectin }\end{array}$ & $\begin{array}{l}137800 \\
154545\end{array}$ & $\begin{array}{l}\text { D } \\
\text { REa, A, Fd }\end{array}$ & $\begin{array}{l}\text { Glioblastoma multiforme (2) } \\
\text { Chronic infections (1) }\end{array}$ & \\
\hline $10 \mathrm{q} 11-\mathrm{q} 12$ & $\begin{array}{l}\text { D10S170, } \\
\text { TST1, } \\
\text { PTC, TPC }\end{array}$ & $\mathrm{C}$ & $\begin{array}{l}\text { DNA segment, single copy, probe } \\
\text { pH4 (transforming sequence, } \\
\text { thyroid-1, from papillary thyroid } \\
\text { carcinoma) }\end{array}$ & 188550 & $\mathrm{REa}, \mathrm{A}$ & Thyroid papillary carcinoma (1) & \\
\hline $10 \mathrm{q} 21-\mathrm{q} 22$ & $\begin{array}{l}\text { PSAP, SAP1, } \\
\text { SAP2 }\end{array}$ & C & $\begin{array}{l}\text { Prosaposin (sphingolipid activator } \\
\text { protein-1; sphingolipid activator } \\
\text { protein-2) }\end{array}$ & 176801 & S, REa, A, D & $\begin{array}{l}\text { Metachromatic leukodystrophy due } \\
\text { to deficiency of SAP-1 (1); } \\
\text { Gaucher disease, variant form (1) }\end{array}$ & \\
\hline $10 \mathrm{q} 21.1$ & CS1 & $\mathbf{L}$ & $\begin{array}{l}\text { Cockayne syndrome, late-onset } \\
\text { form }\end{array}$ & 216400 & $\mathrm{Ch}$ & ?Cockayne syndrome $(2)$ & \\
\hline $10 \mathrm{q} 21.1$ & $\begin{array}{r}\text { MEN2A, } \\
\text { MEN2 }\end{array}$ & $\mathrm{C}$ & $\begin{array}{l}\text { Multiple endocrine neoplasia, type } \\
\text { II (or IIA); medullary thyroid } \\
\text { carcinoma }\end{array}$ & 171400 & $\mathrm{Fd}$ & $\begin{array}{l}\text { Multiple endocrine neoplasia II } \\
\text { (2); Medullary thyroid carcinoma } \\
\text { (2) }\end{array}$ & \\
\hline $10 \mathrm{q} 21.1$ & $\begin{array}{r}\text { MEN2B, } \\
\text { MEN3 }\end{array}$ & C & $\begin{array}{l}\text { Multiple endocrine neoplasia, type } \\
\text { IIB (or III) }\end{array}$ & 162300 & Fd & Multiple endocrine neoplasia III(2) & \\
\hline $10 \mathrm{q} 22$ & HK1 & C & Hexokinase-1 & 142600 & $\mathrm{~S}, \mathrm{D}, \mathrm{A}, \mathrm{REa}$ & $\begin{array}{l}\text { Hemolytic anemia due to } \\
\text { hexokinase deficiency (1) }\end{array}$ & $10(\mathrm{Hk}-1)$ \\
\hline $10 \mathrm{q} 23-\mathrm{q} 24$ & RBP4 & $\mathrm{C}$ & $\begin{array}{l}\text { Retinol-binding protein-4, } \\
\text { interstitial }\end{array}$ & 180250 & REa, A & $\begin{array}{l}\text { ?Retinol binding protein, deficiency } \\
\text { of (1) }\end{array}$ & 19(Rbp-4) \\
\hline 10q24 & $\begin{array}{r}\text { HOX11, } \\
\text { TCL3 }\end{array}$ & $\mathbf{P}$ & $\begin{array}{l}\text { Homeo box-11 ( } \mathrm{T} \text {-cell leukemia-3 } \\
\text { associated breakpoint) }\end{array}$ & 186770 & $\mathrm{Ch}$ & $\begin{array}{l}\text { Leukemia, T-cell acute } \\
\text { lymphocytic }(2)\end{array}$ & \\
\hline $10 q 24-q 25$ & LIPA & C & Lysosomal acid lipase-A & 278000 & $\mathrm{~S}, \mathrm{H}$ & $\begin{array}{l}\text { Wolman disease (1); Cholesteryl } \\
\text { ester storage disease (1) }\end{array}$ & 19(Lip-1) \\
\hline $10 \mathrm{q} 25.2-\mathrm{q} 26.3$ & UROS & $\mathbf{P}$ & Uroporphyrinogen III synthase & 263700 & REa, Psh & $\begin{array}{l}\text { Porphyria, congenital } \\
\text { erythropoietic (1) }\end{array}$ & \\
\hline $10 q 26$ & OAT & C & Ornithine aminotransferase & 258870 & S, REa, A, Fd & $\begin{array}{l}\text { Gyrate atrophy of choroid and } \\
\text { retina with ornithinemia, B6 } \\
\text { responsive or unresponsive (1) }\end{array}$ & 7 (Oat) \\
\hline $10 \mathrm{q} 26.1$ & PNLIP & $\mathbf{P}$ & Lipase, pancreatic & 246600 & $\mathrm{REa}, \mathrm{A}$ & Pancreatic lipase deficiency (1) & \\
\hline Chr.10 & $\begin{array}{l}\text { CYP17, } \\
\text { P450C17 }\end{array}$ & $\mathbf{P}$ & $\begin{array}{l}\text { Cytochrome P450, subfamily } \\
\text { XVII; steroid } \\
\text { 17-alpha-hydroxylase }\end{array}$ & 202110 & REa, $\mathbf{H}$ & Adrenal hyperplasia V (1) & 19(Cyp17) \\
\hline 11 pter-p15.4 & $\begin{array}{l}\text { BWS, } \\
\text { BWCR, } \\
\text { WBS }\end{array}$ & $\mathrm{C}$ & Beckwith-Wiedemann syndrome & 130650 & $\mathrm{Ch}, \mathrm{Fd}$ & $\begin{array}{l}\text { Beckwith-Wiedemann syndrome } \\
\text { (2) }\end{array}$ & \\
\hline
\end{tabular}


Table 1-contd

\begin{tabular}{|c|c|c|c|c|c|c|c|}
\hline Location & Symbol & Status & Title & MIM\# & Method & Disorder & Mouse \\
\hline 11 pter-p12 & SAA & C & Serum amyloid $\mathrm{A}$ & 104750 & $\mathrm{REa}, \mathrm{H}$ & $\begin{array}{l}\text { ?Susceptibility to amyloid in FMF, } \\
249100 \text { (1) }\end{array}$ & $7($ Saa-1) \\
\hline $11 \mathrm{p} 15.5$ & $\begin{array}{l}\text { ADCR, } \\
\text { ADCC }\end{array}$ & $\mathbf{P}$ & Adrenocortical carcinoma region & 202300 & D & Adrenocortical carcinoma (2) & \\
\hline $11 \mathrm{p} 15.5$ & $\mathrm{HBB}$ & C & Hemoglobin beta & 141900 & $\begin{array}{l}\text { LD, AAS, F, } \\
\text { Fd }\end{array}$ & $\begin{array}{l}\text { Sickle cell anemia (1); } \\
\text { Thalassemias, beta- (1); } \\
\text { Methemoglobinemias, beta- (1); } \\
\text { Erythremias, beta- (1); Heinz } \\
\text { body anemias, beta- (1); HPFH, } \\
\text { deletion type (1) }\end{array}$ & $7(\mathrm{Hbb})$ \\
\hline $11 \mathrm{p} 15.5$ & HBGR & $\mathrm{C}$ & Hb gamma regulator & 142270 & RE & $\begin{array}{l}\text { ?Hereditary persistence of fetal } \\
\text { hemoglobin ( } 3 \text { ) }\end{array}$ & \\
\hline $11 \mathrm{p} 15.5$ & HBG1 & C & Hemoglobin gamma 136 alanine & 142200 & RE & HPFH, nondeletion type A (1) & \\
\hline $11 \mathrm{p} 15.5$ & HBG2 & C & Hemoglobin gamma 136 glycine & 142250 & RE & HPFH, nondeletion type G (1) & \\
\hline $11 \mathrm{p} 15.5$ & INS & C & Insulin & 176730 & $\begin{array}{l}\text { HS, A, REb, } \\
\text { Fd, D }\end{array}$ & $\begin{array}{l}\text { Diabetes mellitus, rare form (1); } \\
\text { MODY, one form, } 125850 \text { (3); } \\
\text { Hyperproinsulinemia, familial (1) }\end{array}$ & $\begin{array}{l}\text { 6(Ins-1); } \\
\quad 7(\text { Ins-2) }\end{array}$ \\
\hline $11 \mathrm{p} 15.5$ & LQT & $\mathbf{P}$ & $\begin{array}{l}\text { Long QT syndrome } \\
\quad \text { (Romano-Ward syndrome) }\end{array}$ & 192500 & $\mathrm{Fd}$ & Long QT syndrome (2) & \\
\hline $11 \mathrm{p} 15.5$ & $\begin{array}{l}\text { MAFD1, } \\
\text { MD1 }\end{array}$ & $\mathrm{L}$ & $\begin{array}{l}\text { Manic-depressive illness (major } \\
\text { affective disorder } 1 \text { ) }\end{array}$ & 125480 & Fd & ?Manic-depressive illness (2) & \\
\hline $11 \mathrm{p} 15.5$ & $\begin{array}{l}\text { MTACR1, } \\
\text { WT2 }\end{array}$ & $\mathbf{P}$ & $\begin{array}{l}\text { Multiple tumor associated } \\
\text { chromosome region-1 }\end{array}$ & 194071 & $\mathrm{D}$ & Wilms tumor, type 2 (2) & \\
\hline $11 \mathrm{p} 15.5$ & $\begin{array}{l}\text { RMS, } \\
\text { RMSCR, } \\
\text { RMS1, } \\
\text { RMSE }\end{array}$ & $\mathbf{P}$ & Rhabdomyosarcoma, embryonal & 268210 & D & Rhabdomyosarcoma (2) & \\
\hline $11 \mathrm{p} 15.4$ & LDHA & $\mathrm{C}$ & Lactate dehydrogenase $\mathrm{A}$ & 150000 & S, D, REb, C, & $\begin{array}{l}\text { Exertional myoglobinuria due to } \\
\text { deficiency of LDH-A (1) }\end{array}$ & $7(\mathrm{Ldh}-1)$ \\
\hline $11 \mathrm{p} 15.4-15.1$ & $\begin{array}{l}\text { SMPD 1, } \\
\text { NPD }\end{array}$ & $\mathbf{P}$ & $\begin{array}{l}\text { Sphingomyelinase (Niemann-Pick } \\
\text { disease) }\end{array}$ & 257200 & $\mathrm{REa}, \mathrm{A}$ & Niemann-Pick disease (1) & \\
\hline $11 \mathrm{p} 15.3-\mathrm{p} 15.1$ & PTH & C & Parathyroid hormone & 168450 & $\begin{array}{l}\text { REa, REb, A, } \\
\text { Fd }\end{array}$ & Hypoparathyroidism, familial (1) & 7 (Pth) \\
\hline $11 \mathrm{p} 15$ & $\begin{array}{l}\text { FCP, HPFH, } \\
\text { HHPF }\end{array}$ & $\mathbf{L}$ & F-cell production & 142470 & $\mathbf{F}$ & $\begin{array}{l}\text { Heterocellular hereditary } \\
\text { persistence of fetal hemoglobin } \\
\text { (2) }\end{array}$ & \\
\hline $11 \mathrm{p} 15$ & $\begin{array}{l}\text { RBTN1, } \\
\text { RHOM1 }\end{array}$ & C & Rhombotin-1 & 186921 & $\mathrm{Ch}, \mathrm{D}$ & $\begin{array}{l}\text { Leukemia, T-cell acute } \\
\text { lymphoblastic (2) }\end{array}$ & \\
\hline $11 \mathrm{p} 14-\mathrm{p} 13$ & CD59 & $\mathbf{P}$ & Antigen CD59 (p18-20) & 107271 & REa & $\begin{array}{l}\text { Paroxysmal nocturnal } \\
\text { hemoglobinuria (1) }\end{array}$ & $15(\mathrm{Ly}-6)$ \\
\hline $11 \mathrm{p} 14-\mathrm{p} 13$ & $\begin{array}{l}\text { HVBS1, } \\
\text { HBVS1 }\end{array}$ & $\mathrm{C}$ & Hepatitis B virus integration site- 1 & 114550 & $\mathrm{REa}, \mathrm{A}, \mathrm{Ch}$ & Liver cell carcinoma (1) & \\
\hline $11 \mathrm{p} 13$ & CAT & C & Catalase & 115500 & $\mathrm{~S}, \mathrm{D}, \mathrm{Fd}$ & Acatalasemia (1) & 2(Cas-1) \\
\hline $11 \mathrm{p} 13$ & FSHB & $\mathrm{C}$ & $\begin{array}{l}\text { Follicle-stimulating hormone, beta } \\
\text { polypeptide }\end{array}$ & 136530 & $\mathrm{D}, \mathrm{REa}$ & ?Male infertility, familial (1) & $2($ Fshb) \\
\hline $11 \mathrm{p} 13$ & GUD & $\mathbf{P}$ & Genitourinary dysplasia & 137357 & $\mathrm{Ch}$ & Genitourinary dysplasia (2) & \\
\hline $11 \mathrm{p} 13$ & PAX6, AN2 & C & Paired box homeotic gene- 6 & 106210 & $\mathrm{Ch}, \mathrm{Fd}$ & Aniridia-2 (3) & 2 (Sey) \\
\hline $11 \mathrm{p} 13$ & TCL2 & $\mathbf{P}$ & T-cell leukemia/lymphoma-2 & 151390 & $\begin{array}{l}\mathrm{Ch}, \mathrm{RE}, \mathrm{A} \\
\mathrm{REa}\end{array}$ & Leukemia, acute $\mathrm{T}$-cell (2) & \\
\hline $11 \mathrm{p} 13$ & $\begin{array}{l}\text { WT1, } \\
\text { WAGR, } \\
\text { WTCR1 }\end{array}$ & $\mathrm{C}$ & $\begin{array}{l}\text { Wilms tumor } 1 \text { (Wilms tumor, } \\
\text { aniridia, gonadoblastoma, } \\
\text { retardation complex) }\end{array}$ & 194070 & $\mathrm{Ch}$ & $\begin{array}{l}\text { Wilms tumor (2); Aniridia of } \\
\text { WAGR syndrome (2); } \\
\text { Gonadoblastoma (2); Mental } \\
\text { retardation of WAGR (2) }\end{array}$ & $2(\mathrm{Wt}-1)$ \\
\hline $11 \mathrm{p} 12-\mathrm{p} 11$ & ACP2 & $\mathrm{C}$ & Acid phosphatase- 2 & 171650 & $\mathrm{~S}, \mathrm{REa}$ & $\begin{array}{l}\text { ?Lysosomal acid phosphatase } \\
\text { deficiency (1) }\end{array}$ & $2($ Acp-2) \\
\hline $11 \mathrm{p} 11-\mathrm{q} 12$ & F2 & $\mathrm{C}$ & Prothrombin (clotting factor II) & 176930 & REa, A & $\begin{array}{l}\text { Hypoprothrombinemia (1); } \\
\text { Dysprothrombinemia (1) }\end{array}$ & $2(\mathrm{Cf}-2)$ \\
\hline $11 \mathrm{q}$ & JBS & $\mathrm{L}$ & Jacobsen syndrome & 147791 & $\mathrm{Ch}$ & ?Jacobsen syndrome (2) & \\
\hline $11 \mathrm{q}$ & PC & $\mathbf{P}$ & Pyruvate carboxylase & 266150 & $\mathrm{REa}, \mathrm{H}$ & Pyruvate carboxylase deficiency (1) & 19(Pc) \\
\hline $11 \mathrm{q}$ & PORC & $\mathbf{P}$ & Porphyria, Chester type & 176010 & Fd & Porphyria, Chester type (2) & \\
\hline $11 \mathrm{q} 11-\mathrm{q} 13.1$ & $\begin{array}{l}\text { C1NH, C1I, } \\
\text { HANE }\end{array}$ & C & $\begin{array}{l}\text { Complement component-1 } \\
\text { inhibitor }\end{array}$ & 106100 & $\mathrm{REa}, \mathrm{A}$ & Angioedema, hereditary (1) & \\
\hline $11 q 12-q 13$ & APY, IGEL & $\mathrm{C}$ & $\begin{array}{l}\text { Atopy (allergic asthma and rhinitis; } \\
\text { immunoglobulin E level) }\end{array}$ & 147050 & Fd & Atopy (2) & \\
\hline $11 q 13$ & $\begin{array}{l}\text { CCND1, } \\
\text { PRAD1, } \\
\text { D11S287E }\end{array}$ & C & Cyclin D1 & 168461 & $\begin{array}{l}\text { REn, R, REa, } \\
\text { A }\end{array}$ & $\begin{array}{l}\text { Parathyroid adenomatosis } 1 \text { (2); } \\
\text { Centrocytic lymphoma (2) }\end{array}$ & \\
\hline $11 \mathrm{q} 13$ & MEN1 & $\mathrm{C}$ & $\begin{array}{l}\text { Multiple endocrine neoplasia, type } \\
\text { I }\end{array}$ & 131100 & Fd, D & Multiple endocrine neoplasia I (1) & \\
\hline $11 \mathrm{q} 13$ & PYGM, MGP & $\mathrm{C}$ & Phosphorylase, glycogen, muscle & 232600 & $\mathrm{REb}, \mathrm{Fd}$ & McArdle disease (1) & 19(Pygm) \\
\hline $11 \mathrm{q} 13$ & ST3, TSHL & C & $\begin{array}{l}\text { Suppression of tumorigenicity-3 } \\
\text { (tumor-suppressor gene, HELA } \\
\text { cell type) }\end{array}$ & 191181 & S, D & Cervical carcinoma (2) & \\
\hline $11 q 13$ & VMD2 & $\mathbf{P}$ & $\begin{array}{l}\text { Vitelliform macular dystrophy } \\
\text { (Best disease) }\end{array}$ & 153700 & Fd & $\begin{array}{l}\text { Macular dystrophy, vitelliform type } \\
\text { (2) }\end{array}$ & \\
\hline $11 q 13-q 23$ & EVR, FEVR & $\mathbf{P}$ & $\begin{array}{l}\text { Exudative vitreoretinopathy, } \\
\text { familial (Criswick-Schepens } \\
\text { syndrome) }\end{array}$ & 133780 & Fd & $\begin{array}{l}\text { Vitreoretinopathy, exudative, } \\
\text { familial (2) }\end{array}$ & \\
\hline $11 \mathrm{q} 13.3$ & BCL1 & C & B-cell CLL/lymphoma-1 & 151400 & $\mathrm{RE}, \mathrm{Ch}$ & Leukemia/lymphoma, B-cell, 1 (2) & \\
\hline $11 \mathrm{q} 14-\mathrm{q} 21$ & TYR, ATN & C & $\begin{array}{l}\text { Tyrosinase (albinism, tyrosinase } \\
\text { negative) }\end{array}$ & 203100 & $\mathrm{REa}, \mathrm{A}, \mathrm{H}, \mathrm{F}$ & Albinism (3) & $7(\mathrm{Tyr})$ \\
\hline $11 q 22-q 23$ & ATA, AT1 & C & $\begin{array}{l}\text { Ataxia-telangiectasia } \\
\text { (complementation groups A, C, } \\
\text { D) }\end{array}$ & 208900 & $\mathbf{F d}, \mathrm{C}, \mathrm{M}$ & Ataxia-telangiectasia (2) & \\
\hline $11 \mathrm{q} 22$-qter & ANC & $\mathbf{L}$ & Anal canal carcinoma & 105580 & $\mathrm{Ch}$ & ?Anal canal carcinoma (2) & \\
\hline $11 \mathrm{q} 22.3-\mathrm{q} 23.1$ & ACAT & $\mathbf{P}$ & $\begin{array}{l}\text { Acetyl-Coenzyme A } \\
\text { acetyltransferase (acetoacetyl } \\
\text { Coenzyme A thiolase) }\end{array}$ & 203750 & A & 3-ketothiolase deficiency (1) & \\
\hline
\end{tabular}


Table 1-contd

\begin{tabular}{|c|c|c|c|c|}
\hline Location & Symbol & Status & Title & MIM\# \\
\hline $11 \mathrm{q} 23$ & APOA1 & C & Apolipoprotein A-I & 107680 \\
\hline $11 q 23$ & MLL & $\mathbf{P}$ & $\begin{array}{l}\text { Myeloid/lymphoid, or } \\
\text { mixed-lineage leukemia }\end{array}$ & 159555 \\
\hline $11 \mathrm{q} 23$ & TSC2 & L & Tuberous sclerosis-2 & 191090 \\
\hline 11q23-qter & PGL, CBT1 & $\mathbf{P}$ & $\begin{array}{l}\text { Paraganglioma (carotid body } \\
\text { tumors) }\end{array}$ & 168000 \\
\hline $11 \mathrm{q} 24.1-\mathrm{q} 24.2$ & PBGD, UPS & C & $\begin{array}{l}\text { Porphobilinogen deaminase } \\
\text { (uroporphyrinogen I synthase) }\end{array}$ & 176000 \\
\hline Chr.11 & GLAU1 & $\mathbf{L}$ & Congenital glaucoma-1 & 231300 \\
\hline Chr.11 & GIF & $\mathbf{P}$ & Gastric intrinsic factor & 261000 \\
\hline 12pter-p12 & $\begin{array}{l}\text { F8VWF, } \\
\text { VWF }\end{array}$ & C & $\begin{array}{l}\text { Coagulation factor VIIII VWF (von } \\
\text { Willebrand factor) }\end{array}$ & 193400 \\
\hline 12 pter-q12 & BCT1 & C & Branched-chain aminotransferase-1 & 113520 \\
\hline $12 \mathrm{p} 13.3-\mathrm{p} 12.3$ & A2M & $\mathrm{C}$ & Alpha-2-macroglobulin & 103950 \\
\hline $12 \mathrm{p} 13$ & C1R & $\mathrm{C}$ & $\begin{array}{l}\text { Complement component-1, } \mathrm{r} \\
\text { subcomponent }\end{array}$ & 216950 \\
\hline $12 \mathrm{p} 13$ & C1S & C & $\begin{array}{l}\text { Complement component-1, s } \\
\text { subcomponent }\end{array}$ & 120580 \\
\hline $12 \mathrm{p} 13$ & MPE, EMP & $\mathbf{L}$ & $\begin{array}{l}\text { Eosinophils, malignant } \\
\text { proliferation of }\end{array}$ & 131440 \\
\hline $12 \mathrm{p} 13$ & TPI1,TPI & C & Triosephosphate isomerase & 190450 \\
\hline $12 \mathrm{p} 12.1$ & $\begin{array}{l}\text { KRAS2, } \\
\text { RASK2 }\end{array}$ & C & $\begin{array}{l}\text { Kirsten rat sarcoma-2 viral } \\
(v-\mathrm{Ki}-\mathrm{ras} 2) \text { oncogene homolog }\end{array}$ & 190070 \\
\hline $12 \mathrm{p} 12.1-\mathrm{p} 11.2$ & PTHLH & $\mathbf{P}$ & Parathyroid hormone-like hormone & 168470 \\
\hline $12 q 11-q 13$ & $\begin{array}{l}\text { KRT1, } \\
\text { KRTA }\end{array}$ & C & Keratin-1, alpha & 139350 \\
\hline
\end{tabular}

$\begin{array}{lllll}12 q 11-q 13 & \text { KRT5 } & \text { P } & \text { Keratin-5 } & 148040 \\ 12 q 12-q 14 & \text { VDR } & \text { P } & \begin{array}{c}\text { Vitamin D (1,25-dihydroxyvitamin } \\ \text { D3) receptor }\end{array} & 277440 \\ 12 q 13-q 14 & \text { BABL, LIPO } & \text { C } \begin{array}{c}\text { Lipoma (breakpoint in benign } \\ \text { lipoma); myxoid liposarcoma }\end{array} & 151900 \\ 12 q 13.11-q 13.2 & \text { COL2A1 } & \text { C } \begin{array}{c}\text { Collagen II, alpha-1 polypeptide } \\ 120140\end{array} & \end{array}$

A

REa, A

$\mathrm{Ch}$

REa, A

Method Disorder $\quad$ Mouse

REa, RE, Fd, ApoA-I and apoC-III deficiency, 9(Apoa-1) combined (1);

Hypertriglyceridemia, one form (1); Hypoalphalipoproteinemia (1); Amyloidosis, Iowa type, 107680.0010 (2)

Ch, RE Leukemia, myeloid/lymphoid or mixed-lineage (2)

$\mathrm{Ch}, \mathrm{Fd} \quad$ ?Tuberous sclerosis-2 (2)

Fd Paraganglioma (2)

S, D Porphyria, acute intermittent (1) 9(Ups)

Ch Glaucoma, congenital (2)

REa Anemia, pernicious, congenital, due

to deficiency of intrinsic factor (1)

A, REa, REb, von Willebrand disease (1) Fd $S$ REa, A Emphysema due to

Hyperleucinemia-isoleucinemia or

alpha-2-macroglobulin deficiency (1)

$\mathrm{REa}, \mathrm{Fd}, \mathrm{RE}, \mathrm{Clr} / \mathrm{C} 1$ s deficiency, combined (1) A

REa, Fd, RE, C1r/C1s deficiency, combined (1) A
$\mathrm{Ch}$
?Eosinophilic myeloproliferative disorder (2)

S, D, R, REa Hemolytic anemia due to triosephosphate isomerase

6(Tpi-1) deficiency (1)

REa, A, Fd Colorectal adenoma (1); Colorectal 6(Kras-2) cancer (1)

REa, A ?Humoral hypercalcemia of 6(Pthlh)

H, REa, A Epidermolysis bullosa simplex, 15(Krt-2) generalized, 131900 (1); Epidermolytic hyperkeratosis, 113800 (2); ?Epidermolysis bullosa simplex, localized, 131800 (1)

Epidermolysis bullosa simplex, Dowling-Meara type, 131760 (3); ?Epidermolysis bullosa, Weber Cockayne type, 131800 (2)

Rickets, vitamin D-resistant (1)

Lipoma (2); Myxoid liposarcoma (2); ?Multiple lipomatosis (2)

Stickler syndrome (3); Spondyloepiphyseal dysplasia congenita (3); ?Kniest dysplasia (1); Langer-Saldino achondrogenesis-

hypochondrogenesis (1); Osteoarthrosis, precocious (3) $\begin{array}{ll}\text { A, REa } & \text { Sanfilippo syndrome D (1) } \\ \text { Fd } & \text { Pseudo-vitamin D dependency }\end{array}$ rickets 1 (2)

$\mathrm{REa}, \mathrm{A}$ [Histidinemia] (1) 10(Hstd)

$\mathrm{REa}$ Acyl-CoA dehydrogenase, short 5(Bcd-1)

chain, deficiency of (1)

Fd, Ch ?Tuberous sclerosis-3 (2)

REa, A, Fd Phenylketonuria (3); 10(Pah) [Hyperphenylalaninemia, mild] (3)

$\mathrm{REa}, \mathrm{A}, \mathrm{H} \quad$ Alcohol intolerance, acute (1);

?Fetal alcohol syndrome (1)

REa ?Bloom syndrome, 210900 (2)

REa Mevalonicaciduria (1)

Ch ?Moebius syndrome (2)

F, Fd Wilson disease (2)

$\mathrm{Ch} \quad$ ?Letterer-Siwe disease (2)

$\mathrm{Ch}$ Osteosarcoma,

retinoblastoma-related $(2)$

Ch, F, Fd Retinoblastoma (2)

Ch Megacolon (2)

REa, D Propionicacidemia, type I or pccA type (1)

LD ?Dubin-Johnson syndrome (2)

D Factor VII deficiency (1)

D, A, REa Factor X deficiency (1)

?HHH syndrome (2)

L Dubin-Johnson syndrom

F7

$13 q 34$

HHHS

C Coagulation factor $\mathrm{X}$

227500

227600 hyperammonemiahomocitrullinemia syndrome

14(Rb-1) 
Table 1-contd

\begin{tabular}{|c|c|c|c|c|c|c|c|}
\hline Location & Symbol & Status & Title & MIM\# & Method & Disorder & Mouse \\
\hline Chr.13 & $\begin{array}{l}\text { BRCD1, } \\
\text { DBC, } \\
\text { BCDS1 }\end{array}$ & $\mathbf{P}$ & Breast cancer, ductal, suppressor-1 & 211410 & $\mathrm{D}$ & Breast cancer, ductal (2) & \\
\hline Chr.13 & $\begin{array}{l}\text { UVDR, } \\
\text { ERCM2 }\end{array}$ & $\mathbf{P}$ & $\begin{array}{l}\text { UV-damage, excision repair of (XP } \\
\text { complementation group I) }\end{array}$ & 192060 & $S$ & $\begin{array}{l}\text { ?Xeroderma pigmentosum, one } \\
\text { type (1) }\end{array}$ & \\
\hline $14 \mathrm{q}$ & $\begin{array}{l}\text { USH1A, } \\
\text { USH1 }\end{array}$ & $\mathbf{P}$ & $\begin{array}{l}\text { Usher syndrome-1A (autosomal } \\
\text { recessive, severe) }\end{array}$ & 276900 & $\mathrm{Fd}$ & Usher syndrome, type $1 \mathrm{~A}(2)$ & \\
\hline $14 \mathrm{q} 11.2$ & TCRA & C & $\begin{array}{l}\text { T-cell antigen receptor, alpha } \\
\text { polypeptide }\end{array}$ & 186880 & $\mathrm{H}, \mathrm{REa}, \mathrm{A}$ & Leukemia/lymphoma, T-cell (3) & 14 (Tcra) \\
\hline $14 \mathrm{q} 12$ & $\begin{array}{l}\text { MYH7, } \\
\text { MYHCB }\end{array}$ & $\mathbf{P}$ & $\begin{array}{l}\text { Myosin, heavy polypeptide- } 7 \text {, } \\
\text { cardiac muscle, beta }\end{array}$ & 160760 & $\begin{array}{l}\text { REa, RE, D, } \\
\text { A }\end{array}$ & $\begin{array}{l}\text { Hypertrophic cardiomyopathy, one } \\
\text { form, } 192600 \text { (3) }\end{array}$ & \\
\hline $14 \mathrm{q} 13.1$ & NP, NP1 & $\mathrm{C}$ & Nucleoside phosphorylase & 164050 & S, D & $\begin{array}{l}\text { Nucleoside phosphorylase } \\
\text { deficiency, immunodeficiency } \\
\text { due to (1) }\end{array}$ & $14(\mathrm{~Np}-1,2)$ \\
\hline $14 q 21-q 31$ & GALC & $\mathbf{P}$ & Galactocerebrosidase & 245200 & $\mathrm{REa}, \mathrm{A}, \mathrm{H}$ & Krabbe disease (1) & $12(\mathrm{tw})$ \\
\hline $14 \mathrm{q} 22$ & CSNU & $\mathrm{L}$ & Cystinuria & 220100 & $\mathrm{Ch}$ & ?Cystinuria (2) & \\
\hline $14 \mathrm{q} 22-\mathrm{q} 23.2$ & SPTB, SPH1 & $\mathrm{C}$ & Spectrin, beta, erythrocytic & 182870 & $\begin{array}{l}\text { REb, F, H, } \\
\text { REa, A, RE }\end{array}$ & $\begin{array}{l}\text { Elliptocytosis-3 (2); } \\
\text { Spherocytosis-1 (3) }\end{array}$ & $12($ Sptb-1) \\
\hline $14 q 23-q 24.2$ & HOS & I & Holt-Oram syndrome & 142900 & $\mathrm{Ch}$ & ?Holt-Oram syndrome (2) & \\
\hline $14 \mathrm{q} 31$ & TSHR & $\mathrm{C}$ & $\begin{array}{l}\text { Thyroid-stimulating hormone } \\
\text { receptor }\end{array}$ & 275200 & REa, Fd, A & $\begin{array}{l}\text { Hypothyroidism, nongoitrous, due } \\
\text { to TSH resistance (1) }\end{array}$ & \\
\hline $14 \mathrm{q} 31-\mathrm{q} 32.1$ & CBG & $\mathbf{P}$ & Corticosteroid binding globulin & 122500 & A & [Transcortin deficiency] (1) & \\
\hline $14 \mathrm{q} 32$ & CKBE & $\mathbf{P}$ & $\begin{array}{l}\text { Creatine kinase, brain type, ectopic } \\
\text { expression of }\end{array}$ & 123270 & $\mathrm{~F}$ & $\begin{array}{l}\text { [Creatine kinase, brain type, ectopic } \\
\text { expression of] (2) }\end{array}$ & \\
\hline $14 q 32$ & SIV & $\mathrm{L}$ & Situs inversus viscerum & 270100 & $\mathrm{H}$ & ?Situs inversus viscerum (2) & \\
\hline $14 q 32$ & VP, PPOX & $\mathbf{P}$ & $\begin{array}{l}\text { Porphyria variegata } \\
\text { (protoporphyrinogen oxidase) }\end{array}$ & 176200 & $\mathbf{F}$ & Porphyria variegata $(2)$ & \\
\hline $14 q 32.1$ & AACT & C & Alpha-1-antichymotrypsin & 107280 & $\begin{array}{l}\mathrm{REa}, \mathrm{A}, \mathrm{Fd}, \\
\text { REn }\end{array}$ & $\begin{array}{l}\text { Alpha-1-antichymotrypsin } \\
\text { deficiency (1) }\end{array}$ & \\
\hline $14 q 32.1$ & PI, AAT & C & $\begin{array}{l}\text { Protease inhibitor } \\
\quad \text { (alpha-1-antitrypsin) }\end{array}$ & 107400 & $\begin{array}{l}\mathrm{F}, \mathrm{S}, \mathrm{A}, \mathrm{D} \\
\mathrm{EM}, \mathrm{Fd}\end{array}$ & $\begin{array}{l}\text { Emphysema-cirrhosis (1); } \\
\text { Hemorrhagic diathesis due to } \\
\text { 'antithrombin' Pittsburgh (1); } \\
\text { Emphysema (1) }\end{array}$ & 12(Aat) \\
\hline $14 \mathrm{q} 32.1$ & TCL1 & C & T-cell lymphoma-1 & 186960 & $\mathrm{Ch}, \mathrm{RE}$ & Leukemia/lymphoma, T-cell (2) & \\
\hline $14 q 32.33$ & IGH & C & $\begin{array}{l}\text { IMMUNOGLOBULIN HEAVY } \\
\text { CHAIN GENE CLUSTER }\end{array}$ & & $\mathrm{REa}, \mathrm{A}$ & $\begin{array}{l}\text { ?Combined variable } \\
\text { hypogammaglobulinemia (1) }\end{array}$ & 12(Igh) \\
\hline $14 \mathrm{q} 32.33$ & IGHR & $\mathrm{L}$ & $\begin{array}{l}\text { Immunoglobulin heavy chain } \\
\text { regulator }\end{array}$ & 144120 & $\mathbf{F}$ & $\begin{array}{l}\text { ?Hyperimmunoglobulin } \mathrm{G} 1 \\
\text { syndrome }(2)\end{array}$ & \\
\hline Chr.14 & MPS3C & L & Sanfilippo disease, type IIIC & 252930 & $\mathrm{Ch}$ & ?Sanfilippo disease, type IIIC (2) & \\
\hline Chr.14 & PYGL, PPYL & $\mathbf{P}$ & Phosphorylase, glycogen, liver & 232700 & REb & $\begin{array}{l}\text { Hers disease, or glycogen storage } \\
\text { disease VI (1) }\end{array}$ & 12(Pygl) \\
\hline Chr.14 & $\mathrm{RMCH}$ & $\mathbf{P}$ & Rod monochromacy & 216900 & $\mathrm{Ch}$ & Rod monochromacy (2) & \\
\hline $15 q 11$ & DLX1 & $\mathrm{L}$ & Dyslexia-1 & 127700 & Fc, Fd & ?Dyslexia-1 (2) & \\
\hline $15 q 11$ & PWCR, PWS & $\mathrm{C}$ & Prader-Willi syndrome & 176270 & $\mathrm{Ch}, \mathrm{D}$ & Prader-Willi syndrome (2) & \\
\hline $15 q 11-q 13$ & $\begin{array}{l}\text { ANCR, } \\
\text { AGMS }\end{array}$ & C & Angelman syndrome & 234400 & Ch, D & Angelman syndrome (2) & \\
\hline $15 q 11-q 13$ & ITO & $\mathbf{L}$ & Hypomelanosis of Ito & 146150 & $\mathrm{Ch}$ & ?Hypomelanosis of Ito (2) & \\
\hline $15 q 14-q 15$ & IVD & $\mathbf{P}$ & Isovaleryl CoA dehydrogenase & 243500 & $\mathrm{REa}$ & Isovalericacidemia (1) & \\
\hline $15 q 15$ & EPB42 & C & $\begin{array}{l}\text { Erythrocyte surface protein band } \\
\quad 4.2\end{array}$ & 177070 & A & $\begin{array}{l}\text { Spherocytosis, hereditary, Japanese } \\
\text { type (1) }\end{array}$ & \\
\hline $15 q 15-q 22$ & LGMD2 & $\mathrm{C}$ & $\begin{array}{l}\text { Limb-girdle muscular dystrophy, } \\
\text { autosomal recessive }\end{array}$ & 253600 & Fd & $\begin{array}{l}\text { Muscular dystrophy, limb-girdle, } \\
\text { autosomal recessive }(2)\end{array}$ & \\
\hline $15 q 21-q 22$ & B2M & $\mathrm{C}$ & Beta-2-microglobulin & 109700 & $\mathrm{~S}, \mathrm{D}, \mathrm{H}$ & $\begin{array}{l}\text { Hemodialysis-related amyloidosis } \\
\text { (1) }\end{array}$ & $2(\mathrm{~B} 2 \mathrm{~m})$ \\
\hline $15 q 21-q 23$ & $\begin{array}{l}\text { LIPC, LIPH, } \\
\text { HL, HTGL }\end{array}$ & $\mathrm{C}$ & Hepatic triglyceride lipase & 151670 & $\mathrm{REa}, \mathrm{A}$ & ?Hepatic lipase deficiency (1) & $9(\mathrm{Hl})$ \\
\hline $15 \mathrm{q} 21.1$ & CYP19, ARO & C & $\begin{array}{l}\text { Cytochrome P450, subfamily XIX } \\
\text { (aromatization of androgens) }\end{array}$ & 107910 & $\mathrm{REa}, \mathrm{A}, \mathrm{H}$ & $\begin{array}{l}\text { ?Gynecomastia, familial, due to } \\
\text { increased aromatase activity (1) }\end{array}$ & $9($ Cyp19) \\
\hline $15 q 21.1$ & $\begin{array}{l}\text { FBN1, FBN, } \\
\text { MFS1 }\end{array}$ & C & Fibrillin-1 & 134797 & $\mathrm{~A}, \mathrm{Fd}$ & Marfan syndrome, 154700 & \\
\hline $15 q 22$ & PML, MYL & $\mathbf{P}$ & $\begin{array}{l}\text { Acute promyelocytic leukemia, } \\
\text { inducer of }\end{array}$ & 102578 & $\mathrm{Ch}, \mathrm{RE}$ & Leukemia, acute promyelocytic (2) & \\
\hline $15 q 23-q 24$ & HEXA, TSD & C & $\begin{array}{l}\text { Hexosaminidase A (alpha } \\
\text { polypeptide) }\end{array}$ & 272800 & $\mathrm{~S}, \mathrm{D}, \mathrm{A}$ & $\begin{array}{l}\text { Tay-Sachs disease (1); } \\
\text { GM2-gangliosidosis, juvenile, } \\
\text { adult (1); [Hex A } \\
\text { pseudodeficiency] (1) }\end{array}$ & 9(Hexa) \\
\hline $15 q 23-q 25$ & ETFA, GA2 & $\mathbf{P}$ & $\begin{array}{l}\text { Electron transfer flavoprotein, } \\
\text { alpha polypeptide }\end{array}$ & 231680 & $\mathrm{REa}, \mathrm{A}$ & Glutaricaciduria, type II (1) & \\
\hline $15 q 23-q 25$ & FAH & C & Fumarylacetoacetase & 276700 & A, REa & Tyrosinemia, type I (1) & \\
\hline $15 q 24-q 25$ & CTSH & $\mathbf{P}$ & Cathepsin $\mathrm{H}$ & 116820 & $\mathrm{REa}, \mathrm{A}$ & $\begin{array}{l}\text { ?Batten disease, one form, } 204200 \\
\text { (1) }\end{array}$ & \\
\hline Chr.15 & $\mathrm{XPF}$ & $\mathbf{P}$ & Xeroderma pigmentosum, group F & 278760 & $\mathbf{M}$ & $\begin{array}{l}\text { Xeroderma pigmentosum, type } \mathrm{F} \\
\text { (2) }\end{array}$ & \\
\hline 16pter-p13.3 & HBA1 & C & Hemoglobin alpha-1 & 141800 & HS & $\begin{array}{l}\text { Thalassemias, alpha- (1); } \\
\text { Methemoglobinemias, alpha- (1); } \\
\text { Erythremias, alpha- (1); Heinz } \\
\text { body anemias, alpha- (1) }\end{array}$ & 11(Hba) \\
\hline 16pter-p13.3 & HBHR & $\mathbf{P}$ & $\mathrm{Hb} \mathrm{H}$ mental retardation syndrome & 141750 & $\mathrm{~F}$ & $\begin{array}{l}\text { Hb H mental retardation syndrome } \\
\text { (2) }\end{array}$ & \\
\hline $16 \mathrm{p} 13.31-\mathrm{p} 13.12$ & $\begin{array}{l}\text { PKD1, } \\
\text { APKD }\end{array}$ & C & Adult polycystic kidney disease & 173900 & $\mathrm{~F}, \mathrm{Fd}$ & Polycystic kidney disease (2) & ?17(Pkd-1) \\
\hline $16 \mathrm{p} 13.3$ & RSTS, RTS & $\mathrm{C}$ & Rubinstein-Taybi syndrome & 180849 & $\mathrm{Ch}$ & Rubinstein-Taybi syndrome (2) & \\
\hline $16 \mathrm{p} 13$ & $\begin{array}{l}\text { HAGH, } \\
\text { GLO2 }\end{array}$ & $\mathrm{C}$ & $\begin{array}{l}\text { Hydroxyacyl glutathione hydrolase; } \\
\text { glyoxalase II }\end{array}$ & 138760 & $\mathbf{S}$ & [Glyoxalase II deficiency] (1) & \\
\hline $16 \mathrm{p} 13$ & MEF, FMF & $\mathbf{P}$ & Familial Mediterranean fever & 249100 & $\mathrm{Fd}$ & Familial Mediterranean fever (2) & \\
\hline $16 \mathrm{p} 13$ & TSC4 & $\mathbf{P}$ & Tuberous sclerosis-4 & 191092 & Fd & Tuberous sclerosis-4 (2) & \\
\hline $16 \mathrm{p} 12$ & CLN3, BTS & $\mathrm{C}$ & Ceroid-lipofuscinosis, neuronal-3, & 204200 & $\mathrm{~F}, \mathrm{Fd}$ & Batten disease (2) & \\
\hline
\end{tabular}


Table 1-contd

\begin{tabular}{|c|c|c|c|c|c|c|c|}
\hline Location & Symbol & Status & Title & MIM\# & Method & Disorder & Mouse \\
\hline $16 q 12-q 13.1$ & PHKB & C & $\begin{array}{l}\text { Phosphorylase kinase, beta } \\
\text { polypeptide }\end{array}$ & 172490 & REa, A & $\begin{array}{l}\text { ?Phosphorylase kinase deficiency of } \\
\text { liver and muscle, } 261750 \text { (2) }\end{array}$ & \\
\hline $16 q 13-q 22.1$ & CES1, SES1 & $\mathbf{P}$ & Carboxyesterase-1 & 114835 & REa & $\begin{array}{l}\text { ?Monocyte carboxyesterase } \\
\text { deficiency (1) }\end{array}$ & $8($ Ces-1) \\
\hline $16 \mathrm{q} 21$ & CETP & $\mathbf{P}$ & $\begin{array}{l}\text { Cholesteryl ester transfer protein, } \\
\text { plasma }\end{array}$ & 118470 & REa, A & [CETP deficiency] (1) & \\
\hline $16 q 22-q 24$ & $\begin{array}{l}\text { ALDOA, } \\
\text { ALDA }\end{array}$ & C & $\begin{array}{l}\text { Aldolase A, } \\
\text { fructose-bisphosphatase }\end{array}$ & 103850 & REa, REb, A & ?Aldolase A deficiency (1) & \\
\hline $16 \mathrm{q} 22.1$ & $\begin{array}{l}\text { CTM, CPM, } \\
\text { CAM }\end{array}$ & C & Cataract, Marner type & 116800 & $\mathbf{F}$ & Cataract, Marner type (2) & \\
\hline $16 \mathrm{q} 22.1$ & LCAT & C & Lecithin-cholesterol acyltransferase & 245900 & $\begin{array}{l}\text { F, LD, A, } \\
\text { REa }\end{array}$ & $\begin{array}{l}\text { Norum disease (3); Fish-eye } \\
\text { disease (3) }\end{array}$ & 8 (Lcat) \\
\hline $16 \mathrm{q} 22.1-\mathrm{q} 22.3$ & TAT & C & Tyrosine aminotransferase, cytosolic & 276600 & REa, A, H, D & Tyrosinemia, type II (1) & 8 (Tat) \\
\hline $16 \mathrm{q} 24$ & APRT & C & Adenine phosphoribosyltransferase & 102600 & S, D & Urolithiasis, 2,8-dihydroxyadenine (1) & 8(Aprt) \\
\hline $16 \mathrm{q} 24$ & CYBA & C & $\begin{array}{l}\text { Cytochrome b-245, alpha } \\
\text { polypeptide }\end{array}$ & 233690 & $\mathrm{REa}, \mathrm{A}$ & $\begin{array}{l}\text { Chronic granulomatous disease, } \\
\text { autosomal, due to deficiency of } \\
\text { CYBA (3) }\end{array}$ & \\
\hline Chr.16 & $\begin{array}{r}\text { ATP2A1, } \\
\text { ATP2A }\end{array}$ & $\mathbf{P}$ & $\begin{array}{l}\text { ATPase, } \mathrm{Ca}++ \text { transporting, } \\
\text { fast-twitch, } 1\end{array}$ & 108730 & REa & Brody myopathy (1) & \\
\hline Chr.16 & CTH & $\mathbf{P}$ & Cystathionase & 219500 & $\mathbf{S}$ & [Cystathioninuria] (1) & \\
\hline 17 pter-p12 & GP1BA & $\mathbf{P}$ & $\begin{array}{l}\text { Glycoprotein Ib, platelet, alpha } \\
\text { polypeptide }\end{array}$ & 231200 & A & Bernard-Soulier syndrome (1) & \\
\hline 17 pter-p12 & PLI & $\mathbf{P}$ & Alpha-2-plasmin inhibitor & 262850 & Psh & Plasmin inhibitor deficiency (1) & \\
\hline $17 \mathrm{p} 13.3$ & BCPR & $\mathbf{L}$ & $\begin{array}{l}\text { Breast cancer-related regulator of } \\
\text { TP53 }\end{array}$ & 113721 & $\mathbf{D}$ & ?Breast cancer $(1)$ & \\
\hline $17 \mathrm{p} 13.3$ & $\begin{array}{l}\text { MDCR, } \\
\text { MDLS, } \\
\text { MDS }\end{array}$ & C & $\begin{array}{l}\text { Miller-Dieker lissencephaly } \\
\text { syndrome }\end{array}$ & 247200 & $\mathrm{Ch}, \mathrm{D}$ & $\begin{array}{l}\text { Miller-Dieker lissencephaly } \\
\text { syndrome (2) }\end{array}$ & $11(\mathrm{Mds})$ \\
\hline $17 \mathrm{p} 13.1$ & $\begin{array}{l}\text { TP53, } \\
\text { CRCR2, } \\
\text { CRC17 }\end{array}$ & C & Tumor protein p53 & 191170 & REa, A, D & $\begin{array}{l}\text { Colorectal cancer, } 114500(3) ; \\
\text { Li-Fraumeni syndrome (1) }\end{array}$ & $11(\operatorname{Trp} 53)$ \\
\hline $17 \mathrm{p} 11.2$ & CMT1A & C & $\begin{array}{l}\text { Charcot-Marie-Tooth disease, slow } \\
\text { nerve conduction type Ia }\end{array}$ & 118220 & Fd, D, A & $\begin{array}{l}\text { Charcot-Marie-Tooth neuropathy, } \\
\text { slow nerve conduction type Ia (2) }\end{array}$ & $11(\mathrm{Tr})$ \\
\hline $17 \mathrm{p} 11.2$ & SMCR & $\mathbf{C}$ & $\begin{array}{l}\text { Smith-Magenis syndrome } \\
\text { chromosome region }\end{array}$ & 182290 & $\mathrm{Ch}$ & Smith-Magenis syndrome (2) & \\
\hline $17 q 11-q 12$ & $\begin{array}{l}\text { EDH17B1, } \\
\text { EDHB17A }\end{array}$ & C & Estradiol 17-beta-dehydrogenase-1 & 264300 & A, REa & $\begin{array}{l}\text { Pseudohermaphroditism, male, } \\
\text { with gynecomastia (1); Polycystic } \\
\text { ovarian disease (1) }\end{array}$ & \\
\hline $17 q 11.2$ & NF1, VRNF & C & $\begin{array}{l}\text { Neurofibromatosis, von } \\
\text { Recklinghausen type }\end{array}$ & 162200 & $\begin{array}{l}\text { Fd, EM, Ch, } \\
\quad \text { F }\end{array}$ & $\begin{array}{l}\text { Neurofibromatosis, von } \\
\text { Recklinghausen (2) }\end{array}$ & \\
\hline $17 \mathrm{q} 11.2$ & WSS & $\mathbf{P}$ & Watson syndrome & 193520 & Fd & Watson syndrome (2) & \\
\hline $17 q 12-q 21$ & KRT14, K14 & $\mathbf{P}$ & Keratin-14 & 148066 & REa & $\begin{array}{l}\text { Epidermolysis bullosa simplex, } \\
131900 \text { (3); Epidermolysis } \\
\text { bullosa simplex, Dowling-Meara } \\
\text { type, } 131760 \text { (3) }\end{array}$ & \\
\hline $17 \mathrm{q} 21$ & ACAC, ACC & $\mathbf{P}$ & Acetyl-CoA carboxylase & 200350 & A & Acetyl-CoA carboxylase deficiency (1) & \\
\hline $17 q 21$ & BRCA1 & $\mathbf{P}$ & Breast cancer-1, early onset & 113705 & Fd & Breast cancer-1, early onset (2) & \\
\hline $17 q 21-q 22$ & $\begin{array}{l}\text { EPB3, } \\
\text { EMPB3 }\end{array}$ & C & $\begin{array}{l}\text { Erythroid membrane protein band } \\
3\end{array}$ & 109270 & REa, RE, Fd & $\begin{array}{l}\text { [Acanthocytosis, one form] (1); } \\
\text { [Elliptocytosis, } \\
\text { Malaysian-Melanesian type] (1) }\end{array}$ & \\
\hline $17 q 21-q 22$ & GALK & C & Galactokinase & 230200 & S, Ch, R, C & Galactokinase deficiency (1) & 11 (Glk) \\
\hline $17 q 21-q 22$ & KRT10 & C & Keratin-10 & 148080 & REa, A, REn & $\begin{array}{l}\text { Epidermolytic hyperkeratosis, } 113800 \\
\text { (1) }\end{array}$ & \\
\hline $17 q 21.1$ & RARA & C & $\begin{array}{l}\text { Retinoic acid receptor, alpha } \\
\text { polypeptide }\end{array}$ & 180240 & $\mathrm{~A}, \mathrm{Ch}$ & Leukemia, acute promyelocytic (1) & 11(Rara) \\
\hline $17 q 21.3-q 22$ & MPO & C & Myeloperoxidase & 254600 & $\begin{array}{l}\mathrm{REa}, \mathrm{A}, \mathrm{F} \\
\mathrm{Ch}, \mathrm{C}\end{array}$ & Myeloperoxidase deficiency (1) & $11(\mathrm{Mpo})$ \\
\hline $17 \mathrm{q} 21.31-\mathrm{q} 22.05$ & COL1A1 & C & Collagen I, alpha-1 polypeptide & 120150 & C, M, A, REa & $\begin{array}{l}\text { Osteogenesis imperfecta, } 2 \text { or more } \\
\text { clinical forms (3); Ehlers-Danlos } \\
\text { syndrome, type VIIAl (3) }\end{array}$ & 11(Cola-1) \\
\hline $17 q 21.32$ & $\begin{array}{l}\text { ITGA2B, } \\
\text { GP2B, } \\
\text { CD41B }\end{array}$ & C & $\begin{array}{l}\text { Integrin, alpha IIB (platelet } \\
\text { glycoprotein IIb of IIb/IIIa } \\
\text { complex, antigen CD41B) }\end{array}$ & 273800 & $\begin{array}{l}\text { A, REb, REa, } \\
\text { RE, F, LD }\end{array}$ & $\begin{array}{l}\text { Glanzmann thrombasthenia, type } \mathbf{A} \\
\text { (1) }\end{array}$ & \\
\hline $17 q 21.32$ & $\begin{array}{l}\text { ITGB3, } \\
\text { GP3A }\end{array}$ & C & $\begin{array}{l}\text { Integrin, beta-3 (platelet } \\
\text { glycoprotein IIIa; antigen CD61) }\end{array}$ & 173470 & $\begin{array}{l}\text { REa, REb, A, } \\
\text { RE, F, LD }\end{array}$ & $\begin{array}{l}\text { Glanzmann thrombasthenia, type B } \\
\text { (1) }\end{array}$ & \\
\hline $17 q 22-q 24$ & $\begin{array}{l}\text { CSH1, CSA, } \\
\text { PL }\end{array}$ & C & Chorionic somatomammotropin A & 150200 & $\mathrm{REa}, \mathrm{A}$ & [Placental lactogen deficiency] (1) & 13(Pl-1) \\
\hline $17 q 22-q 24$ & GH1, GHN & C & Growth hormone, normal & 139250 & REa, A, Fd & $\begin{array}{l}\text { Isolated growth hormone } \\
\text { deficiency, Illig type with absent } \\
\text { GH and Kowarski type with } \\
\text { bioinactive GH (3) }\end{array}$ & $11(\mathrm{Gh})$ \\
\hline $17 q 23$ & GAA & C & Acid alpha-glucosidase & 232300 & $S, A, D, C$ & $\begin{array}{l}\text { Pompe disease (1); Acid-maltase } \\
\text { deficiency, adult (1) }\end{array}$ & \\
\hline 17q23-qter & APOH & C & $\begin{array}{l}\text { Apolipoprotein } \mathrm{H} \\
\text { (beta-2-glycoprotein I) }\end{array}$ & 138700 & $\mathrm{Fd}, \mathrm{REa}$ & [Apolipoprotein H deficiency] (1) & 11(Apoh) \\
\hline $17 q 23.1-q 25.3$ & $\begin{array}{l}\text { SCN4A, } \\
\text { HYPP, } \\
\text { NAC1A }\end{array}$ & C & $\begin{array}{l}\text { Sodium channel, voltage-gated, } \\
\text { type } 4 \text {, alpha polypeptide }\end{array}$ & 170500 & $\mathbf{R E a}, \mathbf{F d}$ & $\begin{array}{l}\text { Hyperkalemic periodic paralysis } \\
\text { (3); Paramyotonia congenita (3); } \\
\text { Myotonia congenita, atypical } \\
\text { acetazolamide-responsive (3) }\end{array}$ & \\
\hline $18 \mathrm{p} 11.32$ & MCL & $\mathrm{L}$ & $\begin{array}{l}\text { Multiple hereditary cutaneous } \\
\text { leiomyomata }\end{array}$ & 150800 & $\mathrm{Ch}$ & $\begin{array}{l}\text { ?Leiomyomata, multiple hereditary } \\
\text { cutaneous }(2)\end{array}$ & \\
\hline $18 q 11-q 12$ & LCFS2 & $\mathbf{L}$ & Lynch cancer family syndrome II & 114400 & $\mathbf{F}$ & $\begin{array}{l}\text { ?Lynch cancer family syndrome II } \\
\text { (2) }\end{array}$ & \\
\hline $18 \mathrm{q} 11.2-\mathrm{q} 12.1$ & $\begin{array}{l}\text { TTR, PALB, } \\
\text { TBPA }\end{array}$ & C & $\begin{array}{l}\text { Thyroxine-binding prealbumin } \\
\text { (transthyretin) }\end{array}$ & 176300 & $\mathrm{REa}, \mathrm{A}$ & $\begin{array}{l}\text { Amyloid neuropathy, familial, } \\
\text { several allelic types (1); } \\
\text { [Dystransthyretinemic } \\
\text { hyperthyroxinemia](1); } \\
\text { Amyloidosis, senile systemic (1) }\end{array}$ & 18(Palb) \\
\hline
\end{tabular}


Table 1-contd

\begin{tabular}{|c|c|c|c|c|c|c|c|}
\hline Location & Symbol & Status & Title & MIM\# & Method & Disorder & Mouse \\
\hline $18 \mathrm{q} 21-\mathrm{q} 22.2$ & $\begin{array}{l}\text { CORD, } \\
\text { RCRD1 }\end{array}$ & $\mathbf{L}$ & Retinal cone-rod dystrophy-1 & 120970 & $\mathrm{Ch}$ & ?Retinal cone-rod dystrophy (2) & \\
\hline $18 \mathrm{q} 21.3$ & BCL2 & C & B-cell CLL/lymphoma-2 & 151430 & $\mathrm{Ch}, \mathrm{RE}, \mathrm{REn}$ & Leukemia/lymphoma, B-cell, 2 (2) & $1(\mathrm{Bcl}-2)$ \\
\hline $18 \mathrm{q} 21.3$ & FECH, FCE & C & Ferrochelatase & 177000 & $\mathrm{~A}, \mathrm{REb}$ & Protoporphyria, erythropoietic (1) & \\
\hline $18 \mathrm{q} 22.1$ & GTS & $\mathbf{L}$ & Gilles de la Tourette syndrome & 137580 & $\mathrm{Ch}$ & ?Tourette syndrome (2) & \\
\hline $18 \mathrm{q} 23.3$ & DCC, CRC18 & $\mathrm{C}$ & $\begin{array}{l}\text { Colorectal cancer-related } \\
\text { sequence-18 }\end{array}$ & 120470 & $\mathrm{D}, \mathrm{RE}$ & Colorectal cancer (1) & $18(\mathrm{Dcc})$ \\
\hline Chr.18 & CYB5 & $\mathbf{P}$ & Cytochrome b5 & 250790 & Psh & $\begin{array}{l}\text { Methemoglobinemia due to } \\
\text { cytochrome b5 deficiency (1) }\end{array}$ & \\
\hline $19 \mathrm{p} 13.3-\mathrm{p} 13.2$ & AMH, MIF & $P$ & Anti-Mullerian hormone & 261550 & REa, A & $\begin{array}{l}\text { Persistent Mullerian duct } \\
\text { syndrome (1) }\end{array}$ & $10(\mathrm{Amh})$ \\
\hline 19p13.3-p13.2 & ATHS, ALP & $\mathbf{P}$ & $\begin{array}{l}\text { Atherogenic lipoprotein phenotype } \\
\text { (atherosclerosis susceptibility) }\end{array}$ & 108725 & $\mathrm{Fd}$ & Atherosclerosis, susceptibility to (2) & \\
\hline 19p13.3-p13.2 & C3 & C & Complement component-3 & 120700 & F, S, A, REa & C3 deficiency (1) & $17(\mathrm{C} 3)$ \\
\hline $19 \mathrm{p} 13.3-\mathrm{p} 13.2$ & INSR & C & Insulin receptor & 147670 & $\mathrm{REa}, \mathrm{A}, \mathrm{REb}$ & $\begin{array}{l}\text { Leprechaunism (1); Diabetes } \\
\text { mellitus, insulin-resistant, with } \\
\text { acanthosis nigricans (1); ?Rabson- } \\
\text { Mendenhall syndrome (1) }\end{array}$ & $8($ Insr $)$ \\
\hline $19 \mathrm{p} 13.3-\mathrm{p} 13.2$ & TCF3, E2A & $\mathbf{P}$ & $\begin{array}{l}\text { Transcription factor-3 (E2A } \\
\text { immunoglobulin enhancer } \\
\text { binding factors E12/E47) }\end{array}$ & 147141 & REa, A & Leukemia, acute lymphoblastic (1) & \\
\hline 19p13.2-p13.1 & LDLR,FHC & $\mathrm{C}$ & $\begin{array}{l}\text { Familial hypercholesterolemia } \\
\text { (LDL receptor) }\end{array}$ & 143890 & F, REa, A & Hypercholesterolemia, familial (3) & 9(Ldlr) \\
\hline $19 \mathrm{p} 13.2-\mathrm{q} 12$ & MANB & C & Lysosomal alpha-D-mannosidase-B & 248500 & $\mathbf{S}$ & Mannosidosis (1) & \\
\hline $19 \mathrm{p} 13.1$ & RFX1 & C & $\begin{array}{l}\text { Regulatory factor (trans-acting) } 1 \\
\text { (influences HLA class II } \\
\text { expression) }\end{array}$ & 209920 & A & $\begin{array}{l}\text { Severe combined } \\
\text { immunodeficiency, HLA class } \\
\text { II-negative type (1) }\end{array}$ & \\
\hline $19 \mathrm{p} 13$ & LYL1 & $\mathbf{P}$ & Leukemia, lymphoid, 1 & 151440 & $\mathrm{Ch}$ & $\begin{array}{l}\text { Leukemia, T-cell acute } \\
\text { lymphoblastoid }(2)\end{array}$ & $8($ Lyl-1) \\
\hline 19 cen-q12 & GPI & C & $\begin{array}{l}\text { Glucose phosphate isomerase; } \\
\text { neuroleukin }\end{array}$ & 172400 & S, D & $\begin{array}{l}\text { Hemolytic anemia due to } \\
\text { glucosephosphate isomerase } \\
\text { deficiency (1); Hydrops fetalis, } \\
\text { one form (1) }\end{array}$ & $7(\mathrm{Gpi}-1)$ \\
\hline 19 cen-q13.11 & PEPD & C & Peptidase D (prolidase) & 170100 & $\mathrm{~S}, \mathrm{~F}, \mathrm{H}, \mathrm{Fd}$ & Prolidase deficiency (1) & 7(Pep-4) \\
\hline $19 q 12-q 13.2$ & $\mathrm{CCO}, \mathrm{CCD}$ & C & Central core disease of muscle & 117000 & Fd & Central core disease of muscle (2) & \\
\hline $19 \mathrm{q} 12-\mathrm{q} 13.2$ & PVS & C & Polio virus sensitivity & 173850 & S & $\{$ Polio, susceptibility to $\}$ (2) & 9(Pvs) \\
\hline $19 q 13$ & BCL3 & C & B-cell CLL/lymphoma-3 & 109560 & $\mathrm{Ch}, \mathrm{S}, \mathrm{H}$ & Leukemia/lymphoma, B-cell, 3 (2) & $7(\mathrm{Bcl}-3)$ \\
\hline $19 \mathrm{q} 13.1$ & APOE & C & Apolipoprotein E & 107741 & F, REa, LD, & Hyperlipoproteinemia, type III (1) & 7(Apoe) \\
\hline $19 \mathrm{q} 13.1$ & APOC2 & $\mathrm{C}$ & Apolipoprotein C-II & 207750 & $\begin{array}{l}\text { REa, F, LD, } \\
\quad \text { A, Fd }\end{array}$ & Hyperlipoproteinemia, type Ib (1) & \\
\hline $19 \mathrm{q} 13.1$ & $\begin{array}{l}\text { RYR1, } \\
\text { RYDR, } \\
\text { MHS }\end{array}$ & C & $\begin{array}{l}\text { Ryanodine receptor (sarcoplasmic } \\
\text { reticulum calcium release } \\
\text { channel) }\end{array}$ & 180901 & $\mathrm{~A}, \mathrm{Fd}, \mathrm{H}$ & $\begin{array}{l}\text { Malignant hyperthermia, } 145600 \\
\text { (3) }\end{array}$ & 7 (Ryr) \\
\hline $19 q 13.1-q 13.2$ & $\begin{array}{l}\text { BCKDHA, } \\
\text { MSUD1 }\end{array}$ & $\mathrm{C}$ & $\begin{array}{l}\text { Branched-chain keto acid } \\
\text { dehydrogenase E1, alpha } \\
\text { polypeptide }\end{array}$ & 248600 & $\mathrm{REa}, \mathrm{REb}, \mathrm{A}$ & $\begin{array}{l}\text { Maple syrup urine disease, type } 1 \\
\text { (3) }\end{array}$ & \\
\hline $19 q 13.2-q 13.3$ & DM & $\mathrm{C}$ & Myotonic dystrophy & 160900 & F, Fd & Myotonic dystrophy (2) & \\
\hline $19 q 13.2-q 13.3$ & ERCC2, EM9 & $\mathrm{C}$ & $\begin{array}{l}\text { Excision repair cross complementing } \\
\text { rodent repair deficiency, } \\
\text { complementation group- } 2\end{array}$ & 126340 & S, RE & $\begin{array}{l}\text { Xeroderma pigmentosum, group } \\
\mathrm{D}, 278730 \text { (1) }\end{array}$ & $7($ Ercc-2) \\
\hline $19 q 13.3$ & LIG1 & C & Ligase I, DNA, ATP-dependent & 126391 & REa, A & DNA ligase I deficiency (1) & \\
\hline $19 \mathrm{q} 13.32$ & LHB & C & $\begin{array}{l}\text { Luteinizing hormone, beta } \\
\text { polypeptide }\end{array}$ & 152780 & RE & $\begin{array}{l}\text { Hypogonadism, hypergonadotropic } \\
\text { (1); ?Male pseudohermaphroditism } \\
\text { due to defective LH (1) }\end{array}$ & $7(\mathrm{Lhb})$ \\
\hline Chr.19 & ВCT2 & $\mathbf{P}$ & Branched-chain aminotransferase- 2 & 113530 & $\mathrm{~S}$ & $\begin{array}{l}\text { ?Hypervalinemia or } \\
\text { hyperleucine-isoleucinemia (1) }\end{array}$ & \\
\hline Chr.19 & ETFB & $\mathbf{P}$ & $\begin{array}{l}\text { Electron transfer flavoprotein, beta } \\
\text { polypeptide }\end{array}$ & 130410 & REa & Glutaricaciduria, type IIc (1) & \\
\hline 20pter-p12.21 & ARVP, VP & $\mathbf{P}$ & $\begin{array}{l}\text { Arginine vasopressin-neurophysin } \\
\text { II }\end{array}$ & 192340 & $\mathrm{REa}, \mathrm{RE}, \mathrm{Fd}$ & $\begin{array}{l}\text { Diabetes insipidus, } \\
\text { neurohypophyseal, } 125700 \text { (1) }\end{array}$ & \\
\hline 20pter-p12 & PRNP, PRIP & $\mathrm{C}$ & Prion protein $(\mathrm{p} 27-30)$ & 176640 & $\mathrm{REa}, \mathrm{REb}, \mathrm{A}$ & $\begin{array}{l}\text { Creutzfeldt-Jakob disease, } 123400 \\
\text { (3); Gerstmann-Straussler } \\
\text { disease, } 137440 \text { (3); Insomnia, } \\
\text { fatal familial (3) }\end{array}$ & $2(\operatorname{Prn}-\mathrm{p})$ \\
\hline $20 \mathrm{p} 13$ & HOS & I & Holt-Oram syndrome & 142900 & $\mathrm{Ch}$ & ?Holt-Oram syndrome (2) & \\
\hline 20 p11.23-qter & GHRF & C & $\begin{array}{l}\text { Growth hormone releasing factor; } \\
\text { somatocrinin }\end{array}$ & 139190 & $\begin{array}{l}\mathrm{REa}, \mathrm{REb} \\
\mathrm{Ch}\end{array}$ & $\begin{array}{l}\text { ?Isolated growth hormone } \\
\text { deficiency due to defect in } \\
\text { GHRF (1) }\end{array}$ & \\
\hline $20 \mathrm{p} 11.2$ & AGS, AHD & $\mathrm{C}$ & $\begin{array}{l}\text { Alagille syndrome (arteriohepatic } \\
\text { dysplasia) }\end{array}$ & 118450 & $\mathrm{Ch}, \mathrm{D}$ & Alagille syndrome (2) & \\
\hline 20p11 & CST3 & C & Cystatin C & 105150 & $\mathrm{REa}, \mathrm{A}$ & Cerebral amyloid angiopathy (1) & \\
\hline $20 p$ & ITPA & C & Inosine triphosphatase-A & 147520 & S & $\begin{array}{l}\text { [Inosine triphosphatase deficiency] } \\
\text { (1) }\end{array}$ & 2 (Itp) \\
\hline $20 \mathrm{q} 13$ & MODY1 & C & $\begin{array}{l}\text { Maturity-onset diabetes of the } \\
\text { young, type I }\end{array}$ & 125850 & $\mathrm{Fd}$ & MODY, type I (2) & \\
\hline $20 \mathrm{q} 13.1$ & $\begin{array}{l}\text { PPGB, GSL, } \\
\text { NGBE, } \\
\text { GLB2 }\end{array}$ & $\mathbf{P}$ & $\begin{array}{l}\text { Protective protein for } \\
\text { beta-galactosidase }\end{array}$ & 256540 & S, A & Galactosialidosis (1) & \\
\hline $20 \mathrm{q} 13.11$ & $\mathrm{ADA}$ & C & Adenosine deaminase & 102700 & $\begin{array}{l}\text { S, D, REa, F, } \\
\text { A }\end{array}$ & $\begin{array}{l}\text { Severe combined immunodeficiency } \\
\text { due to ADA deficiency (1); } \\
\text { Hemolytic anemia due to ADA } \\
\text { excess (1) }\end{array}$ & 2(Ada) \\
\hline $20 \mathrm{q} 13.2$ & $\begin{array}{l}\text { GNAS1, } \\
\text { GNAS, } \\
\text { GPSA }\end{array}$ & C & $\begin{array}{l}\text { Guanine nucleotide-binding } \\
\text { protein (G protein), } \\
\text { alpha-stimulating activity } \\
\text { polypeptide-1 }\end{array}$ & 139320 & $\begin{array}{l}\text { REa, H, A, } \\
\text { Fd }\end{array}$ & $\begin{array}{l}\text { Pseudohypoparathyroidism, type Ia } \\
\text { (1); McCune-Albright } \\
\text { polyostotic fibrous dysplasia, } \\
174800 \text { (1); Pituitary tumor, } \\
\text { growth-hormone-secreting (1) }\end{array}$ & 2(Gnas) \\
\hline
\end{tabular}


Table 1-contd

\begin{tabular}{|c|c|c|c|c|c|c|c|}
\hline Location & Symbol & Status & Title & MIM\# & Method & Disorder & Mouse \\
\hline $10 \mathrm{q} 13.2-\mathrm{q} 13.3$ & EBN, BNS & C & Epilepsy, benign neonatal & 121200 & Fd & Epilepsy, benign neonatal (2) & \\
\hline $20 \mathrm{q} 13.2-\mathrm{q} 13.3$ & $\begin{array}{l}\text { FA, FA1, } \\
\text { FACA }\end{array}$ & $\mathbf{P}$ & Fanconi anemia-1 & 227650 & $\mathrm{Fd}$ & Fanconi anemia-1 (2) & \\
\hline Chr.20 & $\begin{array}{l}\text { BMP2, } \\
\text { BMP2A }\end{array}$ & $\mathbf{P}$ & Bone morphogenetic protein-2a & 112261 & H, REa & $\begin{array}{l}\text { ?Fibrodysplasia ossificans } \\
\text { progressiva (1) }\end{array}$ & 2(Bmp2a) \\
\hline $21 \mathrm{q} 11.2$ & MST & L & $\begin{array}{l}\text { Myeloproliferative syndrome, } \\
\text { transient }\end{array}$ & 159595 & $\mathrm{Ch}$ & ?Leukemia, transient (2) & \\
\hline $21 \mathrm{q} 21.3-\mathrm{q} 22.05$ & $\begin{array}{l}\text { APP, AAA, } \\
\text { CVAP }\end{array}$ & C & $\begin{array}{l}\text { Amyloid beta (A4) precursor } \\
\text { protein }\end{array}$ & 104760 & $\begin{array}{l}\text { REa, A, Fd, } \\
\text { RE }\end{array}$ & $\begin{array}{l}\text { Amyloidosis, cerebroarterial, Dutch } \\
\text { type (1); Alzheimer disease, APP } \\
\text { related (3) }\end{array}$ & 16(App) \\
\hline $21 \mathrm{q} 22$ & AML1 & $\mathbf{P}$ & $\begin{array}{l}\text { aml-1 (acute myeloid leukemia) } \\
\text { oncogene }\end{array}$ & 151385 & $\mathrm{Ch}$ & Leukemia, acute myeloid ( 2 ) & \\
\hline $21 \mathrm{q} 22.1-\mathrm{q} 22.2$ & ALS1 & $\mathbf{P}$ & Amyotrophic lateral sclerosis-1 & 105400 & Fd & $\begin{array}{l}\text { Amytrophic lateral sclerosis, one } \\
\text { form (2) }\end{array}$ & \\
\hline $21 q 22.3$ & CBS & C & Cystathionine beta-synthase & 236200 & S, D, A & $\begin{array}{l}\text { Homocystinuria, B6-responsive and } \\
\text { nonresponsive types (1) }\end{array}$ & 17(Cbs) \\
\hline $21 \mathrm{q} 22.3$ & EPM1 & $\mathbf{P}$ & Epilepsy, progressive myoclonic 1 & 254800 & $\mathrm{Fd}$ & $\begin{array}{l}\text { Epilepsy, progressive myoclonus } \\
\text { (2) }\end{array}$ & \\
\hline $21 \mathrm{q} 22.3$ & $\begin{array}{l}\text { ITGB2, } \\
\text { CD18, } \\
\text { LCAMB, } \\
\text { LAD }\end{array}$ & $\mathrm{C}$ & $\begin{array}{l}\text { Integrin, beta-2 (antigen CD18 } \\
\text { (p95), lymphocyte } \\
\text { function-associated antigen-1; } \\
\text { macrophage antigen, beta } \\
\text { polypeptide) }\end{array}$ & 116920 & $\mathrm{~S}, \mathrm{~A}, \mathrm{Fd}$ & Leukocyte adhesion deficiency (1) & $7(\mathrm{Ly}-15)$ \\
\hline $21 \mathrm{q} 22.3$ & PFKL & C & Phosphofructokinase, liver type & 171860 & $S, D, F d$ & $\begin{array}{l}\text { Hemolytic anemia due to } \\
\text { phosphofructokinase deficiency } \\
\text { (1) }\end{array}$ & $17($ Pfkl $)$ \\
\hline 22q11 & CECR, CES & C & Cat eye syndrome & 115470 & Ch, A, D & Cat eye syndrome (2) & \\
\hline 22q11 & DGCR, DGS & C & DiGeorge syndrome & 188400 & Ch, D & DiGeorge syndrome (2) & \\
\hline $22 \mathrm{q} 11$ & HCF2, HC2 & C & Heparin cofactor II & 142360 & REb, REa & $\begin{array}{l}\text { Thrombophilia due to heparin } \\
\text { cofactor II deficiency (1) }\end{array}$ & \\
\hline $22 \mathrm{q} 11$ & NAGA & C & $\begin{array}{l}\text { Acetylgalactosaminidase, alpha-N- } \\
\text { (alpha-galactosidase B) }\end{array}$ & 104170 & S, Ch & Alpha-NAGA deficiency (1) & \\
\hline 22q11 & VCFS & $\mathbf{L}$ & Velocardiofacial syndrome & 192430 & D & ?Velocardiofacial syndrome (2) & \\
\hline $22 \mathrm{q} 11.1-\mathrm{q} 11.2$ & GGT2, GTG & C & Gamma-glutamyltranspeptidase-2 & 231950 & $A, S, F, R E$ & Glutathioninuria (1) & \\
\hline $22 \mathrm{q} 11.2-\mathrm{q} 12.2$ & $\begin{array}{l}\text { CYP2D, } \\
\text { P450C2D }\end{array}$ & C & Cytochrome P450, subfamily IID & 124030 & $\mathrm{~F}$ & $\begin{array}{l}\text { \{?Parkinsonism, susceptibility to\} } \\
\text { (1); Debrisoquine sensitivity (1) }\end{array}$ & 15(Cyp2d) \\
\hline 22q11.2-qter & $\begin{array}{l}\text { SGLT1, } \\
\text { NAGT }\end{array}$ & $\mathbf{P}$ & Sodium-glucose transporter-1 & 182380 & REa & $\begin{array}{l}\text { Glucose/galactose malabsorption } \\
\text { (1) }\end{array}$ & \\
\hline $22 \mathrm{q} 11.2$-qter & TCN2, TC2 & $\mathrm{C}$ & Transcobalamin II & 275350 & F, S, D & Transcobalamin II deficiency (1) & $11(T \mathrm{cn}-2)$ \\
\hline $22 \mathrm{q} 11.21$ & $\begin{array}{l}\text { BCR, CML, } \\
\text { PHL }\end{array}$ & C & $\begin{array}{l}\text { Chronic myeloid leukemia; } \\
\text { breakpoint cluster region }\end{array}$ & 151410 & Ch, RE & Leukemia, chronic myeloid ( 3 ) & \\
\hline $22 \mathrm{q} 11.21-\mathrm{q} 13.1$ & $\mathrm{NF} 2, \mathrm{ACN}$ & C & Acoustic neuroma & 101000 & RE, F & Acoustic neuroma (2) & \\
\hline 22q12 & ES & C & Ewing sarcoma (neuroepithelioma) & 133450 & $\mathrm{Ch}$ & $\begin{array}{l}\text { Ewing sarcoma (2); } \\
\text { Neuroepithelioma (2) }\end{array}$ & \\
\hline 22q12.3-q13.1 & PDGFB, SIS & C & $\begin{array}{l}\text { Platelet-derived growth factor, beta } \\
\text { polypeptide (oncogene SIS) }\end{array}$ & 190040 & $\mathrm{REa}, \mathrm{Fd}$ & Meningioma (3) & 15(Pdgfb) \\
\hline 22q12.3-qter & $\begin{array}{l}\text { MGCR, } \\
\text { MGM }\end{array}$ & C & Meningioma & 156100 & Ch, RE, D & Meningioma (2) & \\
\hline 22q13-qter & ACR, ACRS & $\mathbf{P}$ & Acrosin (proacrosin) & 102480 & REa & $\begin{array}{l}\text { ?Male infertility due to acrosin } \\
\text { deficiency (2) }\end{array}$ & 15 (Acr) \\
\hline $22 q 13.1$ & ADSL, ADS & $\mathbf{P}$ & $\begin{array}{l}\text { Adenylosuccinase (adenylosuccinate } \\
\text { lyase) }\end{array}$ & 103050 & $\mathrm{~S}, \mathrm{REa}$ & Adenylosuccinase deficiency (1) & \\
\hline 22q13.31-qter & ARSA & C & Arylsulfatase A & 250100 & S, D & Metachromatic leukodystrophy (1) & 15(As-2) \\
\hline 22q13.31-qter & DIAl & C & $\begin{array}{l}\text { NADH-diaphorase-1 (cytochrome } \\
\text { b5 reductase) }\end{array}$ & 250800 & S, REa & $\begin{array}{l}\text { Methemoglobinemia, enzymopathic } \\
\text { (1) }\end{array}$ & 15(Dia-1) \\
\hline Xpter-p21 & CSF2RA & $\mathbf{P}$ & $\begin{array}{l}\text { Colony-stimulating factor-2 } \\
\text { receptor, alpha, low-affinity } \\
\text { (granulocyte-macrophage) }\end{array}$ & 306250 & A & $\begin{array}{l}\text { Leukemia, acute myeloid, M2 type } \\
\text { (1) }\end{array}$ & \\
\hline $\mathrm{Xp} 22.32$ & $\begin{array}{l}\text { STS, ARSC1, } \\
\text { SSDD }\end{array}$ & C & Steroid sulfatase, microsomal & 308100 & F, S, D & $\begin{array}{l}\text { Ichthyosis, X-linked (3); Placental } \\
\text { steroid sulfatase deficiency (3) }\end{array}$ & $\mathrm{X}, \mathrm{Y}$ (Sts) \\
\hline $\mathrm{Xp} 22.31$ & $\begin{array}{l}\text { DHOF, } \\
\text { FODH }\end{array}$ & $\mathbf{P}$ & Focal dermal hypoplasia & 305600 & $\mathrm{Ch}$ & Focal dermal hypoplasia (2) & \\
\hline $\mathrm{Xp} 22.3$ & $\begin{array}{l}\text { CDPX1, } \\
\text { CDPXR }\end{array}$ & $\mathrm{C}$ & $\begin{array}{l}\text { Chondrodysplasia punctata, } \\
\mathrm{X} \text {-linked recessive }\end{array}$ & 302950 & $\mathrm{D}, \mathrm{Fd}$ & $\begin{array}{l}\text { Chondrodysplasia punctata, } \\
\text { X-linked recessive (2) }\end{array}$ & \\
\hline $\mathrm{Xp} 22.3$ & $\begin{array}{l}\text { KAL, KMS, } \\
\text { ADMLX }\end{array}$ & C & Kallmann syndrome & 308700 & $\begin{array}{l}\text { F, Fd, D, } \\
\text { REa, REn }\end{array}$ & Kallmann syndrome $(2)$ & \\
\hline $\mathrm{Xp} 22.3$ & OAl & C & $\begin{array}{l}\text { Ocular albinism, Nettleship-Falls } \\
\text { type }\end{array}$ & 300500 & $\mathrm{~F}, \mathrm{Fd}$ & $\begin{array}{l}\text { Ocular albinism, Nettleship-Falls } \\
\text { type (2) }\end{array}$ & \\
\hline $\mathrm{Xp22.3-p22.1}$ & $\begin{array}{l}\text { AMELX, } \\
\text { AMG, } \\
\text { AIH1, } \\
\text { AMGX }\end{array}$ & C & $\begin{array}{l}\text { Amelogenin (amelogenesis } \\
\text { imperfecta, hypoplastic type I) }\end{array}$ & 301200 & $\mathrm{REa}, \mathrm{A}, \mathrm{Fd}$ & Amelogenesis imperfecta (1) & X(Amel) \\
\hline Xp22.3-p21.1 & NHS & C & $\begin{array}{l}\text { Nance-Horan cataract-dental } \\
\text { syndrome }\end{array}$ & 302350 & Fd & Nance-Horan syndrome (2) & ?X(Xcat) \\
\hline $\mathrm{Xp} 22.3-\mathrm{p} 21.1$ & POLA & C & Polymerase, DNA directed, alpha & 312040 & $\mathrm{~S}$ & ?N syndrome, 310465 (1) & X(Pola) \\
\hline $\mathrm{Xp22.3-p22.1}$ & RS & C & Retinoschisis & 312700 & F, Fd & Retinoschisis (2) & \\
\hline $\mathrm{Xp} 22.2$ & CMTX2 & $\mathbf{P}$ & $\begin{array}{l}\text { Charcot-Marie-Tooth disease, } \\
\text { X-linked-2, recessive }\end{array}$ & 302801 & Fd & $\begin{array}{l}\text { Charcot-Marie-Tooth neuropathy, } \\
\text { X-linked-2, recessive (2) }\end{array}$ & \\
\hline $\mathrm{Xp} 22.2-\mathrm{p} 21.3$ & ZFX & C & Zinc finger protein, $\mathrm{X}$-linked & 314980 & $\mathrm{Ch}, \mathrm{REa}, \mathrm{Fd}$ & $46, X Y$ female $(2)$ & $X(Z f x)$ \\
\hline $\mathrm{Xp} 22.2-\mathrm{p} 21.2$ & KFSD & $\mathbf{P}$ & $\begin{array}{l}\text { Keratosis follicularis spinulosa } \\
\text { decalvans }\end{array}$ & 308800 & Fd & $\begin{array}{l}\text { Keratosis follicularis spinulosa } \\
\text { decalvans (2) }\end{array}$ & \\
\hline $\mathrm{Xp} 22.2-\mathrm{p} 22.1$ & CLS & $\mathbf{P}$ & Coffin-Lowry syndrome & 303600 & Fd & Coffin-Lowry syndrome (2) & \\
\hline $\mathrm{Xp} 22.2-\mathrm{p} 22.1$ & $\begin{array}{l}\text { HYP, } \\
\text { HPDR 1 }\end{array}$ & C & Hereditary hypophosphatemia & 307800 & Fd & Hypophosphatemia, hereditary (2) & X(Hyp) \\
\hline $\mathrm{Xp} 22.2-\mathrm{p} 22.1$ & MRXS1 & $\mathbf{P}$ & $\begin{array}{l}\text { Mental retardation, } X \text {-linked, } \\
\text { syndromic-1, with dystonic } \\
\text { movements, ataxia, and seizures }\end{array}$ & 309510 & Fd & $\begin{array}{l}\text { Mental retardation, } \mathrm{X} \text {-linked, } \\
\text { syndromic-1, with dystonic } \\
\text { movements, ataxia, and seizures } \\
(2)\end{array}$ & \\
\hline
\end{tabular}


Table 1-contd

\begin{tabular}{|c|c|c|c|c|c|c|c|}
\hline Location & Symbol & Status & Title & MIM\# & Method & Disorder & Mouse \\
\hline $\mathrm{Xp} 22.2-\mathrm{p} 22.1$ & $\begin{array}{l}\text { PDHA1, } \\
\text { PHE1A }\end{array}$ & C & $\begin{array}{l}\text { Pyruvate dehydrogenase, E1-alpha } \\
\text { polypeptide-1 }\end{array}$ & 312170 & REa, A & $\begin{array}{l}\text { Pyruvate dehydrogenase deficiency } \\
\text { (1) }\end{array}$ & $\mathrm{X}$ (Pdha-1) \\
\hline Xp22.2-p22.1 & $\begin{array}{l}\text { PHK, } \\
\text { PHKA2 }\end{array}$ & $\mathbf{P}$ & $\begin{array}{l}\text { Phosphorylase kinase deficiency, } \\
\text { liver (glycogen storage disease } \\
\text { type VIII) }\end{array}$ & 306000 & Fd, REa & $\begin{array}{l}\text { Glycogen storage disease, } \mathrm{X} \text {-linked } \\
\text { hepatic (2) }\end{array}$ & \\
\hline $\mathrm{Xp} 22$ & $\begin{array}{l}\text { AGMX2, } \\
\text { XLA2, } \\
\text { IMD6 }\end{array}$ & $\mathbf{P}$ & $\begin{array}{l}\text { Agammaglobulinemia, } \mathrm{X} \text {-linked } 2 \\
\text { (with growth hormone } \\
\text { deficiency) }\end{array}$ & 300310 & $\mathrm{Fd}$ & $\begin{array}{l}\text { Agammaglobulinemia, type } 2 \text {, } \\
\text { X-linked (2) }\end{array}$ & $\mathrm{X}(\mathrm{Xid})$ \\
\hline $\mathrm{Xp} 22$ & AIC & $\mathrm{C}$ & Aicardi syndrome & 304050 & $\mathrm{X} / \mathrm{A}, \mathrm{Ch}$ & Aicardi syndrome (2) & \\
\hline $\mathrm{Xp} 22$ & GY & $\mathbf{L}$ & $\begin{array}{l}\text { Hereditary hypophosphatemia II } \\
\text { (gyro equivalent) }\end{array}$ & 307810 & $\mathrm{H}$ & $\begin{array}{l}\text { ?Hypophosphatemia with deafness } \\
\text { (2) }\end{array}$ & $X(G y)$ \\
\hline $\mathrm{Xp} 22$ & $\begin{array}{l}\text { HOMG, HSH, } \\
\text { HMGX }\end{array}$ & $\mathbf{L}$ & $\begin{array}{l}\text { Hypomagnesemia, } \mathrm{X} \text {-linked } \\
\text { primary }\end{array}$ & 307600 & $\mathrm{X} / \mathrm{A}$ & $\begin{array}{l}\text { Hypomagnesemia, } \mathrm{X} \text {-linked } \\
\text { primary (2) }\end{array}$ & \\
\hline $\mathrm{Xp} 22$ & MRX1 & $\mathrm{C}$ & $\begin{array}{l}\text { Mental retardation, } \mathrm{X} \text {-linked-1, } \\
\text { non-dysmorphic }\end{array}$ & 309530 & $\mathrm{~F}, \mathrm{Fd}, \mathrm{D}$ & $\begin{array}{l}\text { Mental retardation, X-linked-1, } \\
\text { non-dysmorphic (2) }\end{array}$ & \\
\hline $\mathrm{Xp} 22$ & $\begin{array}{l}\text { SEDL, } \\
\text { SEDT }\end{array}$ & C & Spondyloepiphyseal dysplasia tarda & 313400 & Fd & $\begin{array}{l}\text { Spondyloepiphyseal dysplasia tarda } \\
\text { (2) }\end{array}$ & \\
\hline $\mathrm{Xp22-p21}$ & $\begin{array}{l}\text { GDXY, } \\
\text { TDFX }\end{array}$ & $\mathbf{P}$ & $\begin{array}{l}\text { Gonadal dysgenesis, } \mathrm{XY} \text { female } \\
\text { type }\end{array}$ & 306100 & $\mathrm{~F}, \mathrm{Ch}$ & $\begin{array}{l}\text { Gonadal dysgenesis, XY female } \\
\text { type (2) }\end{array}$ & \\
\hline $\mathrm{Xp} 21.3-\mathrm{p} 21.1$ & RP3 & C & $\begin{array}{l}\text { Retinitis pigmentosa-3 (RP with } \\
\text { metallic sheen in heterozygotes) }\end{array}$ & 312610 & Fd, D & Retinitis pigmentosa-3 (2) & \\
\hline $\mathrm{Xp} 21.3-\mathrm{p} 21.2$ & AHC, AHX & C & Primary adrenal hypoplasia & 300200 & $\mathrm{D}, \mathrm{Fd}$ & Adrenal hypoplasia, primary (2) & \\
\hline $\mathrm{Xp} 21.3-\mathrm{p} 21.2$ & GK & C & Glycerol kinase & 307030 & $\mathrm{D}, \mathrm{Fd}$ & Glycerol kinase deficiency (2) & \\
\hline $\mathrm{Xp} 21.2$ & $\mathrm{DMD}, \mathrm{BMD}$ & C & $\begin{array}{l}\text { Dystrophin (muscular dystrophy, } \\
\text { Duchenne and Becker types) }\end{array}$ & 310200 & $\mathrm{X} / \mathrm{A}, \mathrm{Fd}, \mathrm{D}$ & $\begin{array}{l}\text { Duchenne muscular dystrophy (3); } \\
\text { Becker muscular dystrophy (3); } \\
\text { Cardiomyopathy, dilated, } \\
\text { X-linked (3) }\end{array}$ & $\mathrm{X}(\mathrm{Dmd})$ \\
\hline $\mathrm{Xp} 21.2-\mathrm{p} 21.1$ & $\mathrm{XK}$ & C & Xk blood group & 314850 & F, D & [McLeod phenotype] (2) & \\
\hline $\mathrm{Xp} 21.1$ & CYBB, CGD & C & Chronic granulomatous disease & 306400 & F, D & $\begin{array}{l}\text { Chronic granulomatous disease, } \\
\text { X-linked (3) }\end{array}$ & $\mathrm{X}(\mathrm{Cybb})$ \\
\hline Xp21.1 & OTC & C & Ornithine transcarbamylase & 311250 & L, REa, A, D & $\begin{array}{l}\text { Ornithine transcarbamylase } \\
\text { deficiency (3) }\end{array}$ & $X($ spf; Otc) \\
\hline Xp21.1-p11.3 & $\begin{array}{l}\text { COD1, } \\
\text { PCDX }\end{array}$ & $\mathbf{P}$ & $\begin{array}{l}\text { Progressive cone dystrophy, } \\
\text { X-linked }\end{array}$ & 304020 & $\mathrm{Fd}$ & Progressive cone dystrophy (2) & \\
\hline Xp21.1-q22 & MRXS6 & $\mathbf{P}$ & $\begin{array}{l}\text { Mental retardation, } \mathrm{X} \text {-linked, } \\
\text { syndromic-6, with gynecomastia } \\
\text { and obesity }\end{array}$ & 309585 & Fd & $\begin{array}{l}\text { Mental retardation, } \mathrm{X} \text {-linked, } \\
\text { syndromic-6, with gynecomastia } \\
\text { and obesity (2) }\end{array}$ & \\
\hline $\mathrm{Xp} 21$ & GTD & $\mathrm{L}$ & Gonadotropin deficiency & 306190 & $\mathrm{D}$ & $\begin{array}{l}\text { ?Gonadotropin deficiency (2); } \\
\text { ?Cryptorchidism (2) }\end{array}$ & \\
\hline Xp21-p11 & THC & $\mathbf{P}$ & Thrombocytopenia, X-linked & 313900 & Fd & Thrombocytopenia, X-linked (2) & \\
\hline $\mathrm{Xp} 11.4$ & NDP, ND & C & Norrie disease & 310600 & Fd, D & Norrie disease (2) & \\
\hline Xp11.4-p11.23 & PFC, PFD & C & $\begin{array}{l}\text { Properdin P factor, complement } \\
\text { (properdin P deficiency) }\end{array}$ & 312060 & Fd, REa, A & Properdin deficiency, X-linked (3) & $X(P f c)$ \\
\hline Xp11.3 & CSNB1 & $\mathbf{I}$ & $\begin{array}{l}\text { Congenital stationary } \\
\text { nightblindness }\end{array}$ & 310500 & Fd & $\begin{array}{l}\text { Nightblindness, congenital } \\
\text { stationary, type I ( } 2 \text { ) }\end{array}$ & \\
\hline Xp11.3 & RP2 & C & Retinitis pigmentosa-2 & 312600 & $\mathbf{F d}$ & Retinitis pigmentosa-2 (2) & \\
\hline $\mathrm{Xp} 11.3-\mathrm{p} 11.2$ & WAS, IMD2 & C & Wiskott-Aldrich syndrome & 301000 & $\mathrm{Fd}, \mathrm{X} / \mathrm{A}$ & Wiskott-Aldrich syndrome (2) & \\
\hline $\mathrm{Xp} 11.23$ & $\begin{array}{l}\text { GF1, ERYF1, } \\
\text { NFE1 }\end{array}$ & C & Globin transcription factor-1 & 305371 & $\mathrm{REa}, \mathrm{A}$ & $\begin{array}{l}\text { ?Hereditary persistence of fetal } \\
\text { hemoglobin, Swiss type (2) }\end{array}$ & $\mathrm{X}(\mathrm{Gf}-1)$ \\
\hline $\mathrm{Xp} 11.21$ & $\begin{array}{l}\text { ALAS2, ASB, } \\
\text { ANH1 }\end{array}$ & $\mathrm{C}$ & Aminolevulinate, delta-, synthase- 2 & 301300 & $\mathrm{Ch}, \mathrm{REa}, \mathrm{A}$ & $\begin{array}{l}\text { Anemia, sideroblastic/hypochromic } \\
\text { (3) }\end{array}$ & \\
\hline $\mathrm{Xp} 11.21$ & IP1, IP & C & $\begin{array}{l}\text { Incontinentia pigmenti-1, sporadic } \\
\text { type }\end{array}$ & 308300 & $\mathrm{X} / \mathrm{A}$ & $\begin{array}{l}\text { Incontinentia pigmenti, sporadic } \\
\text { type (2) }\end{array}$ & $\mathbf{X}(\mathbf{T d})$ \\
\hline $\mathrm{Xp} 11.2$ & SSRC & $\mathbf{P}$ & Sarcoma, synovial & 312820 & $\mathrm{Ch}, \mathrm{RE}$ & Sarcoma, synovial (2) & \\
\hline $\mathrm{Xp} 11-\mathrm{q} 11$ & AIED, OA2 & C & $\begin{array}{l}\text { Aland island eye disease (ocular } \\
\text { albinism, Forsius-Eriksson type) }\end{array}$ & 300600 & $\mathrm{~F}, \mathrm{D}, \mathrm{Fd}$ & $\begin{array}{l}\text { Ocular albinism, Forsius-Eriksson } \\
\text { type (2) }\end{array}$ & \\
\hline $\mathrm{Xp} 11-\mathrm{q} 21$ & MRXS2 & $\mathbf{P}$ & $\begin{array}{l}\text { Mental retardation, X-linked, } \\
\text { syndromic-2, with dysmorphism } \\
\text { and cerebral atrophy; Prieto } \\
\text { syndrome }\end{array}$ & 309610 & Fd & $\begin{array}{l}\text { Mental retardation, } \mathrm{X} \text {-linked, } \\
\text { syndromic-2, with dysmorphism } \\
\text { and cerebral atrophy }(2)\end{array}$ & \\
\hline Xp11-q21.3 & MRXS3 & $\mathbf{P}$ & $\begin{array}{l}\text { Mental retardation, } \mathrm{X} \text {-linked, } \\
\text { syndromic-3, with spastic diplegia }\end{array}$ & 309470 & $\mathrm{Fd}$ & $\begin{array}{l}\text { Mental retardation, } \mathrm{X} \text {-linked, } \\
\text { syndromic-3, with spastic } \\
\text { diplegia }(2)\end{array}$ & \\
\hline $\mathrm{Xp}$ & CCT & $\mathbf{L}$ & Cataracts, congenital total & 302200 & $\mathrm{Fd}$ & ?Cataract, congenital total (2) & \\
\hline $\mathrm{Xp}$ & RTS & $\bar{L}$ & Rett syndrome & 312750 & $\mathrm{Ch}$ & ?Rett syndrome (2) & \\
\hline Xcen-q21.3 & SGB & L & Simpson-Galabi-Behmel syndrome & 312870 & $\mathrm{Fd}$ & $\begin{array}{l}\text { ?Simpson-Galabi-Behmel } \\
\text { syndrome (2) }\end{array}$ & \\
\hline Xcen-q22 & $\begin{array}{l}\text { AR, DHTR, } \\
\text { TFM, } \\
\text { SBMA, } \\
\text { KD, } \\
\text { SMAX1 }\end{array}$ & $\mathrm{C}$ & $\begin{array}{l}\text { Androgen receptor (testicular } \\
\text { feminization) }\end{array}$ & 313700 & $\mathrm{~S}, \mathrm{Fd}, \mathrm{REa}, \mathrm{A}$ & $\begin{array}{l}\text { Testicular feminization (1); } \\
\text { Reifenstein syndrome (1); } \\
\text { Infertile male syndrome (1); } \\
\text { Spinal and bulbar muscular } \\
\text { atrophy of Kennedy, } 313200 \text { (3) }\end{array}$ & $X(T f m)$ \\
\hline $\mathrm{Xq} 11-\mathrm{q} 12$ & $\mathrm{MRX} 2$ & $\mathbf{L}$ & $\begin{array}{l}\text { Mental retardation, } \mathrm{X} \text {-linked-2, } \\
\text { non-dysmorphic }\end{array}$ & 309540 & $\mathbf{F d}$ & $\begin{array}{l}\text { Mental retardation, X-linked-2, } \\
\text { non-dysmorphic }(2)\end{array}$ & \\
\hline $\mathrm{Xq12-q13}$ & MNK, MK & C & Menkes disease & 309400 & Fc, $X / A, H$ & Menkes disease (2) & $\mathbf{X}(\mathbf{M o})$ \\
\hline Xq12-q13 & PHKA1 & $\mathbf{P}$ & $\begin{array}{l}\text { Phosphorylase kinase, muscle, } \\
\text { alpha polypeptide }\end{array}$ & 311870 & $\mathrm{REa}, \mathrm{A}$ & ?Muscle glycogenosis (1) & X(Phka) \\
\hline$X q 12-q 21.1$ & DYT3 & $\mathbf{P}$ & $\begin{array}{l}\text { Torsion dystonia-parkinsonism, } \\
\text { Filipino type }\end{array}$ & 314250 & Fd & $\begin{array}{l}\text { Torsion dystonia-parkinsonism, } \\
\text { Filipino type (2) }\end{array}$ & \\
\hline $\mathrm{Xq} 12.2-13.1$ & EDA, HED & C & Anhidrotic ectodermal dysplasia & 305100 & $\mathrm{X} / \mathrm{A}, \mathrm{H}, \mathrm{Fd}$ & Anhidrotic ectodermal dysplasia (2) & $\mathrm{X}(\mathrm{Ta})$ \\
\hline $\mathrm{Xq13}$ & $\begin{array}{l}\text { CMTX1, } \\
\text { CMT2 }\end{array}$ & C & $\begin{array}{l}\text { Charcot-Marie-Tooth disease, } \\
\text { X-linked-1, dominant }\end{array}$ & 302800 & Fd & $\begin{array}{l}\text { Charcot-Marie-Tooth neuropathy, } \\
\text { X-linked-1, dominant (2) }\end{array}$ & \\
\hline $\mathrm{Xq13}$ & FGDY, AAS & $\mathbf{P}$ & $\begin{array}{l}\text { Aarskog-Scott syndrome } \\
\text { (faciogenital dysplasia) }\end{array}$ & 305400 & $\mathbf{X} / \mathbf{A}$ & Aarskog-Scott syndrome (2) & \\
\hline $\mathrm{Xq13}$ & $\begin{array}{l}\text { PGK1, } \\
\text { PGKA }\end{array}$ & C & Phosphoglycerate kinase-1 & 311800 & $\begin{array}{l}\mathrm{S}, \mathrm{R}, \mathrm{REb} \\
\mathrm{Fd}\end{array}$ & $\begin{array}{l}\text { Hemolytic anemia due to PGK } \\
\text { deficiency (1); Myoglobinuria/ } \\
\text { hemolysis due to PGK deficiency } \\
\text { (1) }\end{array}$ & $\mathrm{X}(\mathrm{Pgk}-1)$ \\
\hline
\end{tabular}


Table 1-contd

\begin{tabular}{|c|c|c|c|c|c|c|c|}
\hline Location & Symbol & Status & Title & MIM\# & Method & Disorder & Mouse \\
\hline $\mathrm{Xq13-q21}$ & WWS & $\mathbf{P}$ & Wieacker-Wolff syndrome & 314580 & Fd & Wieacker-Wolff syndrome (2) & \\
\hline $\mathrm{Xq} 13-\mathrm{q} 21.1$ & DFN3 & C & $\begin{array}{l}\text { Conductive deafness with stapes } \\
\text { fixation }\end{array}$ & 304400 & Fd, D & $\begin{array}{l}\text { Deafness, conductive, with stapes } \\
\text { fixation ( } 2 \text { ) }\end{array}$ & \\
\hline Xq13-q21.31 & CPX & C & Cleft palate, $\mathrm{X}$-linked & 303400 & Fd, D & Cleft palate, X-linked (2) & \\
\hline Xq13-q22 & MRXS4 & $\mathbf{P}$ & $\begin{array}{l}\text { Mental retardation, X-linked, } \\
\text { syndromic- } 4 \text {, with congenital } \\
\text { contractures and low fingertip } \\
\text { arches }\end{array}$ & 309605 & $\mathrm{Fd}$ & $\begin{array}{l}\text { Mental retardation, } \mathrm{X} \text {-linked, } \\
\text { syndromic-4, with congenital } \\
\text { contractures and low fingertip } \\
\text { arches }(2)\end{array}$ & \\
\hline $\mathrm{Xq} 13.1$ & $\begin{array}{l}\text { RPS4X, } \\
\text { CCG2, } \\
\text { SCAR }\end{array}$ & C & Ribosomal protein S4, X-linked & 312760 & A, REa & Turner syndrome (1) & $X(\operatorname{Rps} 4 x)$ \\
\hline $\mathrm{Xq} 13.1-\mathrm{q} 21.1$ & $\begin{array}{l}\text { SCIDX1, } \\
\text { SCIDX, } \\
\text { IMD4 }\end{array}$ & C & $\begin{array}{l}\text { Severe combined } \\
\text { immunodeficiency, } \mathrm{X} \text {-linked }\end{array}$ & 300400 & Fd & $\begin{array}{l}\text { Severe combined } \\
\text { immunodeficiency, X-linked ( } 2 \text { ) }\end{array}$ & \\
\hline $\mathrm{Xq} 21$ & AHDS, AHS & $\mathbf{P}$ & $\begin{array}{l}\text { Allan-Herndon mental retardation } \\
\text { syndrome }\end{array}$ & 309600 & $\mathrm{Fd}$ & Allan-Herndon syndrome (2) & \\
\hline $\mathrm{Xq} 21-\mathrm{q} 22$ & $\begin{array}{l}\text { SPG2, } \\
\text { SPPX2 }\end{array}$ & $\mathbf{P}$ & $\begin{array}{l}\text { Spastic paraplegia, } \mathrm{X} \text {-linked, } \\
\text { uncomplicated }\end{array}$ & 312920 & $\mathrm{Fd}$ & $\begin{array}{l}\text { Spastic paraplegia, } X \text {-linked, } \\
\text { uncomplicated }(2)\end{array}$ & \\
\hline $\mathrm{Xq} 21-\mathrm{q} 22$ & TBG & $\mathbf{P}$ & Thyroxine-binding globulin & 314200 & REa, A & $\begin{array}{l}\text { [Euthyroidal hyper- and } \\
\text { hypothyroxinemia] (1) }\end{array}$ & \\
\hline $\mathrm{Xq} 21.2$ & CHM, TCD & $\mathrm{C}$ & Choroideremia & 303100 & $\begin{array}{l}\text { Fd, LD, D, } \\
\quad \text { A, Ch, X/A }\end{array}$ & Choroideremia (2) & \\
\hline $\mathrm{Xq} 21.3-\mathrm{q} 22$ & $\begin{array}{l}\text { AGMX1, } \\
\text { IMD1, } \\
\text { XLA }\end{array}$ & C & $\begin{array}{l}\text { Agammaglobulinemia, X-linked } 1 \\
\text { (Bruton) }\end{array}$ & 300300 & $\mathrm{H}, \mathrm{Fd}$ & $\begin{array}{l}\text { Agammaglobulinemia, type } 1 \text {, } \\
\text { X-linked (2) }\end{array}$ & \\
\hline $\mathrm{Xq} 21.3-\mathrm{q} 22$ & $\begin{array}{l}\text { MGC1, } \\
\text { MGCN }\end{array}$ & $\mathbf{P}$ & Megalocornea, X-linked & 309300 & $\mathrm{Fd}$ & Megalocornea, X-linked (2) & \\
\hline $\mathrm{Xq} 21.3-\mathrm{q} 22$ & PHP, GHDX & $\mathbf{L}$ & $\begin{array}{l}\text { Growth hormone deficiency, } \\
\text { X-linked (panhypopituitarism, } \\
\text { X-linked) }\end{array}$ & 312000 & $\mathrm{Fd}$ & $\begin{array}{l}\text { ?Growth hormone deficiency, } \\
\text { X-linked (2) }\end{array}$ & \\
\hline $\mathrm{Xq} 22$ & $\begin{array}{l}\text { COL4A5, } \\
\text { ATS, ASLN }\end{array}$ & C & Collagen IV, alpha-5 polypeptide & 303630 & $\mathrm{REa}, \mathrm{A}, \mathrm{Fd}$ & Alport syndrome, 301050 (3) & \\
\hline $\mathrm{Xq} 22$ & GLA & C & Galactosidase, alpha & 301500 & $\mathrm{~S}, \mathrm{R}, \mathrm{A}, \mathrm{Fd}$ & Fabry disease (3) & X(Ags) \\
\hline $\mathrm{Xq} 22$ & PLP, PMD & $\mathrm{C}$ & $\begin{array}{l}\text { Proteolipid protein; } \\
\text { Pelizaeus-Merzbacher disease }\end{array}$ & 312080 & $\begin{array}{l}\text { REa, A, Ch, } \\
\text { R, Fd }\end{array}$ & Pelizaeus-Merzbacher disease ( 3 ) & $\mathrm{X}(\mathrm{Plp}(\mathrm{jp}))$ \\
\hline $\mathrm{Xq} 22-\mathrm{q} 24$ & PRPS1 & C & $\begin{array}{l}\text { Phosphoribosylpyrophosphate } \\
\text { synthetase I }\end{array}$ & 311850 & $\mathrm{~S}, \mathrm{R}, \mathrm{REa}, \mathrm{A}$ & $\begin{array}{l}\text { Phosphoribosylpyrophosphate } \\
\text { synthetase-related gout (1) }\end{array}$ & \\
\hline $\mathrm{Xq} 24-\mathrm{q} 27$ & $\begin{array}{l}\text { HIGM1, } \\
\text { IMD3 }\end{array}$ & $\mathbf{P}$ & $\begin{array}{l}\text { X-linked immunodeficiency with } \\
\text { hyper-IgM }\end{array}$ & 308230 & Fd & $\begin{array}{l}\text { Immunodeficiency, X-linked, with } \\
\text { hyper-IgM (2) }\end{array}$ & \\
\hline $\mathrm{Xq} 25$ & $\begin{array}{l}\text { LYP, IMD5, } \\
\quad \text { XLP, XLPD }\end{array}$ & C & $\begin{array}{l}\text { Lymphoproliferative syndrome, } \\
\text { X-linked }\end{array}$ & 308240 & Fd, D & $\begin{array}{l}\text { Lymphoproliferative syndrome, } \\
\text { X-linked (2) }\end{array}$ & \\
\hline $\mathrm{Xq} 25-\mathrm{q} 27$ & $\begin{array}{l}\text { PGS, } \\
\text { MRXS5 }\end{array}$ & $\mathbf{P}$ & $\begin{array}{l}\text { Pettigrew syndrome (mental } \\
\text { retardation, X-linked, with } \\
\text { Dandy-Walker malformation, } \\
\text { basal ganglia disease, and } \\
\text { seizures) }\end{array}$ & 304340 & $\mathrm{Fd}$ & $\begin{array}{l}\text { Mental retardation, } \mathrm{X} \text {-linked, } \\
\text { syndromic-5, with } \\
\text { Dandy-Walker malformation, } \\
\text { basal ganglia disease, and } \\
\text { seizures (2) }\end{array}$ & \\
\hline $\mathrm{Xq} 26-\mathrm{q} 27$ & BFLS & $\mathbf{P}$ & $\begin{array}{l}\text { Borjeson-Forssman-Lehmann } \\
\text { syndrome }\end{array}$ & 301900 & Fd & $\begin{array}{l}\text { Borjeson-Forssman-Lehmann } \\
\text { syndrome (2) }\end{array}$ & \\
\hline $\mathrm{Xq} 26-\mathrm{q} 27$ & $\begin{array}{l}\text { HPT, HPTX, } \\
\text { HYPX }\end{array}$ & $\mathbf{P}$ & Hypoparathyroidism, X-linked & 307700 & $\mathrm{Fd}$ & Hypoparathyroidism, X-linked (2) & \\
\hline $\mathrm{Xq} 26-\mathrm{q} 27$ & POF1, POF & L & Premature ovarian failure & 311360 & $\mathrm{Ch}$ & Ovarian failure, premature (2) & \\
\hline $\mathrm{Xq} 26-\mathrm{q} 27.2$ & HPRT & $\mathrm{C}$ & $\begin{array}{l}\text { Hypoxanthine-guanine } \\
\text { phosphoribosyltransferase }\end{array}$ & 308000 & $\begin{array}{l}\mathrm{S}, \mathrm{M}, \mathrm{C}, \mathrm{R} \\
\mathrm{REa}, \mathrm{Fd}\end{array}$ & $\begin{array}{l}\text { Lesch-Nyhan syndrome (3); } \\
\text { HPRT-related gout (1) }\end{array}$ & $\mathrm{X}(\mathrm{Hprt})$ \\
\hline $\mathrm{Xq} 26.1$ & $\begin{array}{l}\text { OCRL, } \\
\text { LOCR }\end{array}$ & $\mathrm{C}$ & Lowe oculocerebrorenal syndrome & 309000 & $\mathrm{X} / \mathrm{A}, \mathrm{Fd}$ & Lowe syndrome (2) & \\
\hline $\mathrm{Xq} 26.3-\mathrm{q} 27.1$ & $\begin{array}{l}\text { ADFN, } \\
\text { ALDS }\end{array}$ & $\mathbf{P}$ & Albinism-deafness syndrome & 300700 & Fd & Albinism-deafness syndrome (2) & \\
\hline $\mathrm{Xq} 27-\mathrm{q} 28$ & ANOP1 & $\mathbf{L}$ & $\begin{array}{l}\text { Anophthalmos-1 (with mental } \\
\text { retardation but without } \\
\text { anomalies) }\end{array}$ & 301590 & $\mathbf{F}$ & ?Anophthalmos-1 (2) & \\
\hline $\mathrm{Xq} 27-\mathrm{q} 28$ & IP2 & $\mathbf{P}$ & $\begin{array}{l}\text { Incontinentia pigmenti-2 (familial, } \\
\text { male-lethal type) }\end{array}$ & 308310 & $\mathrm{Fd}$ & Incontinentia pigmenti, familial ( 2 ) & $\mathrm{X}(? \mathrm{Str})$ \\
\hline $\mathrm{Xq} 27.1-\mathrm{q} 27.2$ & F9, HEMB & $\mathrm{C}$ & $\begin{array}{l}\text { Coagulation factor IX (hemophilia } \\
\text { B) }\end{array}$ & 306900 & $\begin{array}{l}\mathrm{REa}, \mathrm{A}, \mathrm{Fd} \\
\mathrm{D}, \mathrm{X} / \mathrm{A}, \mathrm{RE}\end{array}$ & Hemophilia B (3) & $X(C f-9)$ \\
\hline $\mathrm{Xq} 27.3$ & $\begin{array}{l}\text { FRAXA, } \\
\text { FMR1 }\end{array}$ & $\mathrm{C}$ & Fragile site $\mathrm{Xq} 27.3$ & 309550 & $\begin{array}{l}\mathrm{Ch}, \mathrm{F}, \mathrm{Fd} \\
\mathrm{RE}\end{array}$ & Martin-Bell syndrome (2) & $\mathrm{X}(\mathrm{Fmr}-1)$ \\
\hline $\mathrm{Xq} 28$ & ALD & $\mathrm{C}$ & Adrenoleukodystrophy & 300100 & F, Fd, D & $\begin{array}{l}\text { Adrenoleukodystrophy (2); } \\
\text { Adrenomyeloneuropathy (2) }\end{array}$ & \\
\hline Xq28 & CBBM, BCM & C & $\begin{array}{l}\text { Blue-monochromatic } \\
\text { colorblindness (blue cone } \\
\text { monochromacy) }\end{array}$ & 303700 & $\mathrm{~F}, \mathrm{Fd}, \mathrm{RE}$ & $\begin{array}{l}\text { Colorblindness, blue } \\
\text { monochromatic (3) }\end{array}$ & \\
\hline $\mathrm{Xq} 28$ & $\begin{array}{l}\text { CDPX2, } \\
\text { CPXD, } \\
\text { CPX }\end{array}$ & $\mathbf{L}$ & $\begin{array}{l}\text { Chondrodysplasia punctata, } \\
\text { X-linked dominant (Happle } \\
\text { syndrome) }\end{array}$ & 302960 & $\mathbf{H}$ & $\begin{array}{l}\text { Chondrodysplasia punctata, } \\
\text { X-linked dominant (2) }\end{array}$ & $\mathrm{X}(\mathrm{Bpa})$ \\
\hline $\mathrm{Xq28}$ & $\begin{array}{l}\text { DIR, DI1, } \\
\text { ADHR }\end{array}$ & $\mathrm{C}$ & $\begin{array}{l}\text { Nephrogenic diabetes insipidus } \\
\text { (vasopressin V2-receptor) }\end{array}$ & 304800 & Fd, S, REa & Diabetes insipidus, nephrogenic (3) & \\
\hline $\mathrm{Xq} 28$ & DKC & $\mathbf{P}$ & Dyskeratosis congenita & 305000 & Fd & Dyskeratosis congenita (2) & \\
\hline $\mathrm{Xq} 28$ & EFE2, BTHS & $\mathrm{C}$ & $\begin{array}{l}\text { Endocardial fibroelastosis-2 (Barth } \\
\text { syndrome; cardioskeletal } \\
\text { myopathy with neutropenia and } \\
\text { abnormal mitochondria) }\end{array}$ & 302060 & $\mathrm{Fd}$ & Endocardial fibroelastosis-2 (2) & \\
\hline $\mathrm{Xq} 28$ & EMD & $\mathrm{C}$ & $\begin{array}{l}\text { Emery-Dreifuss muscular } \\
\text { dystrophy }\end{array}$ & 310300 & $\mathrm{~F}, \mathrm{Fd}, \mathrm{H}$ & $\begin{array}{l}\text { Emery-Dreifuss muscular } \\
\text { dystrophy (2) }\end{array}$ & \\
\hline $\mathrm{Xq} 28$ & F8C, HEMA & $\mathrm{C}$ & $\begin{array}{l}\text { Coagulation factor VIII } \\
\text { (hemophilia A) }\end{array}$ & 306700 & $\begin{array}{l}\text { F, Fd, REa, } \\
\text { A, RE }\end{array}$ & Hemophilia A (3) & $X(C f-8)$ \\
\hline $\mathrm{Xq} 28$ & $\begin{array}{l}\text { G6PD, } \\
\text { G6PD1 }\end{array}$ & $\mathrm{C}$ & $\begin{array}{l}\text { Glucose-6-phosphate } \\
\text { dehydrogenase }\end{array}$ & 305900 & $\begin{array}{l}\mathrm{F}, \mathrm{S}, \mathrm{REb} \\
\mathrm{RE}\end{array}$ & $\begin{array}{l}\text { G6PD deficiency (3); Favism (1); } \\
\text { Hemolytic anemia due to G6PD } \\
\text { deficiency (1) }\end{array}$ & $X(G 6 p d)$ \\
\hline
\end{tabular}


Table 1-contd

\begin{tabular}{|c|c|c|c|c|c|c|c|}
\hline Location & Symbol & Status & Title & MIM\#\# & Method & Disorder & Mouse \\
\hline $\mathrm{Xq} 28$ & GCP, CBD & $\mathrm{C}$ & $\begin{array}{l}\text { Deutan colorblindness (green cone } \\
\text { pigment) }\end{array}$ & 303800 & $\mathrm{~F}, \mathrm{RE}, \mathrm{A}, \mathrm{Fd}$ & Colorblindness, deutan ( 3 ) & $X(R s v p)$ \\
\hline $\mathrm{Xq} 28$ & $\begin{array}{c}\text { HSAS1, } \\
\text { HSAS, } \\
\text { HYCX }\end{array}$ & $\mathrm{C}$ & $\begin{array}{l}\text { Hydrocephalus, } \mathrm{X} \text {-linked, due to } \\
\text { stenosis of aqueduct of Sylvius }\end{array}$ & 307000 & $\mathrm{Fd}$ & Hydrocephalus, X-linked (2) & \\
\hline $\mathrm{Xq} 28$ & $\begin{array}{l}\text { IDS, MPS2, } \\
\text { SIDS }\end{array}$ & $\mathrm{C}$ & $\begin{array}{l}\text { Hunter syndrome (sulfoiduronate } \\
\text { sulfatase deficiency) }\end{array}$ & 309900 & $\begin{array}{l}\mathrm{X} / \mathrm{A}, \mathrm{Fd}, \mathrm{F}, \\
\mathrm{RE}\end{array}$ & Mucopolysaccharidosis II (2) & $\mathrm{X}(\mathrm{Ids})$ \\
\hline $\mathrm{Xq} 28$ & $\begin{array}{l}\text { MAFD2, } \\
\text { MDX }\end{array}$ & $\mathrm{L}$ & Manic-depressive illness, $\mathrm{X}$-linked & 309200 & $\mathrm{~F}$ & $\begin{array}{l}\text { ?Manic-depressive illness, X-linked } \\
\text { (2) }\end{array}$ & \\
\hline $\mathrm{Xq} 28$ & MASA & $\mathrm{C}$ & $\begin{array}{l}\text { MASA syndrome (complicated } \\
\text { spastic paraplegia) }\end{array}$ & 303350 & $\mathrm{Fd}$ & MASA syndrome (2) & \\
\hline $\mathrm{Xq} 28$ & $\begin{array}{l}\text { MRSD, } \\
\text { CHRS }\end{array}$ & $\mathbf{P}$ & $\begin{array}{l}\text { Mental retardation-skeletal } \\
\text { dysplasia }\end{array}$ & 309620 & $\mathrm{Fd}$ & $\begin{array}{l}\text { Mental retardation-skeletal } \\
\text { dysplasia }(2)\end{array}$ & \\
\hline $\mathrm{Xq} 28$ & MRX3 & $\mathbf{L}$ & Mental retardation, $\mathrm{X}$-linked-3 & 309541 & $\mathrm{Fd}$ & ?Mental retardation, X-linked-3 (2) & \\
\hline $\mathrm{Xq} 28$ & $\begin{array}{l}\text { MTM1, } \\
\text { MTMX }\end{array}$ & $\mathrm{C}$ & Myotubular myopathy, X-linked & 310400 & $\mathrm{Fd}$ & $\begin{array}{l}\text { Myotubular myopathy, X-linked } \\
\text { (2) }\end{array}$ & \\
\hline $\mathrm{Xq} 28$ & MYP1, BED & $P$ & Myopia-1 (Bornholm eye disease) & 310460 & $\mathrm{Fd}$ & $\begin{array}{l}\text { Myopia-1 (2); Bornholm eye } \\
\text { disease (2) }\end{array}$ & \\
\hline $\mathrm{Xq} 28$ & OPD1 & $\mathbf{P}$ & Otopalatodigital syndrome, type I & 311300 & $\mathrm{Fd}$ & $\begin{array}{l}\text { Otopalatodigital syndrome, type I } \\
\text { (2) }\end{array}$ & \\
\hline $\mathrm{Xq} 28$ & $\mathrm{RCP}, \mathrm{CBP}$ & $\mathrm{C}$ & $\begin{array}{l}\text { Protan colorblindness (red cone } \\
\text { pigment) }\end{array}$ & 303900 & $\mathrm{~F}, \mathrm{RE}, \mathrm{A}, \mathrm{Fd}$ & Colorblindness, protan (3) & $X(R s v p)$ \\
\hline $\mathrm{Xq} 28$ & TKC, TKCR & $\mathrm{C}$ & Goeminne TKCR syndrome & 314300 & $\mathrm{X} / \mathrm{A}$ & Goeminne TKCR syndrome (2) & \\
\hline $\mathrm{Xq} 28$ & $\begin{array}{l}\text { WSN, } \\
\text { BGMR }\end{array}$ & $\mathbf{P}$ & $\begin{array}{l}\text { Waisman syndrome (basal ganglion } \\
\text { disorder with mental retardation) }\end{array}$ & 311510 & $\mathrm{Fd}$ & $\begin{array}{l}\text { Waisman parkinsonism-mental } \\
\text { retardation syndrome }(2)\end{array}$ & \\
\hline
\end{tabular}

Table 2 The morbid anatomy of the human genome (by disorder).

\begin{tabular}{|c|c|}
\hline Location & Location \\
\hline Aarskog-Scott syndrome (2) & Angelman syndrome $(2) \ldots$ \\
\hline ?Abetalipoproteinemia (1) .. & Angioedema, hereditary (1) ......... \\
\hline [Acanthocytosis, one form] (1) ........ & Anhidrotic ectodermal dysplasia (2). \\
\hline Acatalasemia $(1) \ldots \ldots \ldots \ldots \ldots \ldots \ldots \ldots \ldots$ & Aniridia of WAGR syndrome (2) ... \\
\hline Acetyl-CoA carboxylase deficiency $(1) \ldots \ldots \ldots \ldots \ldots \ldots \ldots \ldots \ldots \ldots, 17 \mathrm{q} 21$ & Aniridia-2 (3) $\ldots \ldots \ldots \ldots \ldots \ldots \ldots$ \\
\hline Acid-maltase deficiency, adult $(1) \ldots \ldots \ldots \ldots \ldots \ldots \ldots \ldots \ldots \ldots \ldots \ldots \ldots \ldots \ldots \ldots \ldots$ & ?Anophthalmos-1 (2) $\ldots \ldots \ldots \ldots \ldots \ldots \ldots \ldots \ldots$ \\
\hline 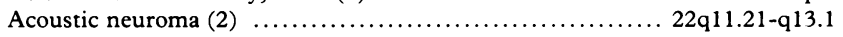 & Anterior segment mesenchymal dysgenesis (2). \\
\hline ACTH deficiency $(1) \ldots \ldots \ldots \ldots \ldots \ldots$ & Antithrombin III deficiency (3) $\ldots \ldots \ldots \ldots \ldots \ldots$ \\
\hline Acyl-CoA dehydrogenase, long chain, & ApoA-I and apoC-III deficiency, combined (1) \\
\hline deficiency of $(1) \ldots \ldots \ldots \ldots \ldots \ldots \ldots$ & Apolipoprotein B-100, defective (1) ............ \\
\hline $\begin{array}{l}\text { Acyl-CoA dehydrogenase, medium chain, } \\
\text { deficiency of }(1) \ldots \ldots \ldots \ldots \ldots \ldots \ldots \ldots\end{array}$ & $\begin{array}{l}\text { [Apolipoprotein } \mathrm{H} \text { deficiency] }(1) \ldots \ldots \\
\text { Argininemia }(1) \ldots \ldots \ldots \ldots \ldots \ldots \ldots \ldots\end{array}$ \\
\hline $\begin{array}{l}\text { Acyl-CoA dehydrogenase, short chain, } \\
\text { deficiency of }(1) \ldots \ldots \ldots \ldots \ldots \ldots \ldots \ldots \ldots\end{array}$ & $\begin{array}{l}\text { Argininosuccinicaciduria (1) } \ldots \ldots \ldots \ldots \ldots \ldots \ldots \ldots \\
\text { Aspartylglucosaminuria }(3) \ldots \ldots \ldots \ldots \ldots \ldots \ldots \ldots\end{array}$ \\
\hline Adenylosuccinase deficiency (1) ................ & Ataxia-telangiectasia $(2) \ldots \ldots \ldots \ldots \ldots \ldots \ldots \ldots$ \\
\hline $\begin{array}{l}\text { Adrenal hyperplasia, congenital, } \\
\text { due to } 11 \text {-beta-hydroxylase deficiency (1) ... }\end{array}$ & $\begin{array}{l}\text { Atherosclerosis, susceptibility to }(2) \ldots \ldots \ldots \ldots \ldots \ldots \\
\text { Atopy }(2) \ldots \ldots \ldots \ldots \ldots \ldots \ldots \ldots \ldots \ldots \ldots \ldots \ldots \ldots \ldots\end{array}$ \\
\hline & 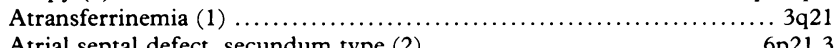 \\
\hline $\begin{array}{l}\text { due to } 21 \text {-hydroxylase def } \\
\text { Adrenal hyperplasia II (1). }\end{array}$ & $\begin{array}{l}\text { Atrial septal defect, secundum type (2) ......... } \\
\text { Autonomic failure due to DBH deficiency (1). }\end{array}$ \\
\hline Adrenal hyperplasia $\mathrm{V}(1) \ldots \ldots \ldots$ & Basal cell nevus syndrome $(2) \ldots \ldots \ldots \ldots \ldots \ldots$ \\
\hline Adrenal hypoplasia, primary (2) .... & Batten disease (2) .................. \\
\hline Adrenocortical carcinoma (2) .... & ?Batten disease, one form, 204200 (1) . \\
\hline phy (2) ... & Becker muscular dystrophy (3) ........ \\
\hline pathy (2) .... & Beckwith-Wiedemann syndrome (2) .. \\
\hline ongenital] (1) $\ldots \ldots \ldots \ldots \ldots$ & 17pter-p12 \\
\hline hia, type $1, \mathrm{X}$-linked (2) ... & ..... Chr.1 \\
\hline mia, type $2, \mathrm{X}$-linked $(2)$ & thus inversus and ptosis (2) \\
\hline (2) $\ldots \ldots \ldots \ldots \ldots \ldots \ldots \ldots$ & Bloom syndrome, $210900(2) \ldots \ldots \ldots \ldots \ldots \ldots \ldots$ \\
\hline adrome (2) & Borjeson-Forssman-Lehmann syndrome (2) \\
\hline Albinism (3) ...................... & Bornholm eye disease $(2) \ldots \ldots \ldots \ldots \ldots \ldots$ \\
\hline 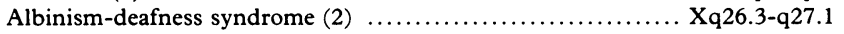 & Branchiootic syndrome (2). \\
\hline 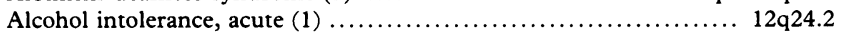 & ?Breast cancer (1) ........... \\
\hline ?Aldolase A deficiency $(1) \ldots \ldots \ldots \ldots \ldots \ldots \ldots \ldots \ldots \ldots \ldots \ldots \ldots \ldots \ldots \ldots \ldots \ldots, 16 q 22-q 24$ & Breast cancer $(1) \ldots \ldots \ldots$ \\
\hline$\ldots \ldots \ldots . . . \times 921$ & reast cancer, ductal (2) \\
\hline sin deficiency $(1) \ldots \ldots \ldots \ldots \ldots \ldots \ldots \ldots \ldots \ldots \ldots$ & ductal $(2) \ldots \ldots \ldots$ \\
\hline 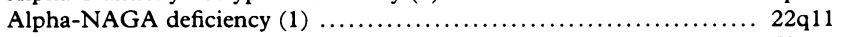 & Breast cancer-1, early onset $(2) \ldots$ \\
\hline (n.......................... & 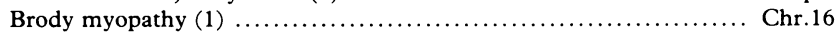 \\
\hline 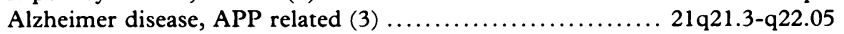 & Burkitt lymphoma $(3) \ldots \ldots \ldots \ldots \ldots \ldots \ldots \ldots \ldots$ \\
\hline 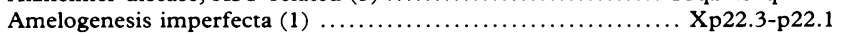 & ?Clq deficiency $(1) \ldots \ldots . .$. \\
\hline deficiency, erythrocyte] $(1) \ldots \ldots \ldots \ldots \ldots \ldots \ldots$ lp21-p13 & ?Clq deficiency $(1) \ldots \ldots \ldots \ldots \ldots$ \\
\hline opathy, familial, & $\mathrm{C} 1 \mathrm{r} / \mathrm{C} 1 \mathrm{~s}$ deficiency, combined (1) \\
\hline & ... $12 \mathrm{p} 13$ \\
\hline type $(1) \ldots \ldots \ldots \ldots \ldots \ldots 21 \mathrm{q} 21.3-\mathrm{q} 22.05$ & C2 deficiency (3) .. \\
\hline Amyloidosis, Finnish type, 105120 (1) .......................... 9q34 & C3 deficiency $(1) \ldots \ldots \ldots \ldots$ \\
\hline 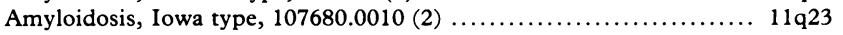 & $\mathrm{C} 3 \mathrm{~b}$ inactivator deficiency $(1)$ \\
\hline idosis, secondary, susceptibility to $\}(1) \ldots \ldots \ldots \ldots \ldots \ldots$ 1q21-q23 & $\mathrm{C} 4$ deficiency $(3) \quad \ldots \ldots \ldots \ldots . .$. \\
\hline 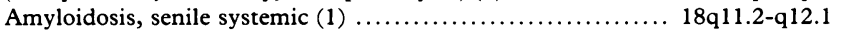 & .. 6p21.3 \\
\hline 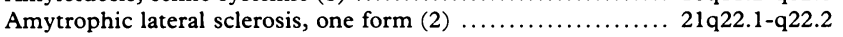 & .. 9q34.1 \\
\hline 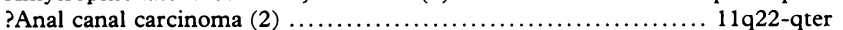 & C6 deficiency (1) \\
\hline ..................... 4q11-q13 & C7 deficiency (1). \\
\hline , due to DHFR & C8 deficiency, type I (2). \\
\hline .. $5 \mathrm{q} 11.2-\mathrm{q} 13.2$ & C8 deficiency, type II (2) \\
\hline & C9 deficiency (1) $\ldots \ldots \ldots$. \\
\hline & $8 \mathrm{q} 21.4$ \\
\hline Anemia, sideroblastic/hypochromic ( 3 ) & Carbamoylphosphate synthetase I deficiency $(1) \ldots \ldots \ldots \ldots \ldots$. \\
\hline Aneurysm, familial (1) .. & 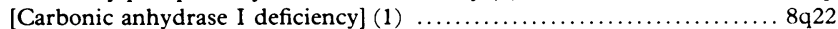 \\
\hline
\end{tabular}


Table 2-contd

Disorder

Location

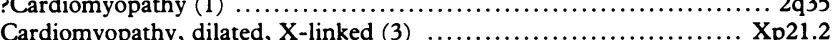

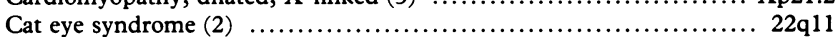

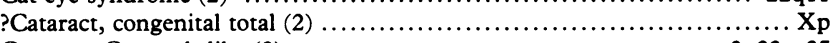

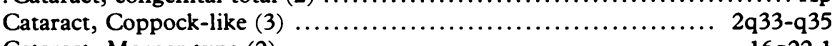

Cataract, Marner type (2) .................................... 16q22.1

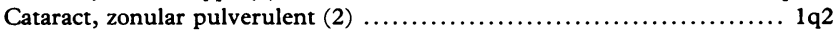

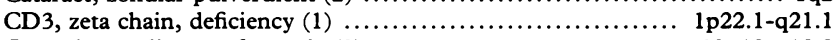

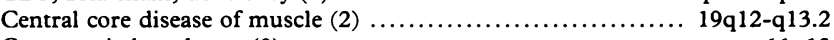

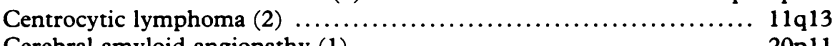

Cerebral amyloid angiopathy (1)

20p11

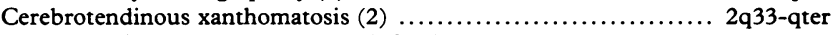

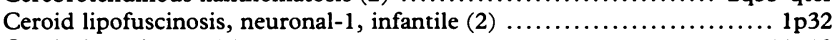

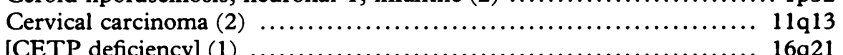

Charcot-Marie-Tooth neuropathy,

slow nerve conduction type Ia $(2)$

Charcot-Marie-Tooth neuropathy,

slow nerve conduction type Ib (2)

$17 \mathrm{p} 11.2$

Charcot-Marie-Tooth neuropathy,

$\mathrm{X}$-linked-1, dominant (2)

$1 \mathrm{q} 21.2-\mathrm{q} 23$

Charcot-Marie-Tooth neuropathy,

$\mathrm{X}$-linked-2, recessive (2)

$\mathrm{Xq} 13$

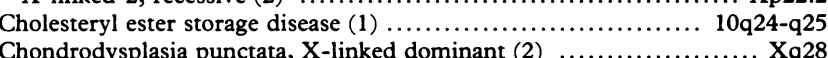

$\mathrm{Xp} 22.2$

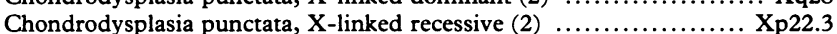

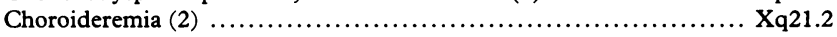

Chronic granulomatous disease, autosomal,

due to deficiency of CYBA (3)

Chronic granulomatous disease

due to deficiency of NCF-1 (1)

$16 \mathrm{q} 24$

Chronic granulomatous disease

due to deficiency of NCF-2 (1)

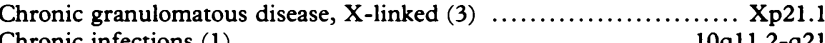

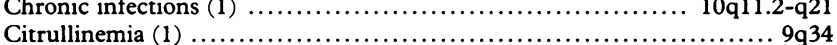

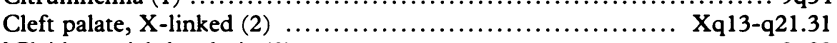

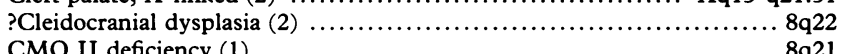

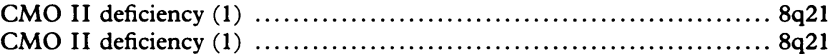

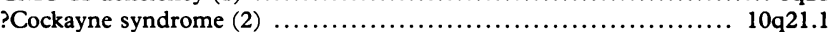

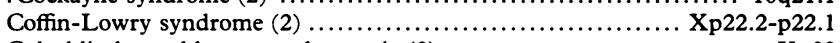

Colorblindness, blue monochromatic (3) . ...................... Xq28

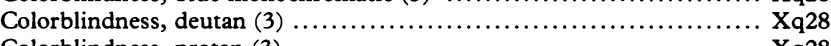

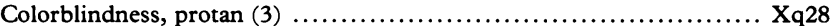

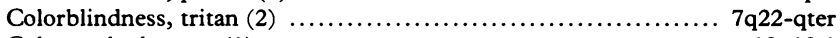

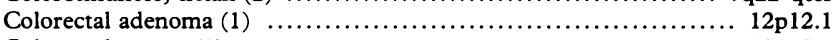

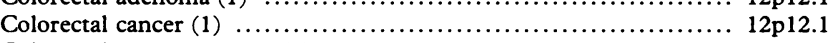

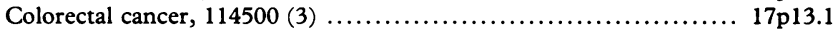

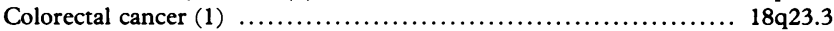

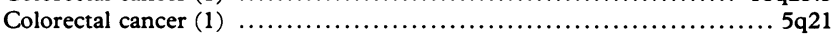

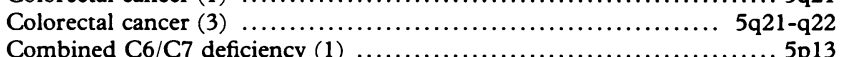

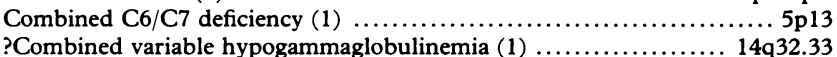

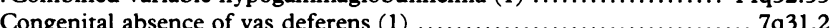

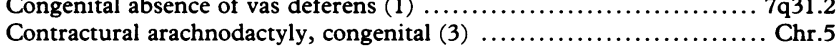

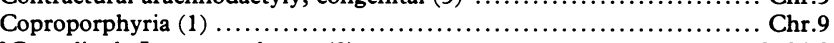

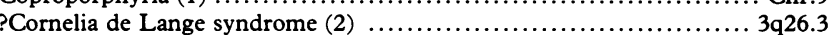

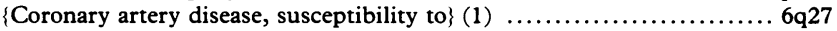

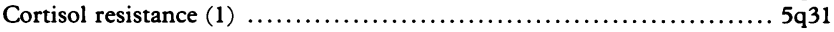

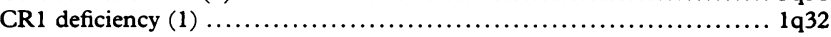

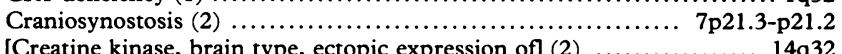

[Creatine kinase, brain type, ectopic expression of] (2) $\ldots \ldots \ldots \ldots \ldots \ldots \ldots \ldots \ldots \ldots$ 14q32

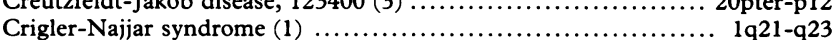

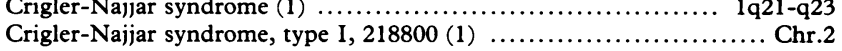

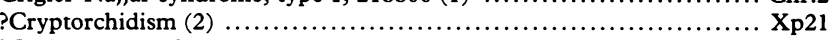

?Cutis laxa, marfanoid neonatal type (1) ................. 7q31.1-q31.3

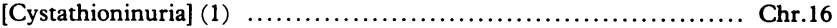

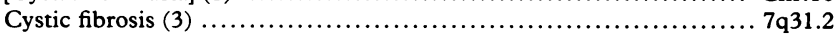

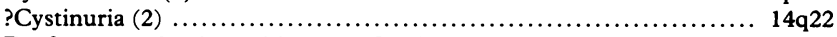

Deafness, conductive, with stapes fixation (2) ................ Xq13-q21.1

Deafness, low-tone (2) .................................. 5q31-q33

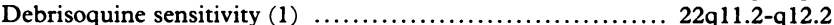

Dentinogenesis imperfecta-1 (2) ........................... 4q13-q21

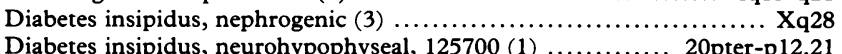

Diabetes insipidus, neurohypophyseal, 125700 (1) .......... 20pter-p12.21

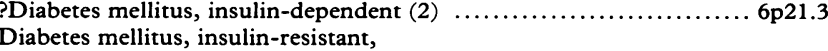

Diabetes mellitus, insulin-resistant,

with acanthosis nigricans $(1)$
Diabetes mellitus, rare form $(1) \ldots \ldots \ldots \ldots \ldots \ldots \ldots \ldots \ldots \ldots \ldots \ldots \ldots \ldots \ldots \ldots \ldots \ldots \ldots \ldots \ldots \ldots \ldots \ldots \ldots \ldots \ldots \ldots \ldots \ldots$
$11 \mathrm{p} 15.5$

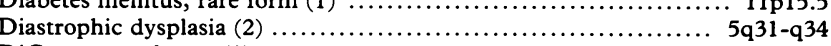

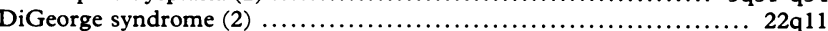

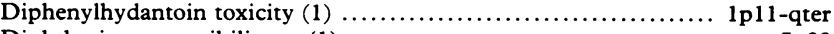

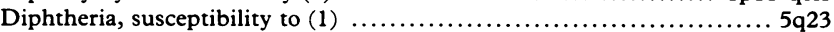

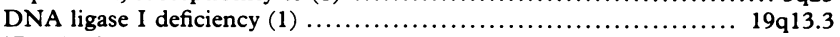

?Dubin-Johnson syndrome $(2)$
Duchenne muscular dystrophy $(3) \ldots \ldots \ldots \ldots \ldots \ldots \ldots \ldots \ldots \ldots \ldots \ldots \ldots \ldots \ldots \ldots \ldots \ldots \ldots \ldots \ldots \ldots \ldots \ldots \ldots \ldots \ldots \ldots \ldots$

[Dysalbuminemic hyperthyroxinemia] (1) $\ldots \ldots \ldots \ldots \ldots \ldots \ldots \ldots, 4 q \ldots \ldots \ldots \ldots$

[Dysalbuminemic hyperzincemia] (1) ....................... 4q11-q13

Disorder

Location

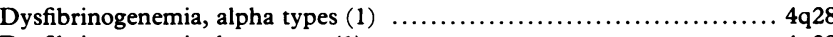

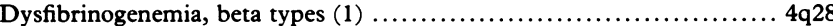

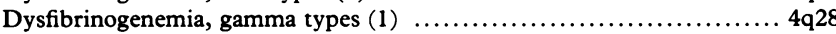

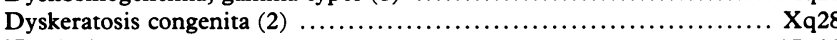

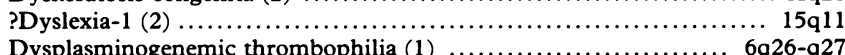

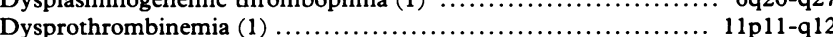

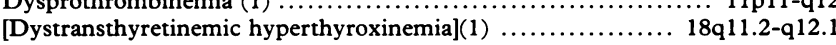

?EEC syndrome (2) .................................. 7q11.2-q21.3

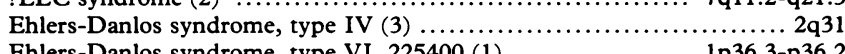

Ehlers-Danlos syndrome, type VIIA1 (3) .............. 17q21.31-q22.05

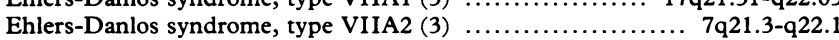

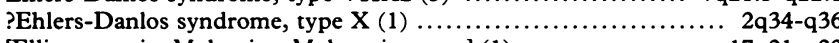

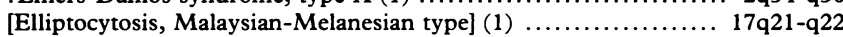

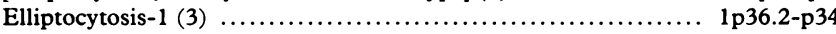

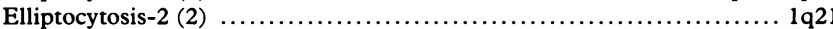

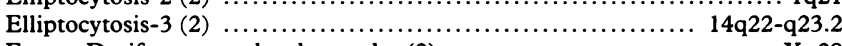

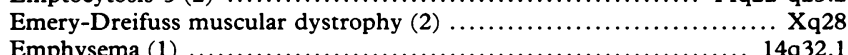

Emphysema due to alpha-2-macroglobulin

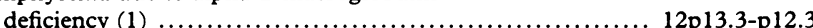

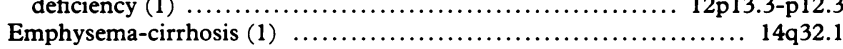

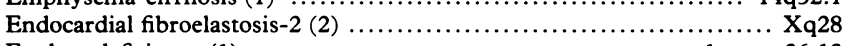

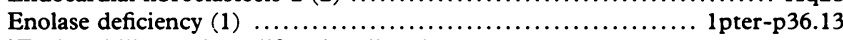

?Eosinophilic myeloproliferative disorder (2) .................. 12p13

Epidermolysis bullosa dystrophica, dominant, $131750(3) \ldots \ldots \ldots \ldots \ldots 3 \mathrm{p} 21$

Epidermolysis bullosa dystrophica, recessive, 226600 (3) $\ldots \ldots \ldots \ldots \ldots \ldots .3 \mathrm{p} 21$

Epidermolysis bullosa, Ogna type $(2) \ldots \ldots \ldots \ldots \ldots \ldots \ldots \ldots \ldots \ldots \ldots \ldots \ldots \ldots \ldots \ldots \ldots \ldots$
Epidermolysis bullosa simplex, $131900(3) \ldots \ldots \ldots \ldots \ldots \ldots \ldots \ldots$

Epidermolysis bullosa simplex,

Dowling-Meara type, 131760 (3) ..................... 12q11-q13

Epidermolysis bullosa simplex,

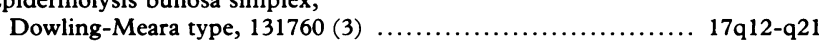

Epidermolysis bullosa simplex, generalized,

$131900(1) \ldots \ldots \ldots \ldots \ldots \ldots \ldots \ldots \ldots \ldots \ldots \ldots \ldots \ldots$
?Epidermolysis bullosa simplex, localized,

$131800(1)$

$12 \mathrm{q} 11-\mathrm{q} 13$

?Epidermolysis bullosa, Weber Cockayne type,

$131800(2)$

$12 \mathrm{q} 11-\mathrm{q} 13$

Epidermolytic hyperkeratosis, 113800 (1)

Epidermolytic hyperkeratosis, 113800 (2)

Epilepsy, benign neonatal (2)

Epilepsy, juvenile myoclonic (2)

Epilepsy, progressive myoclonus (2)

Epithelioma, self-healing, squamous 1,

Ferguson-Smith type (2)

?Erythremia (1)

$12 \mathrm{q} 11-\mathrm{q} 13$

$17 \mathrm{q} 21-\mathrm{q} 22$
$12 \mathrm{q} 11-\mathrm{q} 13$

Erythremias, alpha- (1)

..6 621.3

Erythremias, beta- (1)

$21 \mathrm{q} 22.3$

Erythroblastosis fetalis (1)

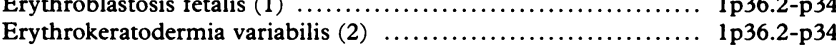

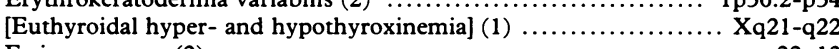

Ewing sarcoma (2)

Exertional myoglobinuria

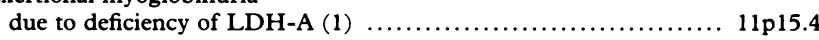

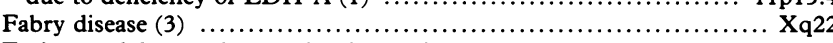

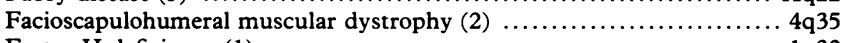

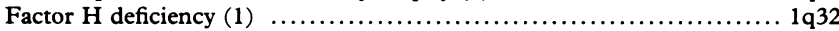

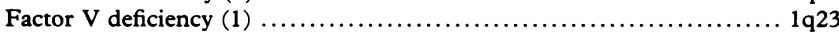

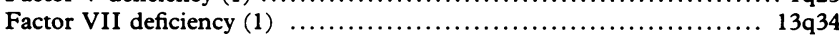

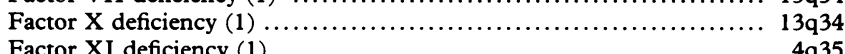

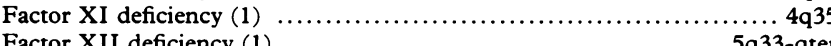

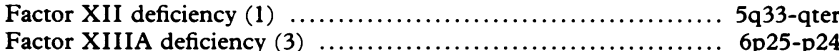

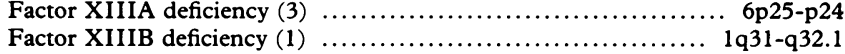

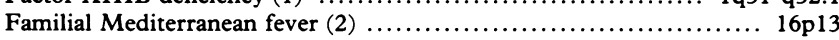

?Familial Mediterranean fever, 249100 (1) $\ldots \ldots \ldots \ldots \ldots \ldots \ldots \ldots$ 9q32-q33

?Fanconi anemia (1)

Fanconi anemia-1 (2) ....................................... 20. $13.2-\mathrm{q} 13.3$

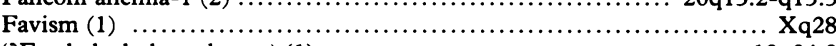

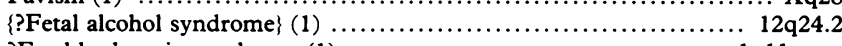

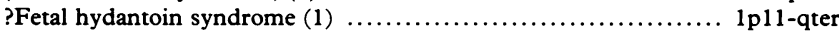

?Fibrodysplasia ossificans progressiva (1) $\ldots \ldots \ldots \ldots \ldots \ldots \ldots . .20$

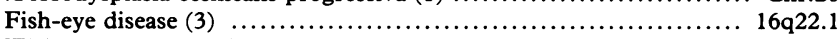

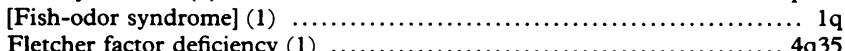

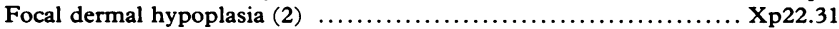

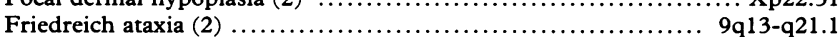

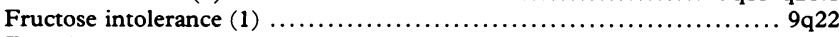

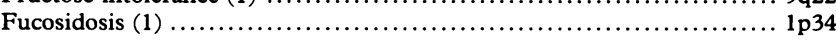

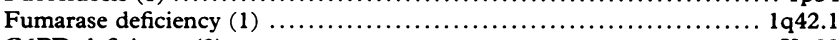

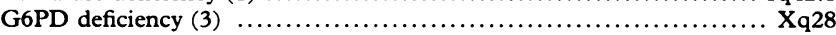

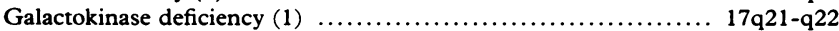

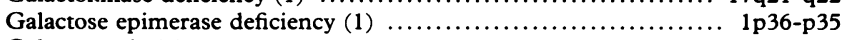

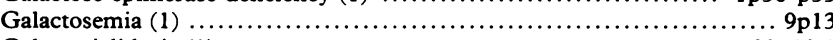

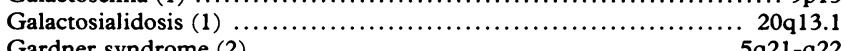

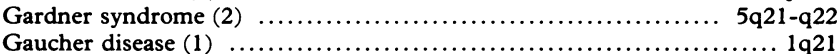

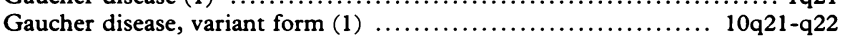


Table 2-contd

Disorder

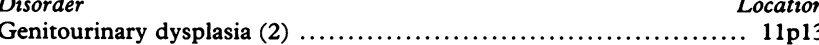

Gerstmann-Straussler disease, 137440 (3) $\ldots \ldots \ldots \ldots \ldots \ldots \ldots \ldots$ 20pter-p12

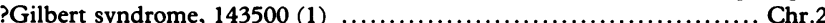

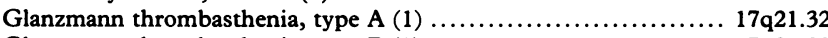

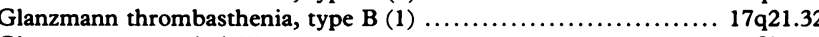

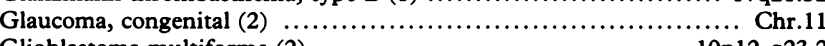

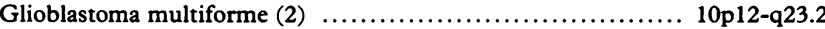

Glucose/galactose malabsorption (1) ..................... 22q11.2-qter

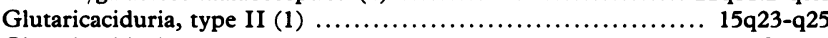

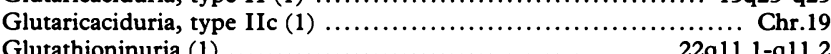

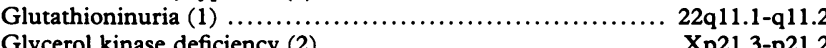

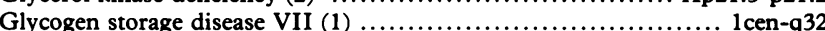

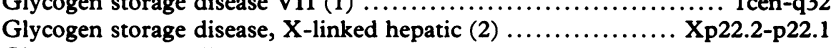

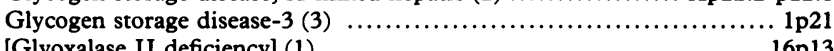

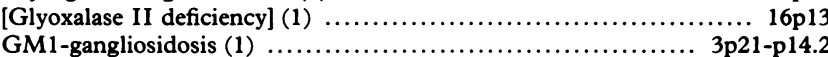

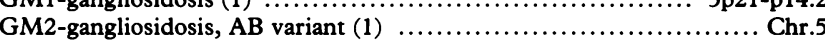

GM2-gangliosidosis, juvenile, adult (1) ...................... 15q23-q24

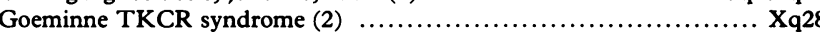

Goiter, adolescent multinodular (1) $\ldots \ldots \ldots \ldots \ldots \ldots \ldots \ldots \ldots \ldots$ 8q24.2-q24.3

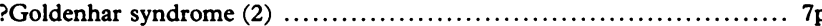

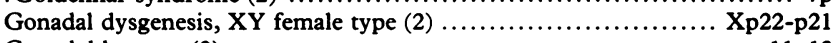

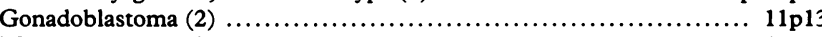

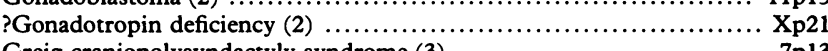

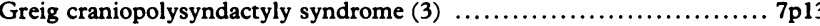

?Growth hormone deficiency, X-linked (2) ................. Xq21.3-q22

?Gynecomastia, familial, due to increased

aromatase activity (1)

$15 \mathrm{q} 21.1$

Gyrate atrophy of choroid and retina with ornithinemia

B6 responsive or unresponsive (1)

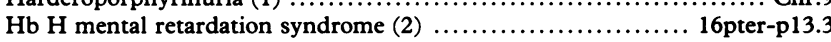

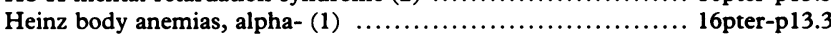

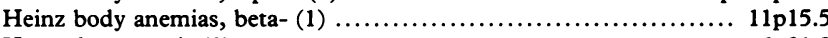

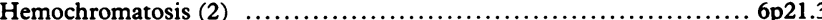

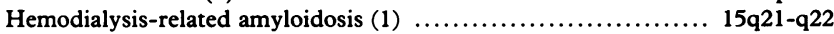

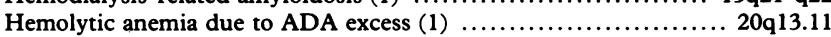

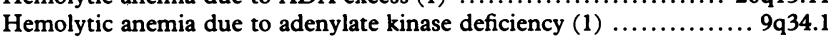

Hemolytic anemia due to bisphosphoglycerate

mutase deficiency (1)

$7 q 31-q 34$

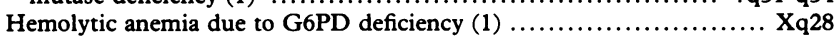

Hemolytic anemia due to glucosephosphate

isomerase deficiency (1)

Hemolytic anemia due to glutathione

peroxidase deficiency (1)

19 cen-q12

$3 q 11-q 12$

Hemolytic anemia due to glutathione

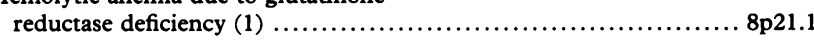

Hemolytic anemia due to hexokinase deficiency $(1) \ldots \ldots \ldots \ldots \ldots \ldots . \ldots \ldots$ 10q22

Hemolytic anemia due to PGK deficiency $(1) \ldots \ldots \ldots \ldots \ldots \ldots \ldots \ldots \ldots \ldots \ldots$

Hemolytic anemia due to phosphofructol

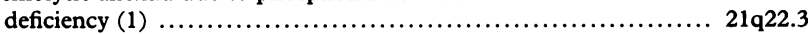

Hemolytic anemia due to triosephosphate isomerase

deficiency (1)

$12 \mathrm{p} 13$

Hemophilia A (3)

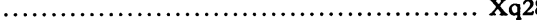

Hemophilia B (3) ................................ Xq27.1-q27.2

Hemorrhagic diathesis due to 'antithrombin'

Pittsburgh (1)

$\mathrm{Xq27.1-q27.2}$

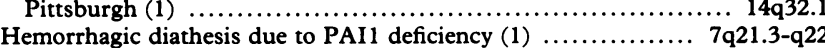

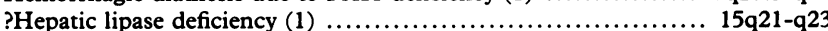

?Hepatocarcinoma (1) .................................... 2q14-q21

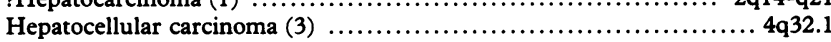

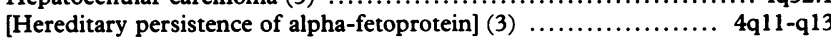

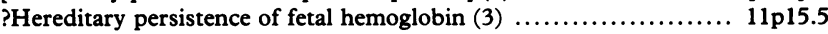

?Hereditary persistence of fetal hemoglobin,

heterocellular, Indian type (2)

$7 \mathrm{q} 36$

?Hereditary persistence of fetal hemoglobin,

Swiss type (2)

Xp11.23

Hers disease, or glycogen storage disease VI (1) .................. Chr.14

Heterocellular hereditary persistence of

fetal hemoglobin (2)

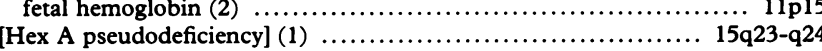

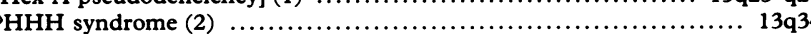

[Histidinemia] (1) ...................................... 12q22-q23

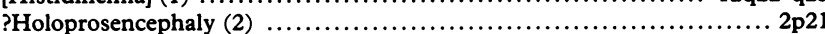

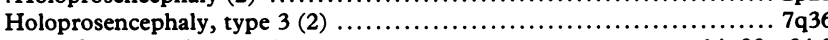

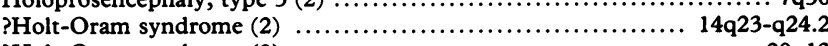

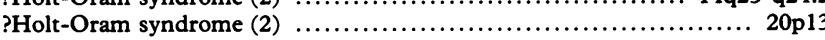

Homocystinuria, B6-responsive and

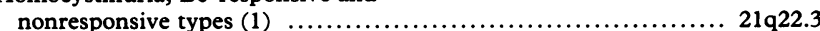

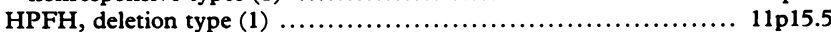

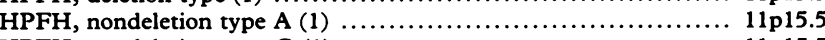

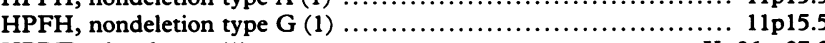

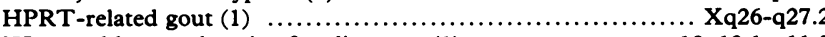

?Humoral hypercalcemia of malignancy (1) $\ldots \ldots \ldots \ldots \ldots \ldots$ 12p12.1-p11.2

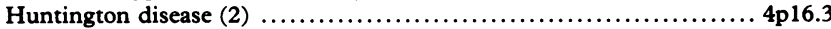

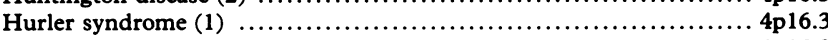

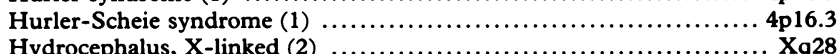

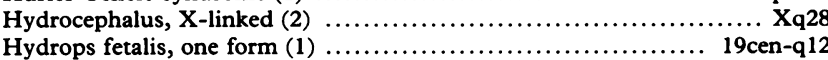

Disorder

Location

3-hydroxyacyl-CoA dehydrogenase deficiency (1) $\ldots \ldots \ldots \ldots \ldots \ldots \ldots . . \ldots \ldots h r .7$

Hyperammonemia due to CTPase deficiency (1) .............. 1p13-p11

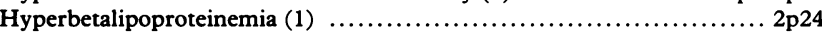

Hypercalcemia, hypocalciuric, familial (2) ................. 3q21-q24

Hypercholesterolemia, familial (3) ..................... 19p13.2-p13.1

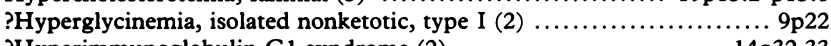

?Hyperimmunoglobulin $G 1$ syndrome $(2) \ldots \ldots \ldots \ldots \ldots \ldots \ldots 14 \mathrm{q} 32.33$

Hyperkalemic periodic paralysis (3) ....................17q23.1-q25.3

?Hyperleucinemia-isoleucinemia or hypervalinemia (1) .......... 12pter-q12

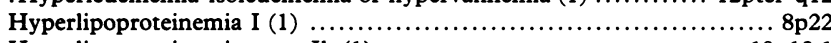

Hyperlipoproteinemia, type Ib (1) ............................. 19q13.1

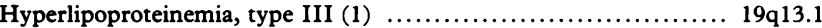

[Hyperphenylalaninemia, mild] (3) ............................ 12q24.1

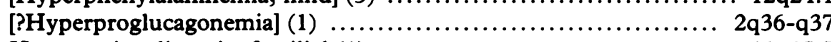

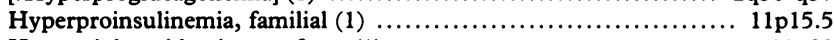

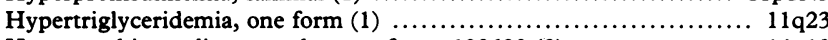

Hypertrophic cardiomyopathy, one form, 192600 (3) ............. 14q12

?Hypervalinemia or hyperleucine-isoleucinemia (1) ............... Chr.19

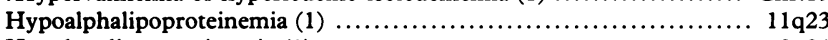

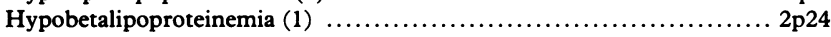

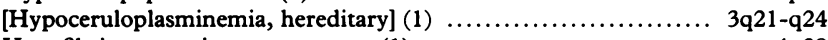

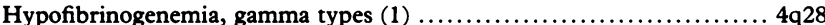

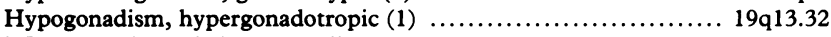

?Hypogonadotropic hypogonadism

due to GNRH deficiency, 227200 (1) .................. 8p21-p11.2

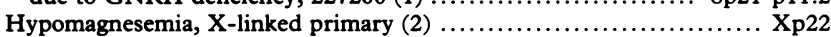

?Hypomelanosis of Ito (2) .............................. 15q11-q13

?Hypomelanosis of Ito (2) ................................. 9q 33 -qter

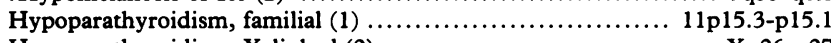

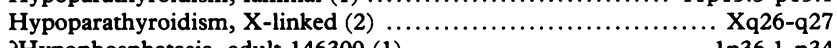

?Hypophosphatasia, adult 146300 (1) ..................... 1p36.1-p34

Hypophosphatasia, infantile 241500 (3) ................... 1p36.1-p34

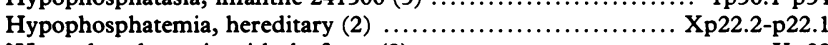

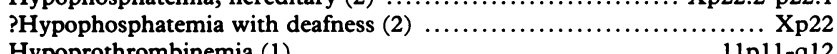

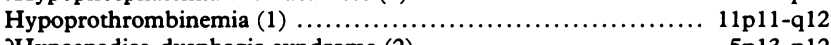

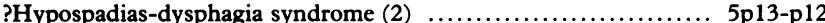

Hypothyroidism, hereditary congenital $(1) \ldots \ldots \ldots \ldots \ldots \ldots \ldots . . .6 \%$ 8q24.2-q24.3

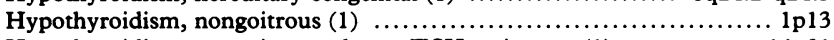

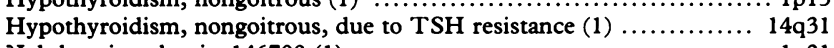

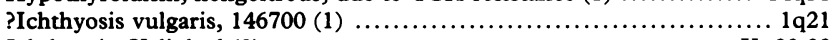

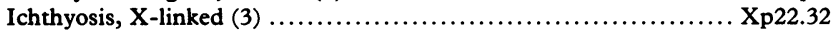

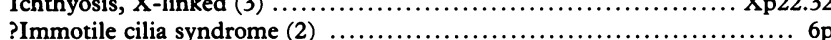

Immunodeficiency, X-linked, with hyper-IgM (2) .............. Xq24-q27

Incontinentia pigmenti, familial (2) ........................ Xq27-q28

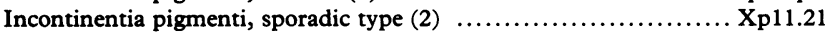

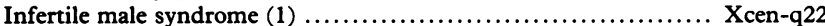

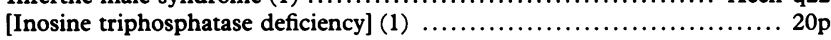

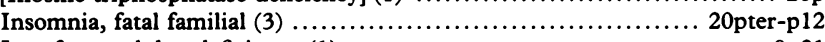

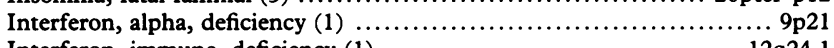

Interferon, immune, deficiency (1)....

? Isolated growth hormone deficiency

due to defect in GHRF (1) .......................20p11.23-qter

Isolated growth hormone deficiency, Illig type with absent $\mathrm{GH}$ and

Kowarski type with bioinactive GH (3) ................. 17q22-q24

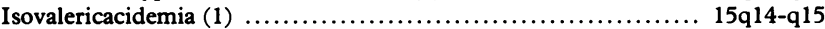

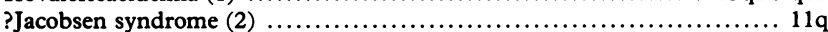

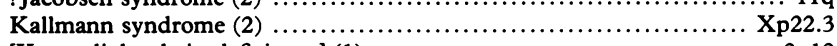

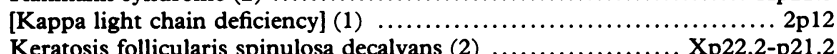

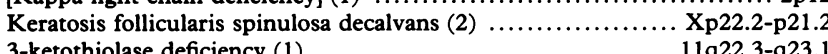

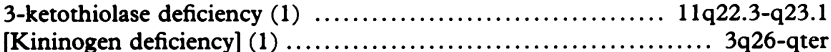

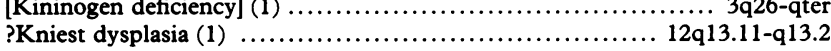

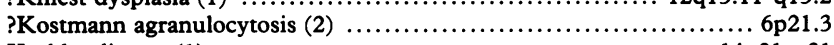

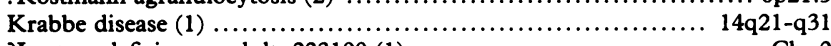

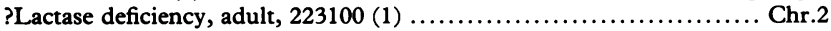

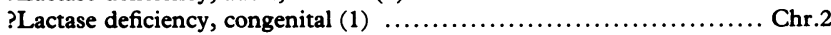

?Lactoferrin-deficient neutrophils, 245480 (1) ................. 3q21-q23

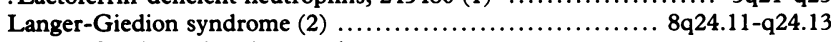

Langer-Saldino achondrogenesis-

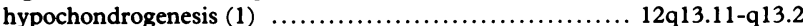

Laron dwarfism (1) ..................................... 5p13-p12

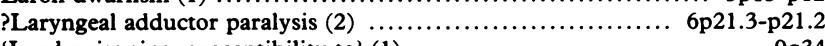

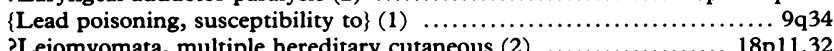

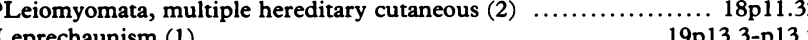

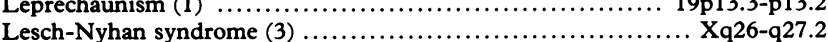

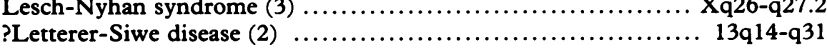

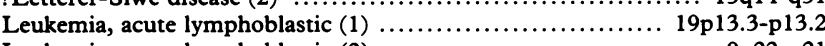

Leukemia, acute lymphoblastic (2) .......................... 9p22-p21

?Leukemia, acute lymphocytic, with $4 / 11$ translocation (3) .......... 4q21

Leukemia, acute myeloid (2) ............................... 21q22

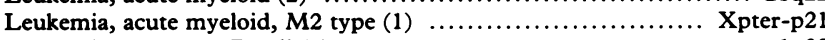

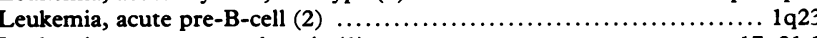

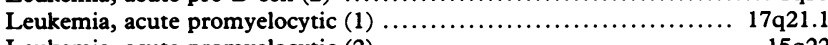

Leukemia, acute promyelocytic (2) ............................ 15q22

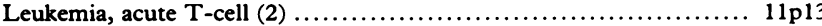

Leukemia, chronic myeloid (3) ............................ 22q11.21

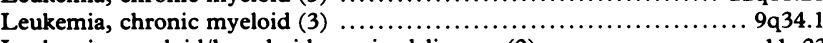

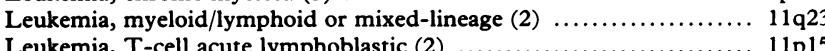


Table 2-contd

Disorder

Leukemia, T-cell acute lymphoblastic (2)

Leukemia, T-cell acute lymphoblastoid (2)

Leukemia, T-cell acute lymphocytic (2)

?Leukemia, transient (2)

Leukemia-1, T-cell acute lymphoblastic (3)

Leukemia-2, T-cell acute lymphoblastic (3)

Leukemia/lymphoma, B-cell, 1 (2)

Leukemia/lymphoma, B-cell, 2 (2)

Leukemia/lymphoma, B-cell, 3 (2)

Leukemia/lymphoma, T-cell (2)

Leukemia/lymphoma, T-cell (2)

Leukemia/lymphoma, T-cell (3)

Leukocyte adhesion deficiency (1) ...

$\mathrm{Li}$-Fraumeni syndrome (1)

Lipoamide dehydrogenase deficiency (1)

Lipoma (2)

Liver cell carcinoma (1)

Long QT syndrome (2)

Lowe syndrome (2)

Lupus erythematosus, systemic, 152700 (1)

Lymphoproliferative syndrome, X-linked (2)

?Lynch cancer family syndrome II (2)

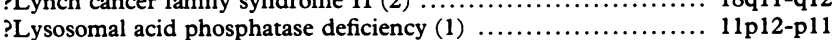

Macrocytic anemia of $5 \mathrm{q}$ - syndrome, refractory $(2) \ldots \ldots \ldots \ldots \ldots, 5 \mathrm{q} 12-\mathrm{q} 32$

Macular dystrophy, atypical vitelliform $(2) \ldots \ldots \ldots \ldots \ldots \ldots \ldots \ldots . \ldots \ldots 24$

Macular dystrophy, North Carolina type (2) ................ 6q13-q21

Macular dystrophy, vitelliform type (2) ...................... 113

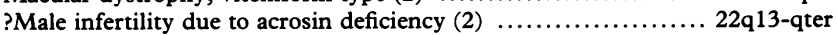

?Male infertility, familial (1) ................................ 11 p13

?Male pseudohermaphroditism due to defective $\mathrm{LH}(1) \ldots \ldots \ldots \ldots 19 \mathrm{q} 13.32$

Malignant hyperthermia, 145600 (3) ........................ 19q13.1

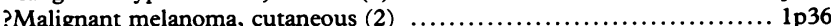

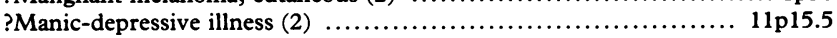

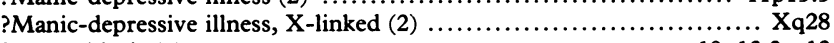

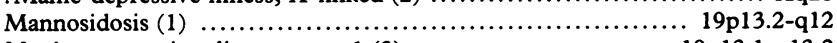

Maple syrup urine disease, type 1 (3) ................... 19q13.1-q13.2

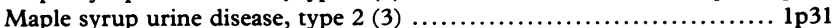

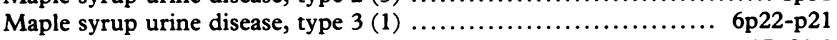

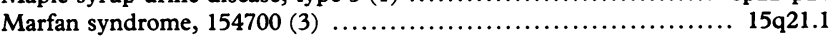

Maroteaux-Lamy syndrome, several forms (1) . .............. 5q11-q13

Martin-Bell syndrome (2) .................................... Xq27.3

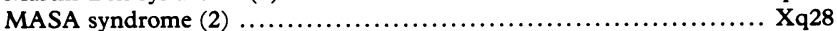

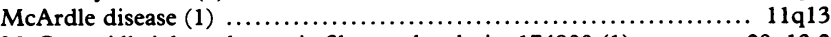

McCune-Albright polyostotic fibrous dysplasia, 174800 (1) ........20q13.2

[McLeod phenotype] (2) ............................... Xp21.2-p21.1

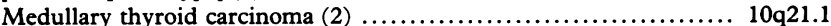

Megacolon (2) ........................................ 13q22.1-q32.1

Megalocornea, X-linked (2) .............................. Xq21.3-q22

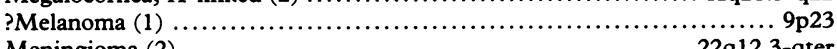

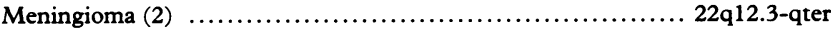

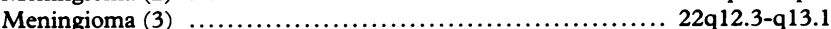

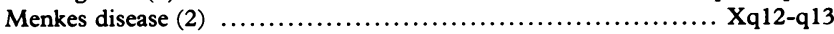

Mental retardation of WAGR (2) $\ldots \ldots \ldots \ldots \ldots \ldots \ldots \ldots \ldots \ldots \ldots \ldots \ldots$
Mental retardation, X-linked, syndromic-1, with dystonic

movements, ataxia, and seizures (2) $\ldots \ldots \ldots \ldots \ldots$

dysmorphism and cerebral atrophy (2) ...............

dysmorphism and cerebral atrophy (2) $\ldots \ldots \ldots \ldots$
Mental retardation, X-linked, syndromic- 3 , with

spastic diplegia (2)

Mental retardation, X-linked, syndromic-4, with

congenital contractures and low fingertip arches (2) ............ Xq13-q22

Mental retardation, X-linked, syndromic-5, with Dandy-Walker

malformation, basal ganglia disease, and seizures (2) ............ Xq25-q27

Mental retardation, X-linked, syndromic-6, with

gynecomastia and obesity (2)

$\mathrm{Xp} 21.1-\mathrm{q} 22$

Mental retardation, X-linked-1, non-dysmorphic (2) ............... Xp22

Mental retardation, X-linked-2, non-dysmorphic (2) ............. Xq11-q12

?Mental retardation, X-linked-3 (2) ............................ Xq28

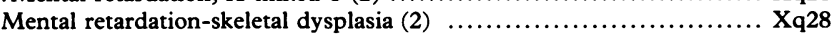

Metachromatic leukodystrophy (1) .................... 22q13.31-qter

Metachromatic leukodystro

due to deficiency of SAP-1 (1)

Methemoglobinemia due to cytochrome b5 deficiency (1) .......... Chr.18

Methemoglobinemia, enzymopathic (1) ................. 22q13.31-qter

Methemoglobinemias, alpha- (1) ..................... 16pter-p13.3

Methemoglobinemias, beta- (1) ......................... $11 \mathrm{p} 15.5$

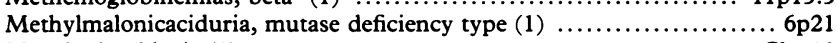

Mevalonicaciduria (1) ...................................... Chr.12

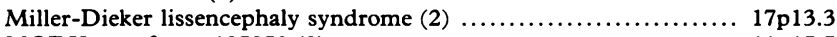

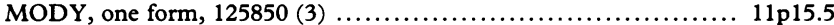

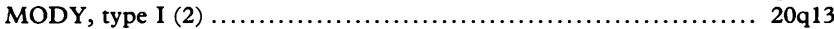

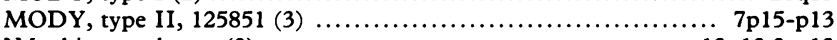

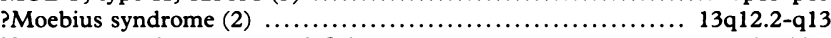

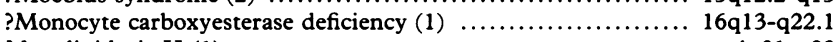

Mucolipidosis II (1)

$4 \mathrm{q} 21-\mathrm{q} 23$

Mucolipidosis III (1)

$4 \mathrm{q} 21-\mathrm{q} 23$

$4 \mathrm{p} 16.3$

Mucopolysaccharidosis I (1)

Mucopolysaccharidosis II (2)

Mucopolysaccharidosis IVB (1)

Mucopolysaccharidosis VII (1)
Disorder

Location

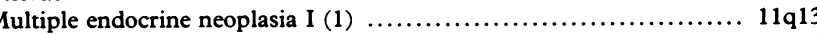

Multiple endocrine neoplasia II (2) .......................... 10q21.1

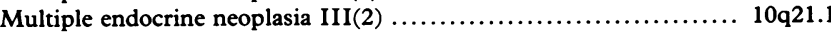

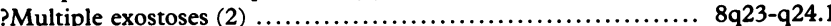

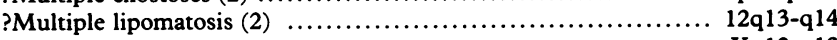

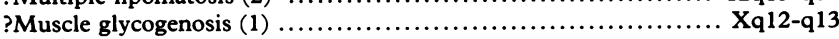

Muscular dystrophy, limb-girdle,

autosomal dominant (2)

$5 q 22.3-q 31.3$

Muscular dystrophy, limb-girdle,

autosomal recessive (2)

$15 \mathrm{q} 15-\mathrm{q} 22$

Myeloperoxidase deficiency (1)...................

7q21.3-q22

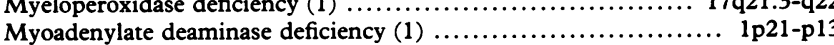

Myoglobinuria/hemolysis due to PGK deficiency (1) ................ Xq13

Myopathy due to CTPase deficiency (1) .................... 1p13-p11

Myopathy due to phosphoglycerate

mutase deficiency (1)

$7 \mathrm{p} 13-\mathrm{p} 12.3$

Myopia-1 (2)

$\mathrm{Xq} 28$

Myotonia congenita, atypical

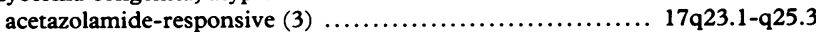

Myotonic dystrophy (2) ................................. 19q13.2-q13.3

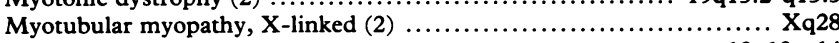

Myxoid liposarcoma (2) ................................. 12q13-q14

?N syndrome, 310465 (1) ............................. Xp22.3-p21.1

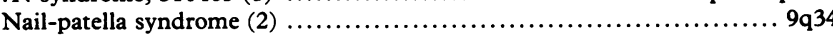

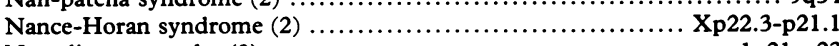

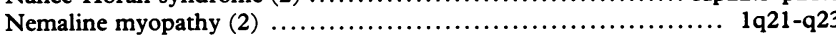

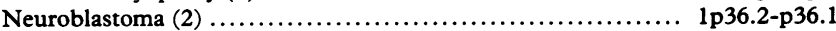

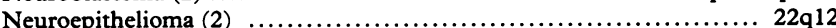

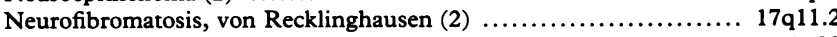

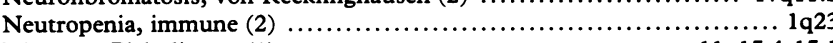

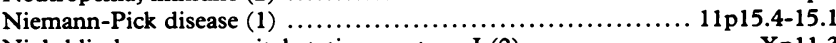

Nightblindness, congenital stationary, type I (2) ................ Xp11.3

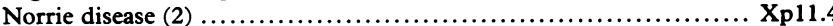

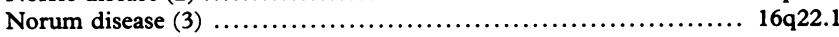

Nucleoside phosphorylase deficiency,

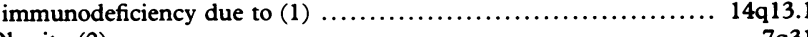

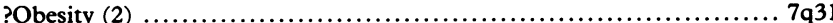

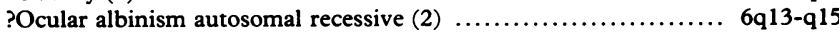

Ocular albinism, Forsius-Eriksson type (2) ..................... Xp11-q11

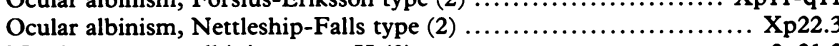

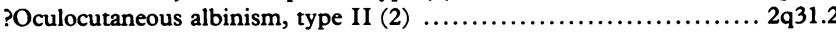

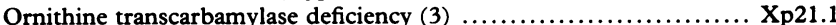

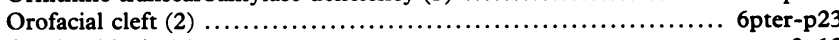

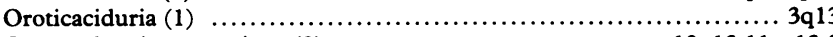

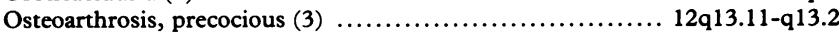

Osteogenesis imperfecta, 2 or more

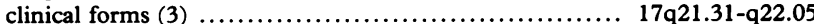

Osteogenesis imperfecta, 2 or more

clinical forms (3)

?Osteopetrosis, 259700 (1)

7q21.3-q22.1

.

Otopalatodigital syndrome, type I (2) ........................... Xq28

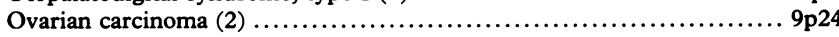

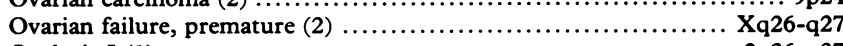

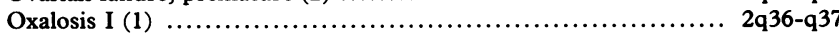

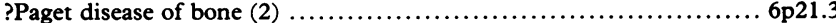

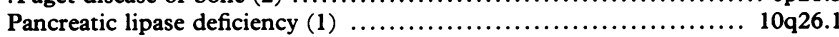

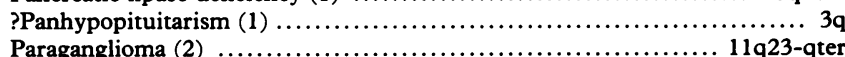

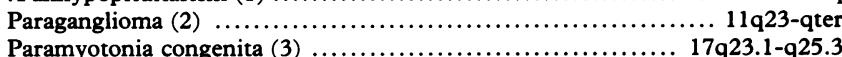

Parathyroid adenomatosis 1 (2) $\ldots \ldots \ldots \ldots \ldots \ldots 13$

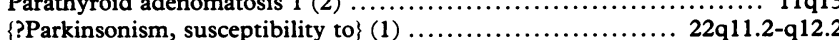

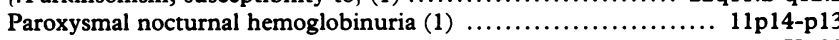

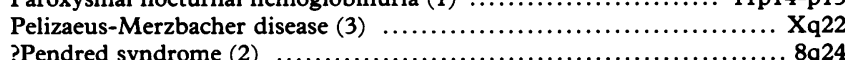

?Pendred syndrome (2)

Periodontitis, juvenile (2) .................... 4q11-q13

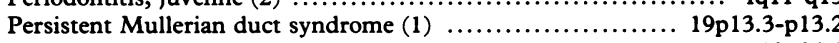

Phenylketonuria (3) ........................

reductase deficiency (1)

Phosphoribosylpyrophosphate

synthetase-related gout (1)

?Phosphorylase kinase deficiency of

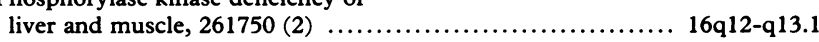

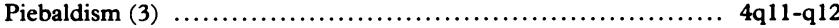

Pituitary tumor, growth-hormone-secreting (1) ................ 20q13.2

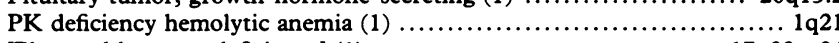

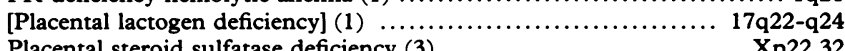

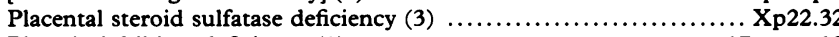

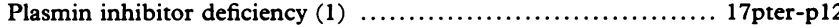

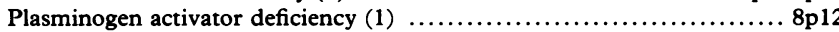

Plasminogen deficiency, types I and II (1) ...................... 6q26-q27

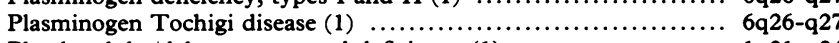

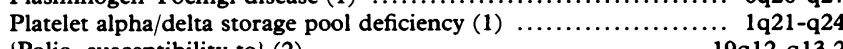

\{Polio, susceptibility to $(2)$

Polycystic kidney disease (2)

$9 \mathrm{q} 12-\mathrm{q} 13.2$

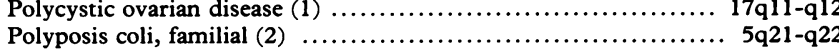

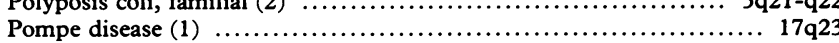

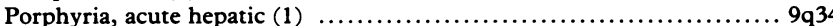


Table 2-contd

Disorder

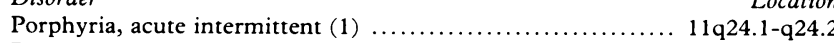

Porphyria, Chester type (2)

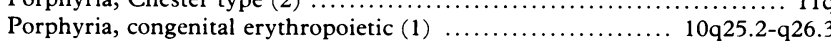

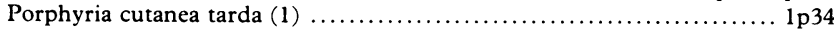

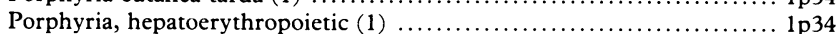

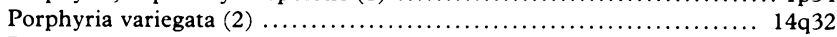

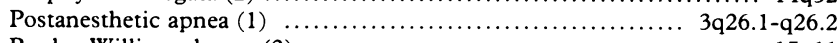

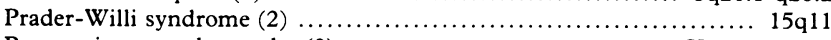

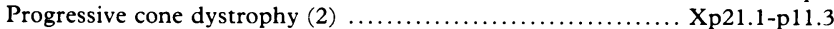

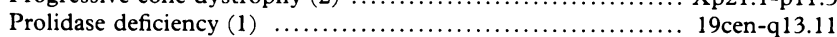

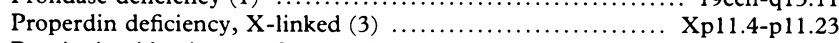

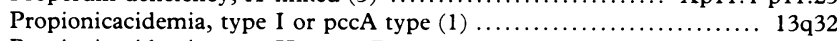

Propionicacidemia, type II or pccB type (1) ................ 3q21-q22

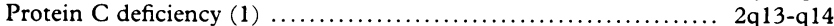

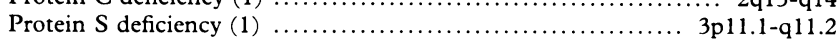

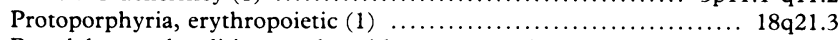

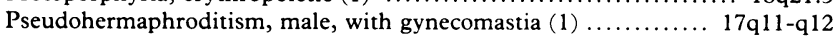

Pseudohypoaldosteronism (1) .............................. 4q 31.1

Pseudohypoparathyroidism, type Ia (1) ....................... 20q13.2

Pseudo-vitamin D dependency rickets 1 (2) ..................... 12q14

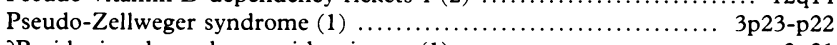

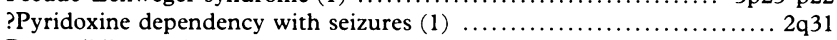

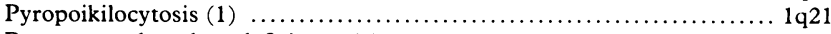

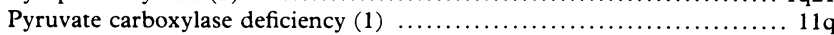

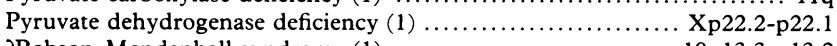

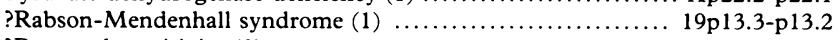

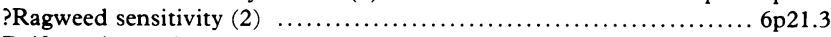

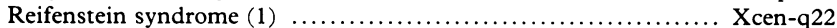

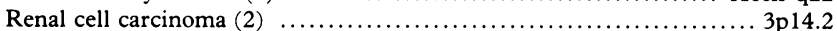

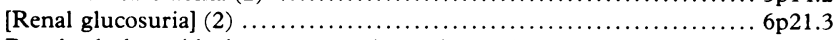

Renal tubular acidosis-osteopetrosis syndrome $(1) \ldots \ldots \ldots \ldots \ldots \ldots \ldots, 8 \mathrm{q} 22$

?Retinal cone dystrophy-1 (2) ........................... 6q25-q26

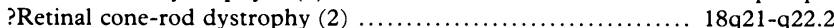

Retinitis pigmentosa, autosomal dominant $(3) \ldots \ldots \ldots \ldots \ldots \ldots \ldots$ 3q21-q24

Retinitis pigmentosa, peripherin-related (3) $\ldots \ldots \ldots \ldots \ldots \ldots \ldots 6,6 \mathrm{p} 21.1$-cen

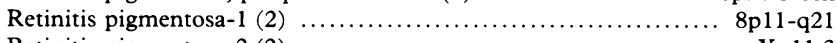

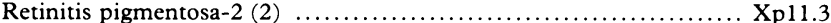

Retinitis pigmentosa-3 (2) ............................ Xp21.3-p21.1

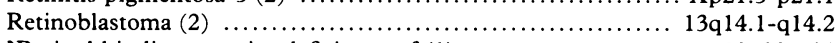

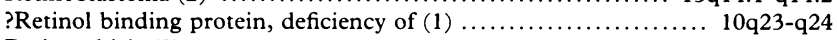

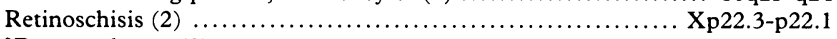

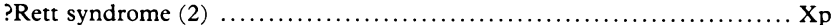

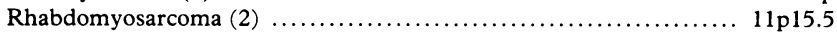

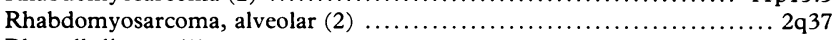

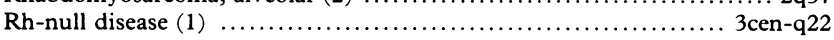

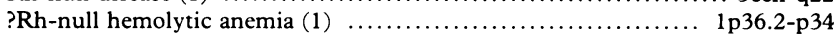

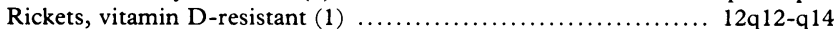

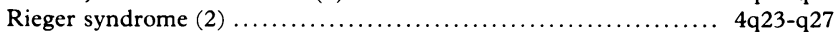

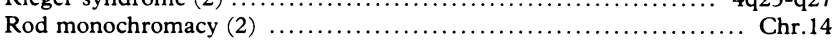

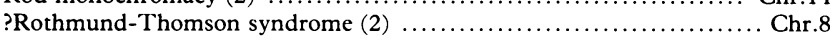

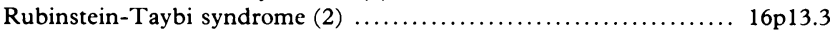

Salivary gland pleomorphic adenoma $(2) \ldots \ldots \ldots \ldots \ldots \ldots \ldots \ldots \ldots, 8 \mathrm{q} 12$

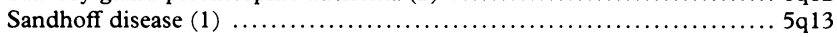

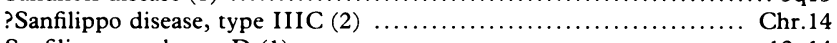

Sanfilippo syndrome D (1) ............................... 12q14

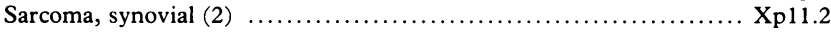

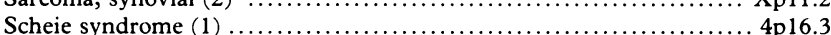

?Schizophrenia (2) ...................................... 5q11.2-q13.3

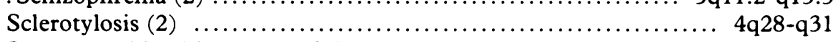

Severe combined immunodeficiency

due to ADA deficiency (1)

$20 \mathrm{q} 13.11$

Severe combined immunodeficiency

due to IL2 deficiency (1)

Severe combined immunodeficiency,

HLA class II-negative type (1)

$4 \mathrm{q} 26-\mathrm{q} 27$

Severe combined

?Sialidosis (2)

Sickle cell anemia (1)

$19 \mathrm{p} 13.1$

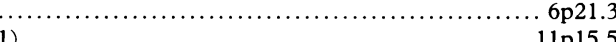

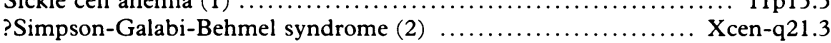

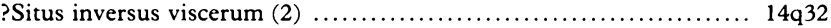

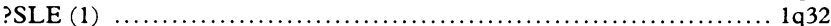

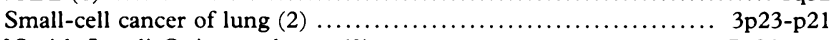

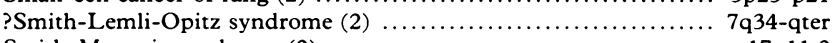

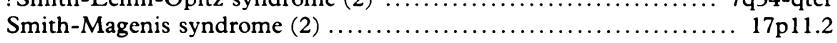

Disorder

Location

Spastic paraplegia, X-linked, uncomplicated (2) .............. Xq21-q22

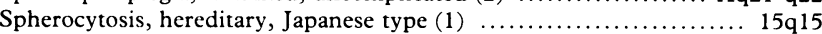

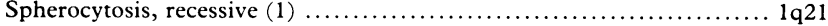

Spherocytosis-1 (3) .................................. 14q22-q23.2

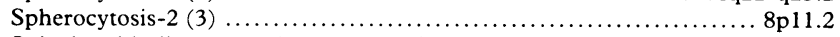

Spinal and bulbar muscular atrophy of

Kennedy, 313200 (3)

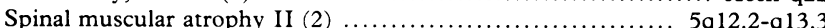

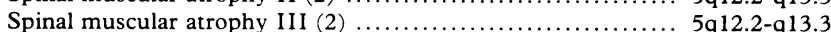

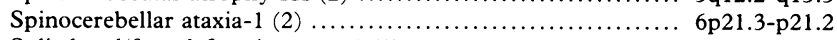

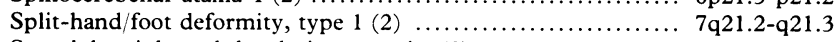

Spondyloepiphyseal dysplasia congenita (3) .............. 12q13.11-q13.2

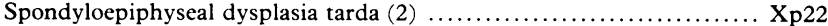

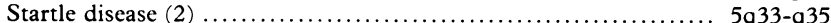

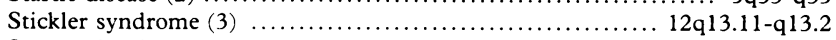

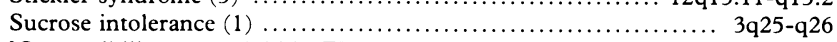

?Susceptibility to amyloid in FMF, 249100 (1) ................. 11 pter-p12

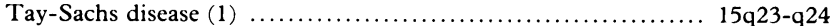

Testicular feminization (1) ................................ Xcen-q22

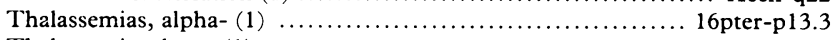

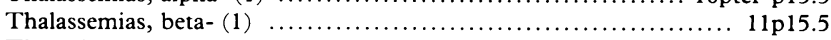

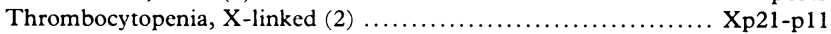

Thrombophilia due to elevated HRG (2) ................... 3p14-qter

Thrombophilia due to excessive

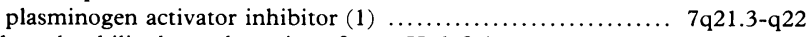

Thrombophilia due to heparin cofactor II deficiency (1) ............ 22q11

Thyroid hormone resistance, 274300,188570 (3) ................. 3p24.3

Thyroid iodine peroxidase deficiency $(1) \ldots \ldots \ldots \ldots \ldots \ldots \ldots \ldots \ldots \ldots$ 2pter-p12

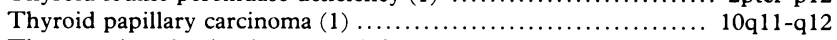

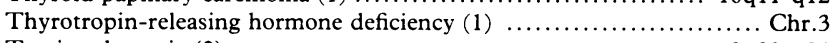

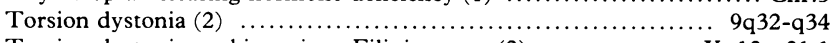

Torsion dystonia-parkinsonism, Filipino type (2) ............ Xq12-q21.1

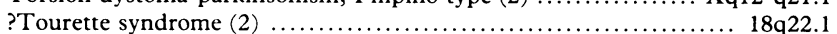

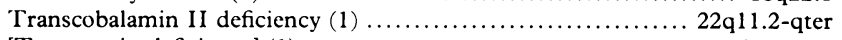

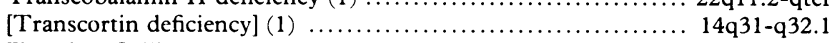

Treacher Collins mandibulofacial dysostosis (2) ........... 5q31.3-q33.3

Trichorhinophalangeal syndrome, type I (2) ................. 8q24.12

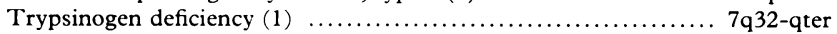

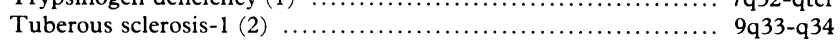

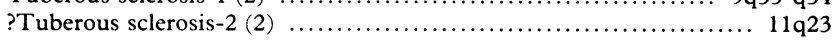

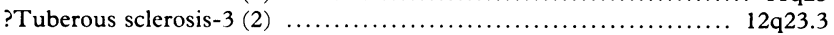

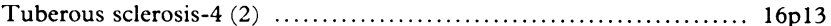

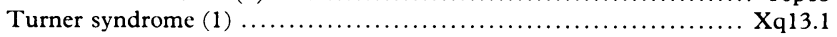

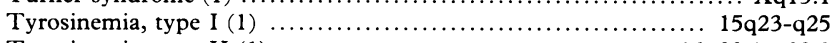

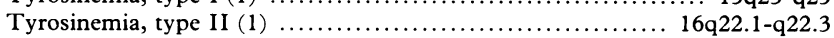

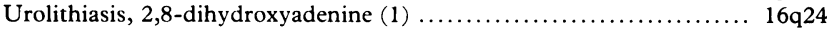

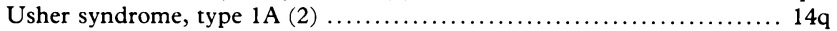

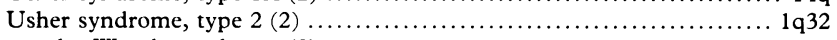

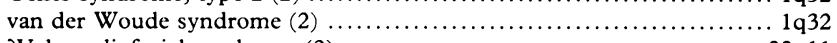

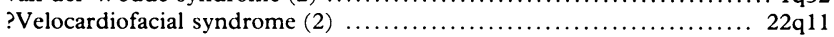

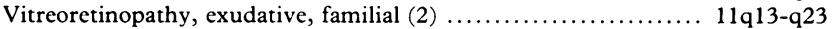

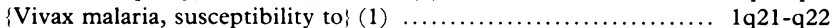

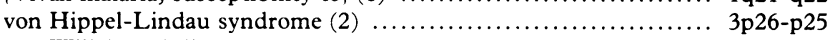

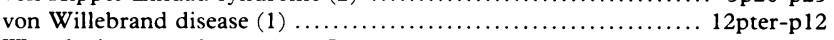

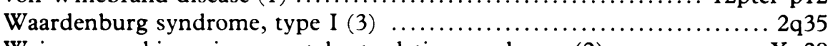

Waisman parkinsonism-mental retardation syndrome (2) ........... Xq28

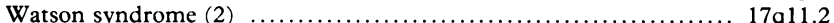

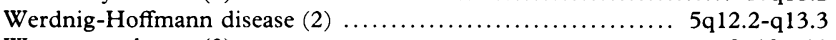

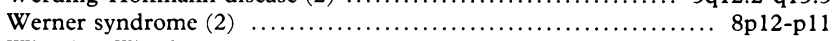

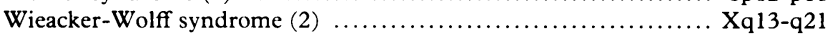

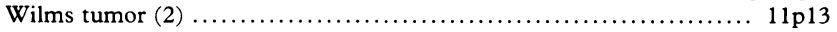

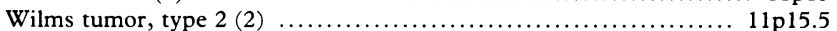

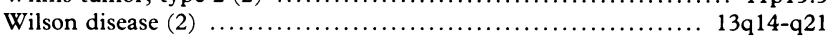

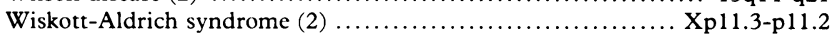

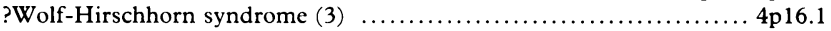

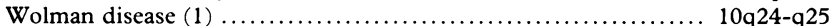

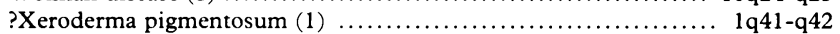

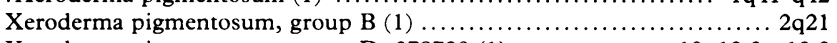

Xeroderma pigmentosum, group D, 278730 (1) ............. 19q13.2-q13.3

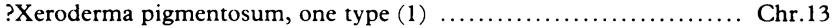

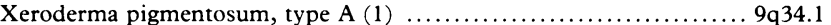

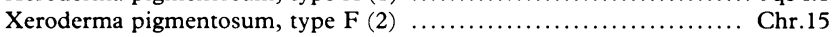

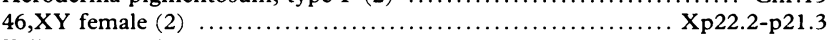

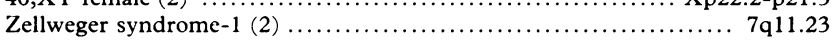

